Florida International University

FIU Digital Commons

FIU Electronic Theses and Dissertations

University Graduate School

$5-28-2019$

\title{
Molecular Mechanism(s) of Zika virus infection and associated neuropathogenesis
}

Chet Raj Ojha

cojha001@fiu.edu

Follow this and additional works at: https://digitalcommons.fiu.edu/etd

Part of the Molecular and Cellular Neuroscience Commons, Nervous System Diseases Commons, and the Virus Diseases Commons

\section{Recommended Citation}

Ojha, Chet Raj, "Molecular Mechanism(s) of Zika virus infection and associated neuropathogenesis" (2019). FIU Electronic Theses and Dissertations. 4269.

https://digitalcommons.fiu.edu/etd/4269

This work is brought to you for free and open access by the University Graduate School at FIU Digital Commons. It has been accepted for inclusion in FIU Electronic Theses and Dissertations by an authorized administrator of FIU Digital Commons. For more information, please contact dcc@fiu.edu. 


\section{FLORIDA INTERNATIONAL UNIVERSITY}

Miami, Florida

\section{MOLECULAR MECHANISM(S) OF ZIKA VIRUS INFECTION AND ASSOCIATED NEUROPATHOGENESIS}

A dissertation submitted in partial fulfillment of

the requirements for the degree of

DOCTOR OF PHILOSOPHY

in

BIOMEDICAL SCIENCES

by

Chet Raj Ojha

2019 
To: Dean Robert Sackstein

College of Medicine

This dissertation, written by Chet Raj Ojha, and entitled Molecular Mechanism(s) of Zika Virus Infection and Associated Neuropathogenesis, having been approved in respect to style and intellectual content, is referred to you for judgment.

We have read this dissertation and recommend that it be approved.

Madhavan Nair

Ajeet Kaushik

Tomas Guilarte

Fatah Kashanchi

Nazira El-Hage, Major Professor

Date of Defense: May 28, 2019

The dissertation of Chet Raj Ojha is approved.

Dean Robert Sackstein

College of Medicine

Andrés G. Gil

Vice President for Research and Economic Development and Dean of the University Graduate School

Florida International University, 2019 


\section{DEDICATION}

This work is dedicated to my late father Bishnu Datt Ojha, who had a dream to see me as a doctor, and to my wonderful family. 


\section{ACKNOWLEDGMENTS}

This dissertation is a result of efforts from many people including but not limited to my major professor, committee members, lab members, department, Ph.D. program, families, and friends. I am eternally indebted to all of them. First and the foremost, I would like to extend deepest and strongest gratitude possible to my major professor Dr. Nazira El-Hage, whose encouragement, guidance and support from the beginning to the end have enabled me to complete my dissertation well ahead of the expected time. Dr. El-Hage opened up doors of opportunity for me by sponsoring me for the presidential fellowship provided by the university graduate school, FIU. Moreover, she was instrumental in my being awarded the dissertation year fellowship. I feel fortunate to have the mentor who understands me, appreciates my limitations and always motivates me in addition to her tremendously effective mentoring. Dr. El-Hage taught me to learn from failure and keep the spirit on and to balance work life and family or social life efficiently. In a nutshell, I don't even have any word to express my highest heartfelt gratitude for Dr. El-Hage.

Next, I would like to thank my dissertation committee members Dr. Madhavan Nair, Dr. Ajeet Kaushik, Dean Tomas Guilarte and Dr. Fatah Kashanchi for their time, support, encouragement and insightful feedback in my dissertation work. I am grateful to Dr. Nair for his help, kindness, and encouragement that he provided as a guardian in the Department of Immunology and Nanomedicine. It was my privilege to get Dr. Kashanchi, a virology expert, as my committee member and I will always be indebted for his support, guidance, and mentorship. I also like to thank Dr. Kaushik for always being on my side, for insightful suggestions, and for your friendly support. I was incredibly lucky to have in 
my committee the prominent neuroscientist Dean Guilarte, who provided critical suggestions and encouraged me to work hard.

For the past four years, Dr. El-Hage laboratory was my second home, and I will never forget the part of my life spent with the incredibly supportive team members of the laboratory; Dr. Myosotys Rodriguez, Dr. Mohan Kumar, Dr. Jessica Lapierre, and Hary Estrada Bueno. I am enormously grateful to Dr. Myosotys Rodriguez for her constant support, mentorship, and being a great friend whom I could share, complain and unload my stresses. I am equally grateful to Dr. Mohan Kumar for being with me and supporting me in addition to teaching me animal research. I must appreciate Dr. Jessica Lapierre for being a hard-working immediate senior, which made my life more comfortable in the lab as well as in the Ph.D. program. I would like to thank all professors, post-docs, staffs, and students of the department for your support, collaboration, and kindness.

Additionally, I would like to thank my Ph.D. program director Dr. Alexander Agoulnik and senior program coordinator Ms. Odalys De La Rosa for their tireless support, assistance, and management. I also would like to thank my friend Rajib Dutta and fellow Ph.D. students for your friendship and encouraging support.

Last but not least, I am extremely grateful to my family here and back in Nepal for their love, care, and support, without what I could not make my dream come true. Especially, I would like to thank my mother Ganga Devi Ojha and wife Laxmi Paudyal for their unwavering support and inspiration. Thanks to my kids Diya and Deepit for making me happy and inspiring me to work hard. 


\begin{abstract}
OF THE DISSERTATION
MOLECULAR MECHANISM(S) OF ZIKA VIRUS INFECTION AND ASSOCIATED

NEUROPATHOGENESIS
\end{abstract}

by

Chet Raj Ojha

Florida International University

Miami, Florida

Professor Nazira El-Hage, Major Professor

Zika virus (ZIKV), a mosquito-borne flavivirus, is known to induce various neurodevelopmental disorders including microcephaly and growth retardation in newborns from infected mothers. However, the exact mechanism of ZIKV-associated neurodevelopmental disorders is still unknown. The study was aimed at identifying the molecular mechanism(s) of ZIKV infection using in-vitro and in vivo methods. Using three isolated strains of ZIKV (MR766, R103451, and PRVABC59), we show that the Asian strains of ZIKV are more infective and toxic to glial cells and neurons compared to the African strain. Infection by PRVABC59 induces markedly higher release of inflammatory molecules; IP10, RANTES, IL-6, and IFN- $\beta$. ZIKV cellular entry in glial cells is primarily mediated by Axl receptor irrespective of the viral strains. We show that ZIKV induces unfolded protein response (UPR), toll-like receptor (TLR) and autophagy pathways in the glial cells. Exploring the role of these pathways in ZIKV infection, we report that pharmacological and genetic inhibition of TLR3 causes a marked decrease in ZIKV titers 
and the viral-induced inflammatory response in infected glial cells, while Beclin1, an autophagy protein having a crucial role in the initiation of autophagosome formation, controls the ZIKV-induced host inflammatory responses. We further investigated the role of Beclin1, in the development of neuropathology in-utero using ZIKV-exposed fetus. ZIKV-infected timed-pregnant wild-type C57BL/6J and Beclin1 deficient $\left(\operatorname{Atg} 6^{+-}\right)$mice showed that the Beclin1 protein plays a protective role in both growth and embryonic brain development in the fetus. Mechanistically, deficiency of Beclin1 causes the reduction in microcephaly related genes (MCPH1, ASPM, CASC5, and WDR62) in the fetal brain, which correlate with the overt congenital malformations and growth impairment in fetus and pups born from Atg6 ${ }^{+/-}$mice. Since viral protein (NS1and Env) were detected in pup's brain in the absence of ZIKV genome, we reason that viral proteins transmigrated from the placenta to the fetus by a yet unknown mechanism, probably the secretory autophagy, might be the causal factors for the neurodevelopmental disorder detected in pups born from infected mice. 


\section{TABLE OF CONTENTS}

\section{CHAPTERS}

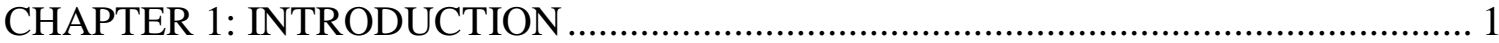

1.1. Introduction of Zika Virus.............................................................................. 1

1.2. Spatiotemporal variants of ZIKV and how they translate to the pathogenesis .... 3

1.3. Mechanism of ZIKV entry into the cells: the role of TAM receptors.................. 5

1.4. Molecular mechanisms potentially involved in the pathology of ZIKV .............. 7

1.5. ZIKV animal research model and challenges ..................................................... 17

1.6. Vaccine and therapy development approach for ZIKV ................................... 17

1.7. Overview of neuron and glial cells .................................................................. 19

1.8. Statement of the problem and objective of the study ......................................... 20

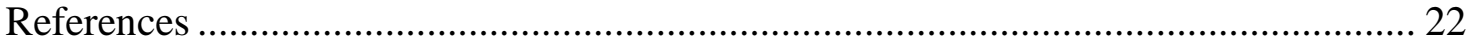

CHAPTER 2: ZIKV INFECTION IN BRAIN CELLS INDUCES INFLAMMATORY

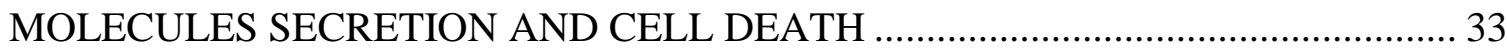

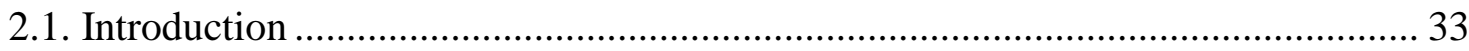

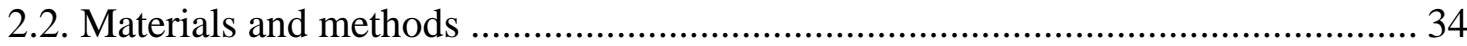

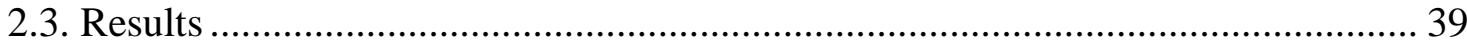

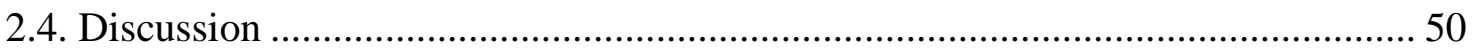

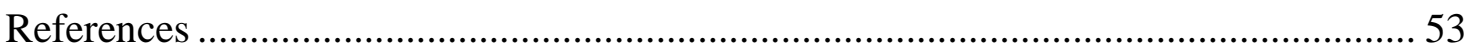

CHAPTER 3. MECHANISM OF ZIKA VIRUS ENTRY INTO HUMAN PRIMARY

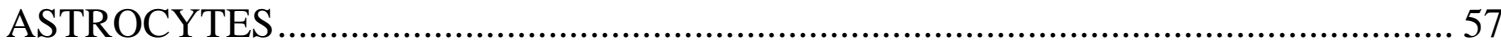

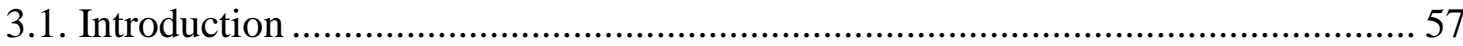

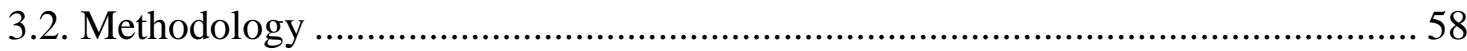

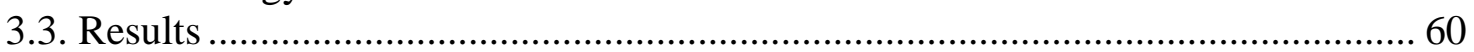

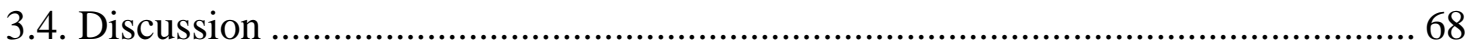

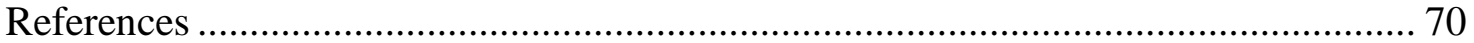

CHAPTER 4: TLR3 REGULATES ZIKV INFECTION AND ASSOCIATED HOST INFLAMMATORY RESPONSES IN HUMAN GLIAL CELLS ................................. 73

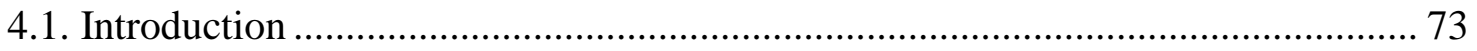

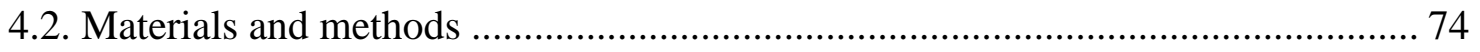

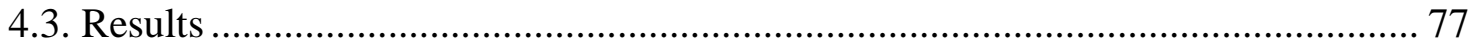

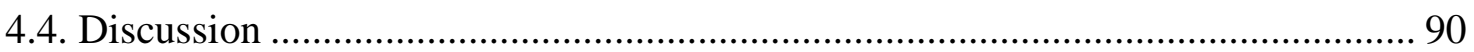

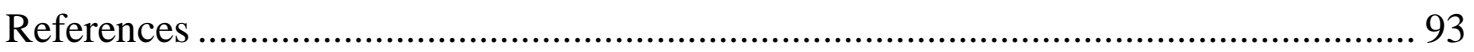

CHAPTER 5: ROLE OF AUTOPHAGY PATHWAY IN ZIKV INFECTION AND

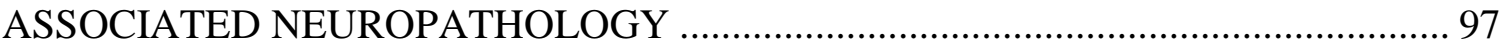

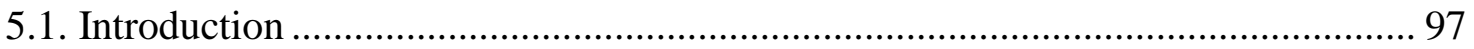

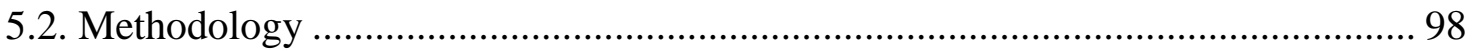

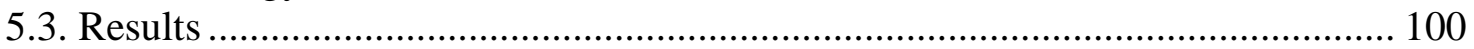

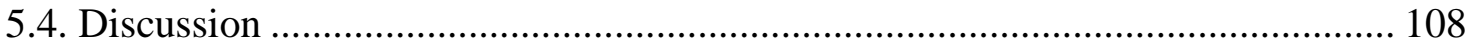

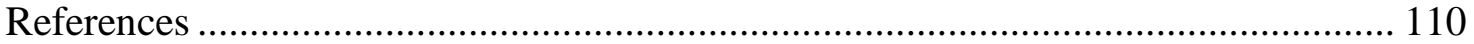




\section{CHAPTER 6. INFECTION WITH ZIKV CAUSES GROWTH AND BRAIN}

ABNORMALITES IN THE PUPS OF AUTOPHAGY-DEFICIENT MICE................ 113

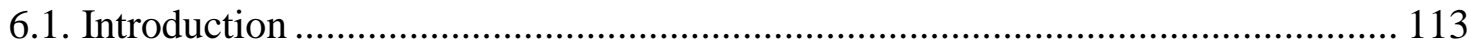

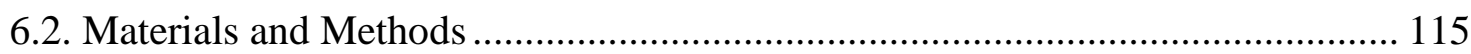

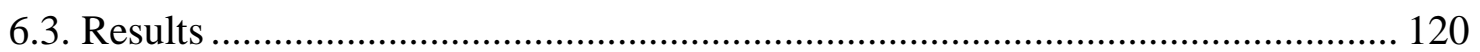

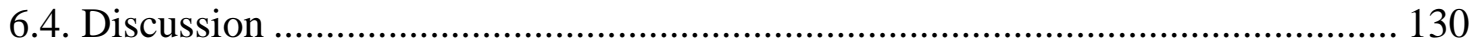

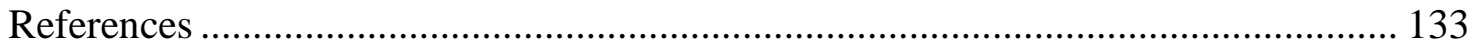

CHAPTER 7. CONCLUSION AND RECOMMENDATIONS ................................ 139

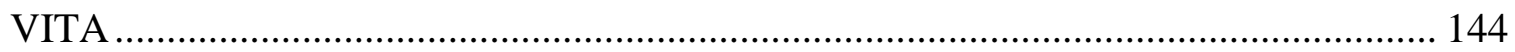




\section{LIST OF FIGURES}

FIGURES

PAGE

Figure 1. Structure and genome of ZIKV ............................................................ 3

Figure 2. Phylogenetic classification showing the potential origin and spread of ZIKV... 4

Figure 3. The major steps in the autophagy pathway. ................................................ 14

Figure 4. Interactions between cells of the central nervous system............................ 20

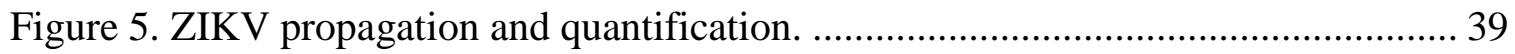

Figure 6. ZIKV infects primary human astrocytes. ............................................... 41

Figure 7. ZIKV replication kinetics and cell viability in primary human astrocytes. ...... 43

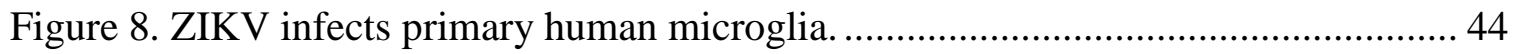

Figure 9. ZIKV infects primary human neuron inducing cell death........................... 45

Figure 10. ZIKV significantly induces secretion of inflammatory molecules in

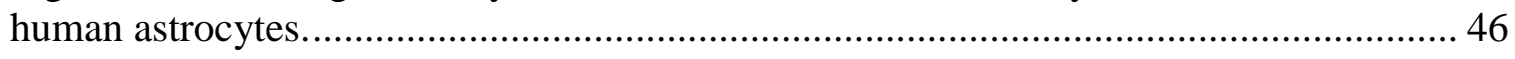

Figure 11. ZIKV induces key antiviral and inflammatory molecules in human

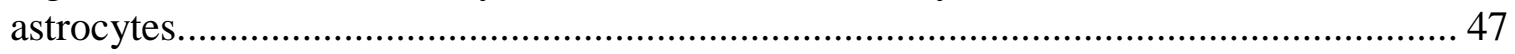

Figure 12. ZIKV induces key antiviral and inflammatory molecules in human microglia.

Figure 13. ZIKV induces minor changes in expression of growth factor in human astrocytes as measured by using Human growth factor antibody array........................... 50

Figure 14. TAM receptors and their ligand Gas6 are expressed in human astrocytes..... 61

Figure 15. Inhibition of Axl by small molecule inhibitor (R428) decreases ZIKV infection in human astrocytes.

Figure 16. Genetic silencing of Axl also decreases ZIKV infection in human

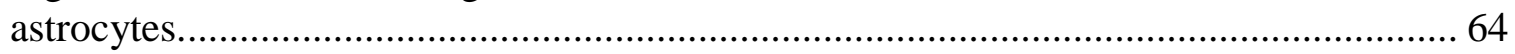

Figure 17. Axl antagonizes IFN response in human astrocytes................................... 65

Figure 18. Inhibition of Tyro3 by small molecule inhibitor (BMS77607) has no effect on ZIKV infection in human astrocytes 
Figure 19. Inhibition of Tyro3 had no additive effect on ZIKV infection in human astrocytes treated with Axl inhibitor.

Figure 20. Axl facilitates ZIKV entry as well as replication within human astrocytes.... 68

Figure 21. ZIKV induces DNA damage and ER stress in human astrocytes. 78

Figure 22. ZIKV induces unfolded protein response in human astrocytes through PERK. 80

Figure 23. Silencing of PERK slightly reduces ZIKV replication in human astrocytes... 81

Figure 24. ZIKV perturbs TLR signaling pathway in human astrocytes. 82

Figure 25. ZIKV induces downstream signaling molecules in the TLR3 signaling pathway; IRF3 and NF-кB...... 84

Figure 26. Silencing of TLR3 reduces ZIKV infection and replication. 86

Figure 27. Silencing of TLR3 reduces ZIKV-induced antiviral and inflammatory molecules secretion 88

Figure 28. TLR3 regulates ZIKV replication and inflammatory response in human microglia.

Figure 29. Silencing of TLR3 reverts ZIKV-induced upregulation of downstream signaling molecules. 90

Figure 30. ZIKV induces autophagosome formation and blocks autophagosomelysosome fusion. 101

Figure 31. ZIKV mediated autophagosome induction and subsequent blockade in autophagosome-lysosome fusion confirmed by the cell-based assay.

Figure 32. Modulation of Autophagy pathway shows no significant changes in viral replication. 103

Figure 33. Modulation of Autophagy pathway shows a minor effect in ZIKV mediated secretion of inflammatory molecules. 104

Figure 34. Silencing of Beclin1 does not affect the replication of ZIKV. 105

Figure 35. Silencing of Beclin1 exacerbates ZIKV-induced inflammatory responses in human astrocytes. 106 
Figure 36. Silencing of TLR3 reverts ZIKV-mediated autophagy induction................ 108

Figure 37. Experimental design for mouse infection model.................................... 117

Figure 38. ZIKV establish productive infection in numerous organs of wild type and Beclin1 deficient pregnant mice....

Figure 39. Infection with Different strains of ZIKV (MR766 and PRVABC59).

Figure 40. Beclin1 deficiency impairs survival and growth in offspring exposed to ZIKV in utero.

Figure 41. Expression of microcephalic genes was decreased in offspring exposed

Figure 42. ZIKV infects mixed mouse glia and induces inflammatory molecules.

Figure 43. ZIKV NS1 and Env proteins cause increased secretion of several inflammatory molecules in $\operatorname{Atg} 6^{+/-}$-derived glia. 


\section{ABBREVIATIONS AND ACRONYMS}

Autophagy-related proteins

ATG

Chikungunya virus

CHIKV

Congenital Zika syndrome

CZS

Dengue virus

DENV

Endoplasmic reticulum

ER

Glial fibrillary acidic proteins

GFAP

Guillain-Barre syndrome

GBS

Interferon

IFN

Interferon alpha and beta receptor subunit 1

IFNAR1

Interferon Gamma induced protein 10

IP10

Interferon regulatory factor

IRF

Japanese encephalitis virus

JEV

Microcephalin

$\mathrm{MCHC}$

Microtubule associated protein 2

MAP2

Microtubule-associated protein light chain-3

LC3

Nuclear factor kappa B

$\mathrm{NF}-\kappa \mathrm{B}$

Protein kinase RNA-like ER kinase

PERK

Sequestosome 1

SQSTM1/p62

Toll-like receptors

TLRs

Tyro3 Axl MER

TAM

Unfolded protein response

UPR 


\section{CHAPTER 1: INTRODUCTION}

\subsection{Introduction of Zika Virus}

Zika virus (ZIKV) is a member of Flavivirus genus and closely related to other clinically important flaviviruses including Dengue virus (DENV), West Nile virus (WNV), Chikungunya virus (CKV) and Japanese encephalitis virus (JEV) [1]. In terms of historical timeline, ZIKV was first isolated in 1947 from monkey host in the Zika forest of Uganda during a yellow fever surveillance program. A few years later in 1952, a neutralizing antibody against ZIKV was isolated from human sera [2, 3]. The first major human outbreak of ZIKV infection was reported in Yap Island of Micronesia in 2007 followed by a major public health concern from outbreaks in French Polynesia in 2013 and most recently in Brazil, where the infection was linked to increased prevalence of Guillain-Barre syndrome (GBS) and microcephaly, respectively [4, 5]. In a case study of French Polynesians diagnosed with GBS, infection by ZIKV was reported in ninety-eight percent (41 out of 42) of patients by the detection of anti-ZIKV-IgM or anti-ZIKV-IgG antibodies. In the study, every individual cases with GBS had neutralizing antibodies against ZIKV, as compared to $56 \%$ detected in the control population [6]. In Colombia, $42 \%$ of people who tested positive for ZIKV were diagnosed with GBS; reflecting the increase in cases of this disease with the ZIKV outbreak in 2015-2017 [7]. In July 2015, the Brazilian department of health announced a direct association between ZIKV and GBS and three months later, ZIKV was reported to be the main cause related to the congenital abnormality detected in microcephalic newborns. A retrospective analysis of ZIKV outbreak in French Polynesia further provided an evidence for maternal ZIKV infection in the first trimester of pregnancy as the risk factor for microcephaly in fetuses [8]. 
A great deal of attention was placed on the spread of and protection against the Aedes mosquitos, as they are the predominant species of mosquitos transmitting the virus. ZIKV primarily is a sylvatic virus transmitted between mosquitos and non-human primates. Aedes albopictus and Aedes aegypti are the most commonly responsible vectors for ZIKV transmission with A. aegypti being associated with the latest outbreak in South America. After the recent outbreaks, it became clear that ZIKV transmission was not limited to the mosquito-borne route. Several cases of human to human ZIKV transmission possibly via sexual contact have already been reported $[9,10]$. Detection of viral particles in breast milk and evidence of viral damage in the placenta of ZIKV infected mother further indicates the possibility of vertical transmission $[11,12]$. Human to human transmission via sexual contact, body fluids, and blood transfusion is the unique feature of ZIKV compared to other flaviviruses and might be the factor enhancing the stability of ZIKV in different body fluids and the broad viral tropism [13].

Similar to other flaviviruses, ZIKV is an enveloped and icosahedral virus having single-stranded positive-sense RNA as a genome. The RNA is about $11 \mathrm{~kb}$ long and contains a single open reading frame (ORF) flanked by noncoding regions on both 5' and 3' sides $[14,15]$. The ORF encodes a polyprotein of 3419 amino acids that is cleaved by both host and viral proteases post-translationally into the capsid (C), precursor of the membrane (prM), envelope (E) and 7 nonstructural proteins (NS1, NS2A, NS2B, NS3, NS4A NS4B, and NS5) (Figure 1) [16]. In addition to controlling viral transcription and replication, the NS proteins are involved in attenuating host antiviral responses [17-19], while the envelope (E) protein mediates cellular attachment, entry, and fusion and is the primary target for neutralizing antibodies [20, 21]. 


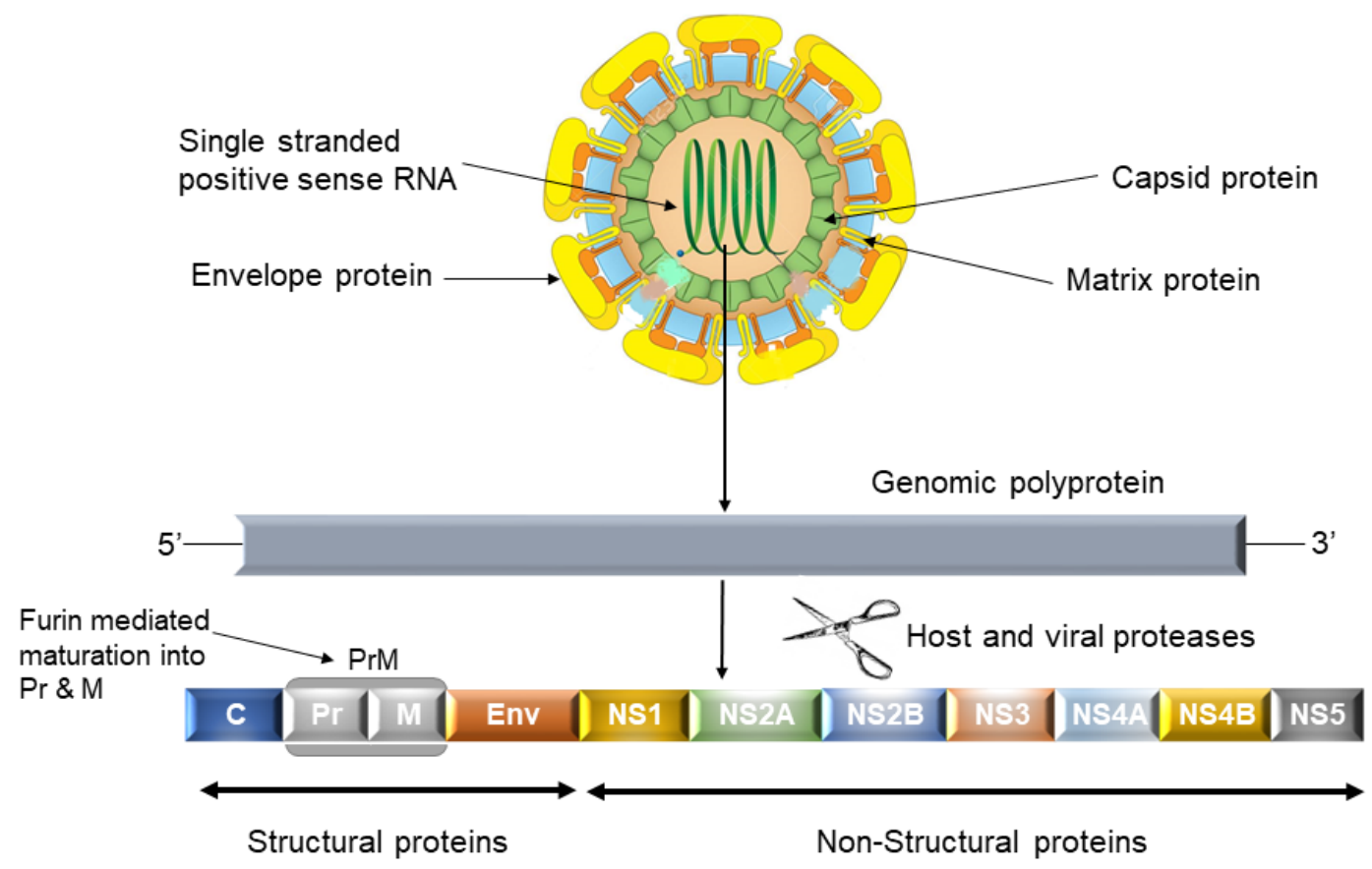

Figure 1. Structure and genome of ZIKV.

\subsection{Spatiotemporal variants of ZIKV and how they translate to the pathogenesis}

Genetic and phylogenetic studies have revealed that ZIKV has evolved from 3 distinct lineages including the West African (Nigerian cluster), the East African (MR766 prototype cluster), and the Asian lineage (Figure 2). Genetic studies on the NS5 gene indicate that ZIKV was most likely originated from East Africa and spread towards West Africa and Asia approximately 50-100 years ago [22, 23]. The African lineage viruses were sporadically associated with human infections over the past century whereas the Asian lineages have emerged as a new public health burden because of their capacity of transmitting from human to human and causing neurological abnormalities [24]. Genetic changes among the ZIKV lineages are attributed to the global spread of new phenotypes and emergence of increased neuropathogenicity. Phylogenetic and amino acid variant analysis of the spatiotemporally different strains of ZIKV; MR766 (Uganda 1947), 
FSS13025 (Cambodia 2010), P6-740 (Malaysia 1966), PRVABC59 (Puerto Rico 2015), and DAKAR 41519 (Senegal 1984); revealed their distinct lineages and significant amino acid differences in the viral polyprotein. Higher levels of accumulated changes were reported in the envelope protein, the terminal region of the prM, the NS2A and the NS5 protein regions of ZIKV genomes [24].

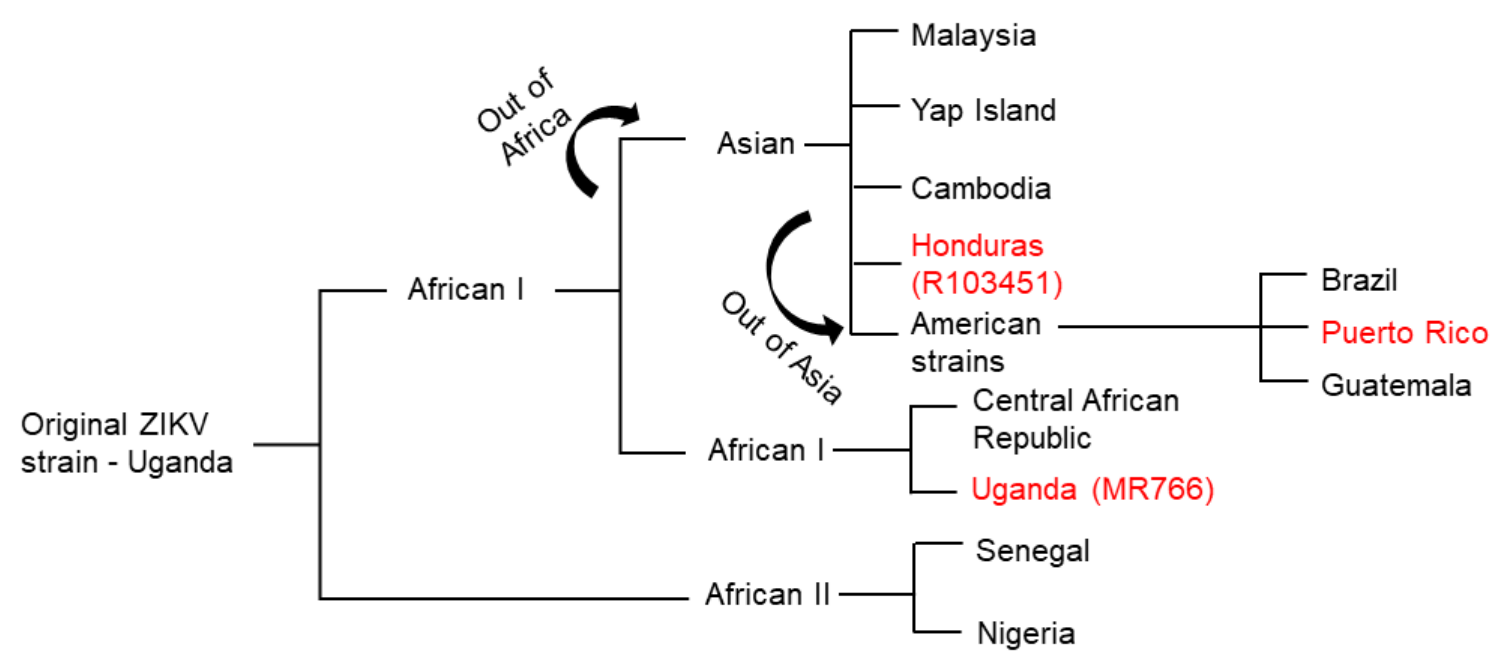

Figure 2. Phylogenetic classification showing the potential origin and spread of ZIKV.

Lineage-specific differences in ZIKV infectivity, virulence and pathologies have been reported using in vivo mouse and in vitro cell culture model system. Studies in which either the signal transducer and activator of transcription-2 knock out (Stat $2^{--1}$ ) or the interferon alpha receptor 1 knock out $\left(\right.$ Ifnar $\left.1^{--}\right)$mouse model were used, showed that the Asian strains including the Puerto-Rican, Malaysian and Cambodian strains of ZIKV caused delayed onset of disease, less severe disease phenotypes and lower lethality rates when compared to the African strains (MR766) of ZIKV [24]. However, others have reported that both the Asian strain (H/PF/2013) and the African strain (Dakar 41519) had similar virulence and caused $100 \%$ lethality in the Ifnar $^{-/-}$mouse model in a different study [25]. The French Polynesian strain (H/PF/2013) is reported to be more lethal compared to 
the other viral strains within the Asian lineage of ZIKV. The inconsistency between the studies in regards to the Asian strains might be related to the inherent differences within the specific lineages, differences in their amino acid sequences and the study design including the viral inoculation dose. Nonetheless, these studies have revealed the differences in pathogenesis and lethality associated with spatiotemporally different ZIKV lineages or strains.

In vitro studies have reported a higher infection rate in neural stem cells (NSCs) and astrocytes with the African lineage ArB41644 as compared to the Asian lineage PRVABC59 and H/PF/2013. Also, NSCs infected with ArB41644 showed higher levels of cell cycle impairment and antiviral response when compared to cells infected with PRVABC59 and H/PF/2013. The same study also reported a significant up-regulation in the genes encoding for DExD/H-Box helicase 58 (DDX58), interferon induced with helicase C domain 1 (IFIH1), toll-like receptor-3 (TLR3), and interferon beta 1 (IFNB1) and downregulation of C-X-C motif chemokine ligand 8 (CXCL8) in NSCs infected with African strains, while significant down-regulation in the genes encoding for the C-X-C Motif chemokine ligand 10 (CXCL10), caspase 1 (CASP1) and cathepsin S (CTSS) was reported in NSCs infected with Asian strains [26]. These findings further suggest that the molecular mechanism underlying ZIKV infection and pathogenesis might be lineagespecific and a single therapeutic approach targeting the African lineage may not necessarily be effective against the Asian lineage of ZIKV.

\subsection{Mechanism of ZIKV entry into the cells: the role of TAM receptors}

Similar to other members of the Flavivirus, ZIKV enters the cell presumably by receptor-mediated endocytosis $[27,28]$. However, the molecular mechanisms of viral entry 
into brain cells and the associated pathologies are still poorly understood. Current studies suggest that Tyro3 Axl MER (TAM) family of receptors are shown to be the primary entry factors for ZIKV in various cell types [28, 29]. TAM is a subfamily of receptor tyrosine kinase (RTK) having distinctive domain structure and a unique $\mathrm{KW}(\mathrm{I} / \mathrm{L}) \mathrm{A}(\mathrm{I} / \mathrm{L}) \mathrm{ES}$ sequence in their catalytic domain. They contain two immunoglobulins like ectodomains, two fibrinolectin type III domains, a hydrophilic transmembrane domain, and the intracellular tyrosine kinase domain. The receptor has two traditional ligands; the growth arrest-specific-6 (Gas6) and the protein S [30]. Gas6 can activate all three receptors in the order of Axl> Tyro3>> MERTK, while protein $\mathrm{S}$ can activate tyro3 and MERTK. The tyrosine kinase activity of the TAM receptors has an important role in controlling excessive inflammation by dampening the innate immune response in cells, in promoting tissue repairs and in clearance of apoptotic cells [31].

Out of the three TAM receptors, Axl is the most studied receptor in regards to ZIKV infection and was originally cloned from cancer cells [32]. Axl is involved in the innate immune response as well as other cellular mechanisms including proliferation, migration, and aggregation in different cell types $[33,34]$. Exposure to neutralizing antibody against Axl or by gene silencing using small interfering RNA targeting the Axl gene led to a decrease in viral replication and infection, suggesting a role of Axl as a putative ZIKV entry receptor in human fibroblast cells [35]. Human microglia and astrocytes isolated from developing brain express Axl receptors. Inhibition of Axl by synthetic decoy receptor (MYD1) and Axl kinase inhibitor (R428) have been shown to inhibit ZIKV infection in the glial cells [28]. 


\subsection{Molecular mechanisms potentially involved in the pathology of ZIKV}

Once the virus has made its way inside the host, an integrated defense network comprising of innate and adaptive immune responses works together to thwart viral infections [36]. Viruses and their components are sensed by the host cell via different pattern recognition receptors (PRR) including the Toll-like receptors (TLRs) and the Retinoic acid-inducible gene (RIG)-1 like receptors (RLRs) to initiate a cellular defense [37]. The interferon (IFN) signaling pathway, unfolded protein response (UPR), DNA repair mechanism, autophagy, and apoptosis are major cellular mechanistic defenses that can suppress viral replication and salvage the infected host cell [35, 38-40]. While it is still unclear how ZIKV elicits its pathogenicity related to neurodegeneration and neurodevelopmental disorders, studies have unraveled several potential avenues that may be involved in ZIKV pathogenesis.

\subsubsection{ZIKV and the Endoplasmic Reticulum (ER) pathways}

The ER provides a membrane platform for the biogenesis of flavivirus replication complexes. Flavivirus infection leads to rearrangement of the ER membrane to form an organelle-like structure which provides an environment for viral replication. Viral proteins including structural and nonstructural proteins accumulated in the ER membrane facilitate the rearrangement by changing protein and lipid content of the ER membrane $[41,42]$. The hydrophilic transmembrane viral proteins, NS4A and NS4B, are primarily involved in membrane remodeling $[43,44]$. Membrane remodeling results in the formation of the socalled vesicle pockets or double membrane vesicles (DMVs) that facilitates the assembly of newly synthesized viral components and complete the virion packaging before 
trafficking to the Golgi complex. Therefore, interactions between the ER and flavivirus play a key role in providing a replication platform and assembly site [45].

On the other hand, synthesis of excessive viral protein may lead to ER stress. The intracellular signaling pathway referred to as the unfolded protein response (UPR) pathway next comes into the scene to alleviate the ER stress. The UPR pathway is a homeostatic signaling pathway that is activated to counteract ER stress by transcriptional induction of genes, retardation of global protein synthesis and ER-associated degradation [46, 47]. UPR is induced via either of three arms or stress sensors; protein kinase RNA-like ER kinase (PERK), inositol-requiring protein $1 \alpha(\operatorname{IRE} 1 \alpha)$ and activating transcription factor 6 (ATF6) [46]. UPR mitigates unfolded protein load by pro-survival mechanisms such as ER membrane expansion, decreased influx of proteins into ER, induction of transcription of key components of protein folding. If stress is not under control, then UPR induces selective autophagic degradation of ER (ER-phagy) or apoptosis [46]. ZIKV has been reported to induce the UPR pathway (as shown by upregulation of UPR related genes expression) in human fetal brain cortex, mouse embryonic brain and in vitro hNSCs. Interestingly, a pharmacological inhibitor of PERK (GSK2656157) reverted ZIKV-mediated impairment of neurogenesis and microcephaly-like syndromes in mouse embryos. Similarly, ZIKV associated neurogenic defects were rescued by inhibition of PERK, whereas ZIKV replication was reduced by IRE-1 $\alpha$ inhibitor in cortical cells [48].

ER Stress can also lead to the formation of stress granules (SGs) by phosphorylation of the eukaryotic initiation factor $2 \alpha$ (eIF2 $\alpha$ ). SGs are dynamic cytoplasmic granules composed of cellular mRNAs and stalled pre-initiation complexes. SGs play an important 
role in maintaining RNA homeostasis under stress conditions [49]. SGs restrict viral replication by hiding cellular translational machinery. However, some viruses can counteract the defense mechanism provided by SGs assembly $[50,51]$. In fact, ZIKV has evolved a similar strategy to suppress SGs assembly despite activating UPR and phosphorylating eIF2 $\alpha$ leading to a global translational arrest. ZIKV proteins NS3 and NS4A are involved in the translational arrest, whereas, capsid, NS3, NS2B, and NS4A cooperatively suppress SGs formation $[52,53]$. However, the precise mechanism of ZIKVinduced suppression of SGs formation is still unclear.

\subsubsection{ZIKV pathogenesis and host immune response}

Before the recent outbreaks, ZIKV infection was considered an asymptomatic or mild self-limiting febrile illness resolving within a few days. A wide range of tissue tropism, multiple routes of transmission and association with severe disease involving multi-organs are reported in the recent ZIKV outbreaks [54]. Though ZIKV is thought to be less neurotropic than other neurotropic flaviviruses, the alarming consequence of ZIKV is its tropism for brain cells, particularly neural progenitor cells leading to impairment of brain growth that may result in microcephaly and other neurological disorders [54]. ZIKV is capable of infecting almost all cell types in brain or CNS including astrocytes, microglia $[28,55]$, brain microvascular endothelial cells [56], pericytes [57], oligodendrocytes and neurons [58]. ZIKV can also infect the eye including the cornea and the optic nerves causing uveitis and even blindness [59].

$\mathrm{ZIKV}$ is reported to modulate both the innate and the adaptive immune-branches of the host defense mechanism; however, both branches may not be required to prevent the disease [60]. The innate immune response is the primary host response that controls ZIKV 
infection and related pathogenesis. Type 1 IFN which includes the isoforms of IFN- $\alpha$, IFN$\beta$ and other minor classes (such as IFN- $\varepsilon$, IFN-k, IFN- $\omega$ ) are crucial for antiviral response and are produced by almost all cells in the body [61]. The expression of type I IFN is regulated by an intracellular signaling pathway triggered by recognition of specific viral components such as viral double-stranded (ds) or single-stranded (ss) RNA or the replication intermediate of viral RNA by germline-encoded PRRs [37]. ZIKV induces TLRs (mainly TLR3), RIG-1, melanoma differentiation associated protein 5 (MDA5), the interferon-stimulated genes (ISGs) such as 2'-5' oligoadenylate synthetase 2 (OAS2), ISG15, and Mx dynamin-like GTPase-1 (MX1) along with IFN- $\beta$ in various cell type [35]. The primary role of the innate immune response in controlling ZIKV infection is further supported by the finding that immuno-compromised individuals or animal models are more susceptible to ZIKV infection as well as the progression to overt disease [62]. Ifnar $1^{-/-}$mice are susceptible to ZIKV infection up to six months of age and even the younger mice within the age of 3 weeks can succumb to overt illness [63].

However, in type 1 IFN deficient animal models, the adaptive immune response becomes critical $[60,62]$. ZIKV specific antibodies are reported to play a crucial role in controlling viral replication in the mouse model. Anti-ZIKV antibodies with variable neutralizing potency have been reported from the ZIKV infected patients. Interestingly, high neutralizing responses to ZIKV were associated with pre-existing DENV reactivity, suggesting possible cross neutralization among the members of flavivirus [64]. The envelope (E), pre-membrane (prM) and NS1 proteins are the major targets for ZIKV specific antibodies and therefore, are the attractive components for DNA vaccine [65]. ZIKV-induced T-cell response was studied in immunocompetent C57BL/6 mice by 
tracking surrogate markers expressed by these cells. ZIKV sensitized $\mathrm{CD}^{+}{ }^{+} \mathrm{T}$ cells polarized to a Th1 phenotype while $\mathrm{CD}^{+}{ }^{+} \mathrm{T}$-cells differentiated into the activated effector phenotype, leading to the production of cytokines and cytolytic molecules [66]. This novel ZIKV specific $\mathrm{CD}^{+} \mathrm{T}$ cell epitope identified for the envelope protein and recognized by many cells can pave the way for the design of tetramers to study epitope-specific $\mathrm{T}$ cell responses and development of ZIKV vaccine strategies [66]. Depletions of T cells resulted in a decrease in body weight in ZIKV infected mice [67]. Interestingly, the pregnant C57BL/6 mice are reported to be more responsive to ZIKV-induced diminutions of cellmediated immunity, leading to more viral replication and possible spread of the virus from mother to fetus [67].

For ZIKV to evade and antagonize the host immune responses, the virus may have evolved multiple mechanisms. The nonstructural (NS) proteins, for example, can act as an immune response antagonist. First, ZIKV NS5 protein targets the proteasomal degradation of STAT2, leading to inhibition of type I IFN signaling [68]. The viral proteins, NS1 and NS4B, inhibit RLR-induced IFN- $\beta$ activation at the Tank-binding kinase 1 (TBK1) level, a multifunctional protein implicated not only in the innate immunity but also in apoptosis and cell proliferation. Activations of STAT6 transcription factor and interferon regulatory factor (IRF)-3 are mediated by TBK1 [60]. ZIKV infection disrupts TBK1 relocation from the centrosome to the mitochondria which may lead to impaired mitosis and cell division [69], suggesting TBK1 is one of the major targets of ZIKV infection [60]. The other viral protein NS2B in conjunction with NS3 promotes the degradation of Janus kinase (JAK1) and the inhibition of the JAK-STAT signaling, followed by inhibition of viral-induced apoptosis and increased viral replication. Finally, NS1 and NS4B inhibit the expression of 
Type 1 IFN and consequently the degradation of NS2B and NS3 by the autophagy pathway with subsequent suppression of viral replication by this pathway. These findings suggest that the non-structural proteins act synergistically to restrict host antiviral responses and facilitate viral replication [70].

\subsubsection{ZIKV and the Toll-Like Receptor-3 (TLR-3) signaling pathway}

The TLR3 signaling pathway is a part of the innate immune response and creates an antiviral state in the viral-infected cell. Normally, activation of TLR3 leads to downstream signaling pathway which involves the recruitment of TIR domain-containing adaptor inducing IFN- $\beta$ (TRIF) and subsequent activation of transcription factors such as interferon regulatory factors (IRF)-3, IRF7 and nuclear factor kappa B (NF-kB) ultimately inducing the production of type 1 and type 2 IFN, pro-inflammatory cytokines, chemokines and ISGs $[71,72]$. Though the role of TLR3 in ZIKV pathogenesis is still under investigation, findings with DENV and WNV are not consistent either [73-75]. TLR3 deficient mice $\left(\mathrm{TLR}^{-/-}\right.$) are reported to be resistant to infection with the WNV [73] and the influenza virus [76]. Similarly, TLR $^{-/-}$mice infected with WNV showed reduced viral load in the brain, low levels of inflammatory molecules and less prominent neuropathology when compared to wild type mice, further suggesting the potential role of TLR 3 in viral entry into brain and neuropathology [73]. Conversely, a protective role of TLR3 with WNV infection has also been reported shedding doubt on the exact role of TLR3 in flavivirus pathogenesis [75]. In a separate study, activation of TLR3 was reported to block DENV type 2 replication via induction of IFN- $\beta$ in human hepatoma cell line [74].

It is consistently reported that TLR3 was activated by ZIKV possibly by sensing the replication intermediate of viral RNA [35, 38]. TLR3 was shown to be activated by ZIKV 
in human organoid cells and murine neurospheres, causing perturbation of 41 genes related to neurodevelopment and reducing the organoid volume as seen in clinical microcephaly [38]. The reduction in the organoid volume was correlated with the viral titers, indicating that activated TLR3 enhances ZIKV replication. Inhibition of TLR3/dsRNA by a competitive inhibitor (thiophenecarboxamidopropionate) resulted in a reversal of these phenotypic effects, while treatment with TLR3 agonist (poly I: C) mimicked ZIKV infection in terms of brain organoid volume [38]. It is paradoxical that a component of the innate immune response enhances viral replication and related pathogenesis. A proposed mechanism for ZIKV induced microcephaly suggested that ZIKV infection leads to TLR3 mediated hyper-activation of the innate immune response, which in turn cause transcriptional deregulation of the genes related to neurogenesis resulting in impaired neurogenesis [77].

\subsubsection{ZIKV and the autophagy pathway}

Autophagy mediates the lysosomal degradation of long-lived proteins, cellular organelles and intracellular pathogens [78]. The pathway is induced by nutrient deprivation or stress and promotes energy conservation and cell survival during starvation by recycling and salvage of cellular nutrients. Autophagy is a complex process involving more than 30 autophagy-related proteins (ATG) [79], crucial for cellular development and differentiation [80], innate and adaptive immunity [81, 82], and programmed cell death (type II) [83, 84]. Autophagosome formation involves initiation, nucleation, and expansion of the isolation membrane [85] and begins with the formation of the phagophore assembly site (PAS), the origin of which is still unclear in mammals [86, 87] (Figure 3). The autophagosome fuses with endocytic and lysosomal 
compartments to form auto-endosome or autolysosome for subsequent degradation [85]. Many signaling molecules such as mammalian target of rapamycin complex 1 (mTORC1), AMP-activated protein kinase (AMPK), death-associated protein kinase (DAPK), c-Jun $\mathrm{N}$ terminal kinase (JNK), Akt or protein kinase B (PKB), Casein kinase 2 (CK2), Insulin-like growth factor (IGF-1), DNA damage-regulated autophagy modulator (DRAM), p53, Forkhead box O (FOXO) and reactive oxygen species (ROS) are involved in autophagy regulation at various points [79].

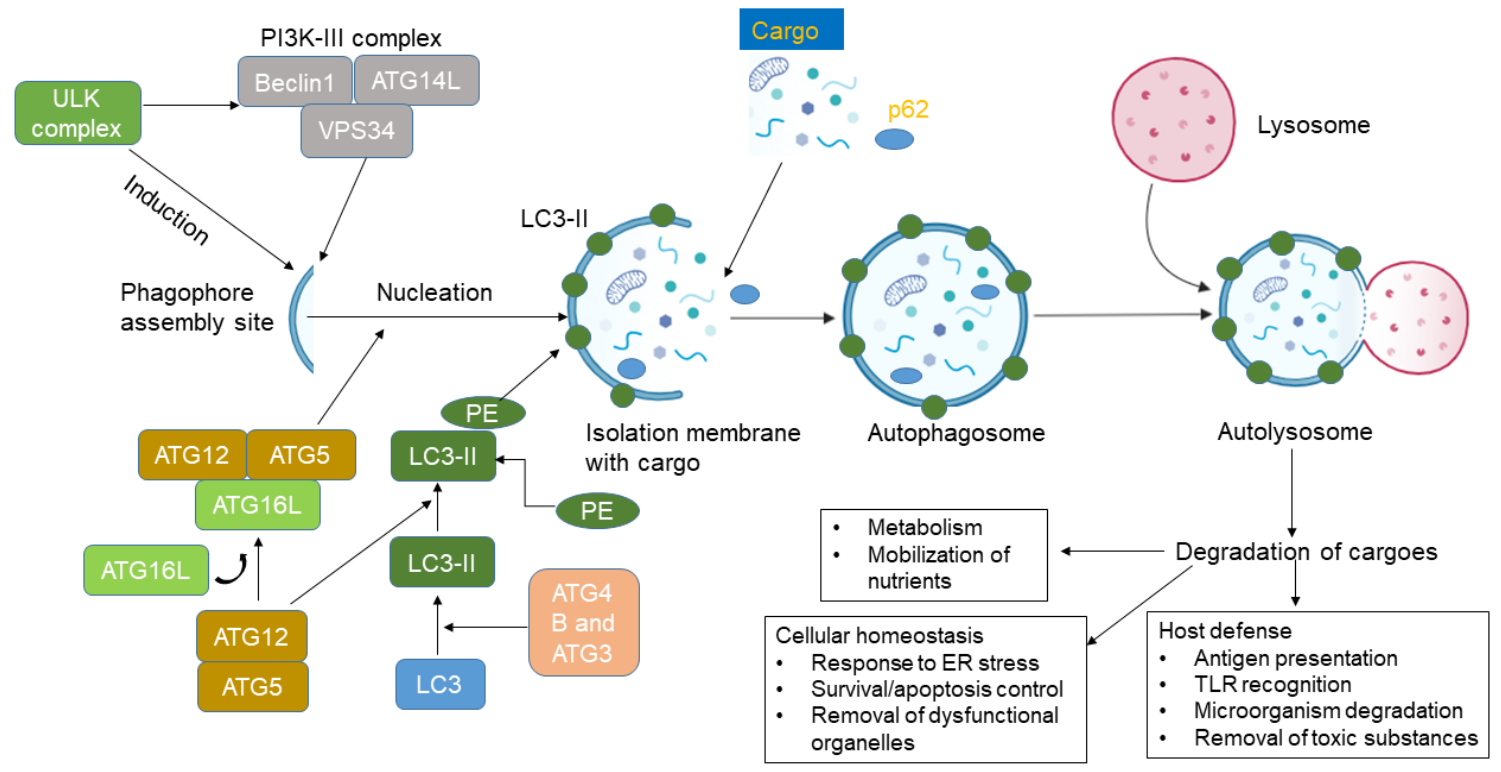

Figure 3. The major steps in the autophagy pathway.

The major steps in the autophagy pathway. The process of autophagy induction starts with the formation of UNC-51-like (ULK) complex comprising ULK-1, ATG13, ATG101, and FIP200. Beclin1 dependent enzymatic activity of a phosphatidylinositol 3-phosphate kinase 34 (VPS34) generates the phosphatidylinositol 3-phosphate, a substrate for autophagosome formation. The microtubule-associated protein light chain 3 (LC3) is cleaved and conjugated to phosphatidylethanolamine (PE) by sequencing action of AT4B, ATG3 and ATG12-ATG5 complex and is localized to the phagophore to facilitate autophagosome formation. P62, an adaptor protein also known as sequestosome 1(SQSTM1), targets specific cargoes towards autophagosome. Finally, the autophagosome fuses with the lysosome for the degradation of the cargoes. 
Microtubule-associated protein light chain-3 (LC3) and sequestosome-1 (SQSTM1, also called p62) are two important markers of autophagosome formation and its maturation $[88,89]$. During autophagosome formation, the cytosolic form of LC3 (LC3I) conjugates with phosphatidylethanolamine to form LC3-II. The lipidated form (LC3-II) is recruited to the autophagosome and gets degraded upon fusion with the lysosome in autolysosome, along with other damaged cellular components [88]. Induction of autophagy as determined by conversion of LC3-I to LC3-II and formation of LC3 punctate was recorded in fetal neuroprogenitor cells (fNSCs) infected with strains of ZIKV (MR766 and $\mathrm{IbH} 30656)$ in presence or absence of the lysosome inhibitor, bafilomycin A1 (Baf A1). p62/SQSTM1 is a ubiquitin-binding protein serving as an adaptor for the autophagy pathway by facilitating the delivery of cargo to autophagosomes [89]. A decrease in the p62/SQSTM1 levels with ZIKV infection was reported in fNSCs, indicating that autophagy maturation and lysosomal fusion were most likely not impaired.

On the other hand, inhibiting the autophagy pathway with pharmacological inhibitors 3-methyladenine (3-MA) or chloroquine in both fNSCs and HeLa cells or gene silencing targeting the autophagy-related gene (ATG)-3 in ZIKV-infected mouse embryo fibroblast (MEF) cells resulted in a staggering decrease in viral replication. Similar results were reported in siRNA-mediated inhibition of ATG-5 in MEF cells and siRNA-mediated inhibition of ATG-3 and ATG-13 in fNSCs [40], while induction of autophagy with rapamycin promoted ZIKV replication in both fNSCs and HeLa cells. These findings present sustainable evidence that ZIKV infection induces autophagy in fNSCs, which in turn leads to increased ZIKV replication and viral load [40]. Transient or stable expression of the NS4A and NS4B proteins in HeLa and fNPCs significantly induced the autophagy 
pathway, potentially through inhibition of the Akt-mTOR signaling pathway [40]. Studies have also shown that autophagy-related proteins play a significant role in placental defense against pathogenic microorganisms $[90,91]$.

Although the exact mechanisms by which ZIKV crosses the placental barrier is still not clear, it is speculated that ZIKV-packaged exosomes derived from trophoblastic cells may mediate placental transfer. This process is similar to secretory or unconventional autophagy, in which viral polypeptides that lack N-terminal peptides are unable to be secreted from the ER to the Golgi secretory pathway and are instead exported via secretory autophagy pathway [92]. Exosomes derived from ZIKV infected HEK293 cells show colocalization of the viral protein NS1 with the exosome markers CD63 and CD9, demonstrating that NS1 is present in the exosome. Exosomes derived from HEK293 cells transfected with NS1 plasmids further validated the presence of NS1 in exosomes. As with the vesicular stomatitis virus (VSV), placenta-specific microRNAs packaged in exosomes secreted from human syncytium may also induce the autophagy pathway and modulate ZIKV replication [93]. LC3-II protein expression was significantly upregulated at 6 and 12 hours-post-infection with ZIKV in human cytotrophoblast cells and further enhanced with Baf A1 [91]. Treatment with the pharmacological inhibitors of autophagy (3-MA, chloroquine, and Baf $\mathrm{A} 1)$ resulted in a significant decrease in ZIKV replication. The autophagy inducers, rapamycin and torin 1 caused a reciprocal increase in viral replication [91]. Mice deficient for the autophagy-related gene Atg16L1 infected with ZIKV showed improved fetal outcomes while treatment with hydroxychloroquine in pregnant dams caused reduced ZIKV vertical transmission and limited placental damage and fetal death 
[90]. Overall evidence support that the autophagy plays a crucial role in ZIKV replication and vertical transmission and can be targeted for therapeutic interventions against ZIKV.

\subsection{ZIKV animal research model and challenges}

So far several animal models have been proposed for ZIKV infection, including immunocompetent mice (C57BL/6), mice lacking the receptors for interferon, mice neutralized with the IFNAR1 antibodies, mice lacking IRF3/5/7, immunocompetent nonhuman primates and chicken embryos [94] and [95]. The most commonly employed mice models are the A129 strain which lacks the receptors for type 1 interferon and the AG129 strain which lacks the receptors for both type 1 and 2 IFN [96-98]. Comparable results can be achieved when using C57BL/6 mice treated with IFNAR1 monoclonal antibody before and during viral infection or using $\operatorname{Irf3} 3^{-/-} \operatorname{Irf} 5^{-/-} \operatorname{Irf}^{-/-}$triple knockout mice $[25,96]$. Despite many advances in using ZIKV-infected mouse model in establishing the cell tropism, viral replication kinetics, host immune response, teratogenicity, and clinical manifestation, limited progress toward vaccine development has been made [95, 99]. Structural and immunological dissimilarities between human and mice placenta, resistance of immunocompetent mice to ZIKV replication, skepticism about the relevance of data generated from immune compromised or genetically modified mice, limited availability of non-human primates and associated high cost are some of the limitations that are currently manifesting with the use of animal models [94].

\subsection{Vaccine and therapy development approach for ZIKV}

Multiple vaccine platforms and approaches are being employed for ZIKV including DNA vaccines, subunit vaccines, whole inactivated virus vaccines and vectored vaccines [100]. The nucleoside-modified mRNA encoding the pre-membrane and envelope (prM- 
E) glycoproteins encapsulated in lipid nanoparticle (mRNA-LNP) and ZIKV prM/E sequence DNA vaccine demonstrated robust protection against ZIKV by eliciting strong neutralizing antibody responses both in mice and non-human primates [99, 101]. Some DNA vaccines are currently entering phase I clinical trial or clinical evaluation [102, 103]. Some of the current approaches under evaluation are targeting viral proteins including, the envelope protein to block cellular entry and fusion, the NS5 protein to inhibit its RNAdependent RNA polymerase and methyltransferase activities and the NS3 protein to inhibit protease and helicase activity [104-106]. Repurposing therapy including the use of sofosbuvir, kitasamycin, lovastatin-azauridine, palonosetron, and 5-fluorouracil are some potential drugs under consideration for ZIKV treatment [107-109]. Another approach for developing ZIKV treatment and vaccines is to target host cellular molecules that are associated with increased viral replication and viral-induced inflammation. Many of the putative molecular targets identified include AXL, TLR3, small inducible cytokine B10 (IP10), interferon-stimulated genes, IL-6 (Interleukin-6) and Eukaryotic translation initiation factor (eIF)-2A protein kinase (eIF2AK2) [110]. Targeted immunotherapy designed to boost the natural defenses against the pathogens can also combat ZIKV infection and associated complications. Monoclonal antibodies against various epitopes of ZIKV structural proteins, isolated from B lymphocytes of infected human or animals have shown potent neutralizing activity against ZIKV [111]. Cytotoxic T lymphocytes (CD8 $\left.{ }^{+}\right)$ from infected mice also offer protection against ZIKV and prevent pathogenesis of ZIKV infection [112]. The finding that ZIKV inhibits interferon signaling by targeting human STAT2 challenges the therapeutic implication of interferons as the inhibitors of ZIKV replication $[68,113]$. 


\subsection{Overview of neuron and glial cells}

The main cell types in the brain are neurons and glial cells. A neuron consists of a cell body, an axon, and many dendrites. Neurons are the core components and the basic functional units of the brain. The microtubule associated protein 2 (MAP-2), neuronspecific enolase (NSE) and calbindin are some of the molecular markers of the neuron [114]. Glial cells, the supporting cells in the brain, are of three types, astrocytes, oligodendrocytes and microglia. Astrocytes are the major cell types with star-shaped morphology and expression of intermediated filament glial fibrillary acidic proteins (GFAP). Astrocytes are involved a multitude of functions including in supporting neurons, support endothelial cells forming $\mathrm{BBB}$, reinforcing synapses, mechanical repair, and scarring of the brain, etc. Oligodendrocytes form the myelin sheath of the neuronal axon in CNS analogous to Schwan cells in the peripheral nervous system. Microglia are the resident macrophages in the brain generally remain immunologically inactive called ramified state. Various neuroinflammatory diseases, proinflammatory cytokines, and physical injuries can trigger microglial activation. Activated microglia can act as antigenpresenting, cytotoxic and phagocytic cells [115]. The interactions between neurons and glial cells are depicted in Figure 4.

Mammalian CNS is developed from the neural tube containing neural stem cells (NSCs). Early NSCs are called neuroepithelial cells, which differentiate into radial glial cells (RGCs) with an elongated radial shape. The RGCs, which reside in the embryonic ventricular zone, are the principal stem cells for neuronal development. RGCs are differentiated into neurons via the intermediate step of neuronal precursor cells [116]. Thus formed neurons migrate towards their destinations such as a cortical plate to form cerebral 
cortex, followed by generating neural circuity with axons and dendrites. Mouse cortical neurogenesis generally takes place between embryonic day (E.D.) 11 to17. Gliogenesis, the process of generation of glial cells generally follows neurogenesis in the fetal brain through the differentiation of RGCs into radial glia progenitors (RGPs) [117]. Neurogenesis is almost complete before birth with negligible production of neurons from post-natal NSCs. However, glial genesis and generation of neuronal processes continue until adulthood, increasing the brain volume [118].

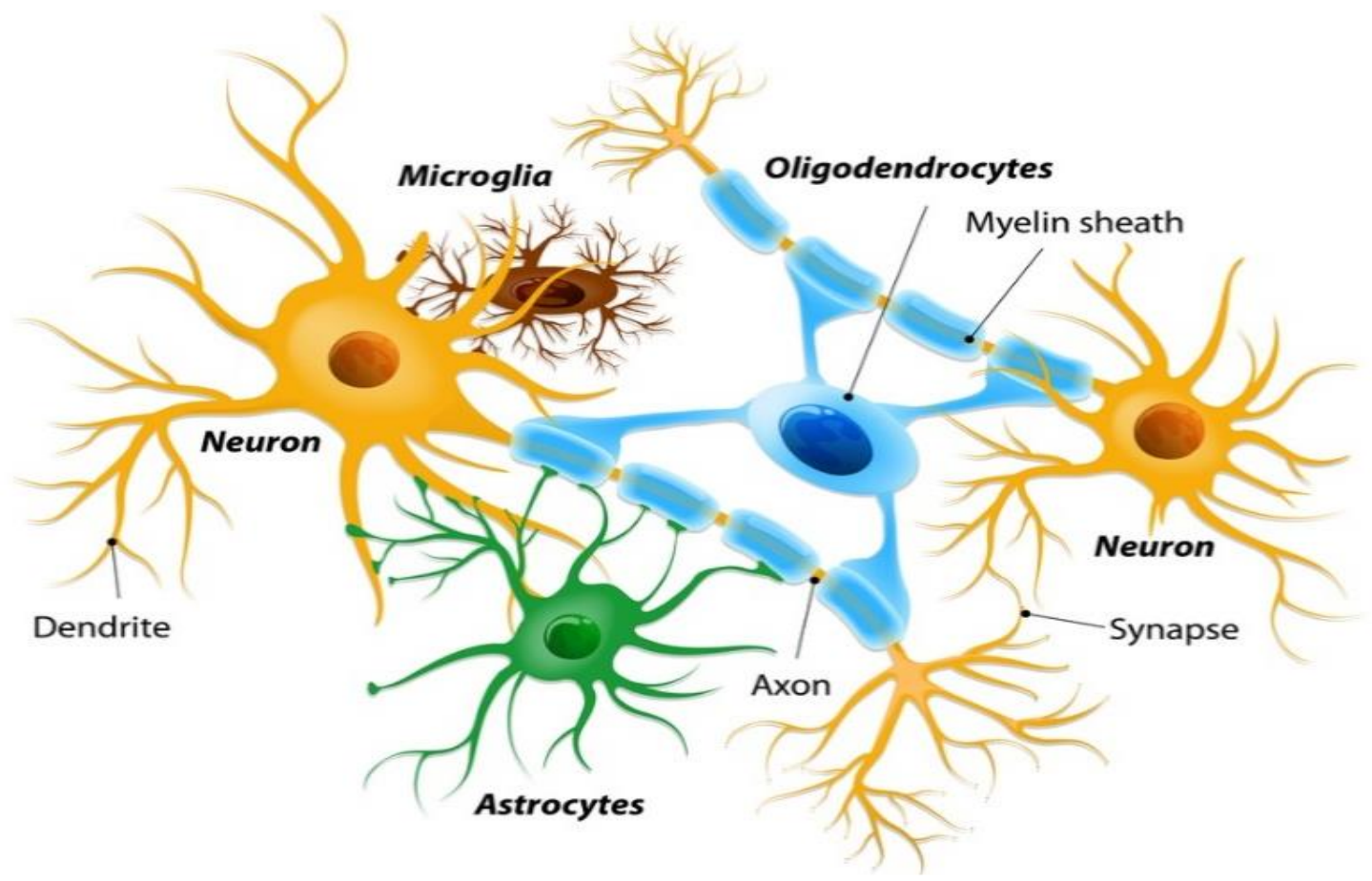

Figure 4. Interactions between cells of the central nervous system. Adapted from Parkinson's news today 17.

\subsection{Statement of the problem and objective of the study}

The first cases of ZIKV infection in the Americas occurred in Brazil in 2015, and since then an estimated 440,000 to 1,300,000 ZIKV infections are reported [119]. The virus has spread throughout South and Central Americas, including the Caribbean Islands, Puerto 
Rico and the southern part of the US [120]. Increased cases of fetal malformations such as spontaneous abortion, stillbirth, hydrocephaly, and microcephaly, and placental insufficiency to miscarriage, now collectively known as congenital Zika syndrome (CZS), were detected in Brazilian pregnant mothers infected with ZIKV during gestation [119]. Because of the size of the outbreak and severity of associated congenital defects, the World Health Organization (WHO) declared ZIKV to be a Public Health Emergency of International Concern (PHEIC) in February 2016. Though ZIKV is no more the PHEIC, many aspects of ZIKV infection and associated pathogenesis remain to be explored to develop effective vaccine and treatment strategies.

Mechanism of Zika viral entry into the target cell and transmission from mother to fetus is still not clear. Confirmation of the mechanism of viral entry will pave the way for a therapeutic target for ZIKV. Since many viruses including flaviviruses subvert autophagy to promote different stages of the viral life cycle [121], modulation of autophagy or Beclin1 protein could be an alternative approach for ZIKV therapy. Likewise, TLR3, a key modulator of the innate immune response against RNA viruses, could be another attractive target for developing a therapy against flaviviruses $[73,122]$. To our knowledge, this is the first report that compares the glial-pathogenesis along with the molecular mechanism among three spatiotemporally different ZIKV strains in the brain. The current study is designated to explore the molecular mechanism involved in ZIKV infection and at the same time compare the infection, pathology, and mechanism associated with spatiotemporally different ZIKV strains. To these ends, we proposed the following specific aims:

Specific Aim 1: Determine the infectivity and the mechanism of ZIKV entry into the brain cells. 
Specific Aim 2: Examine the molecular mechanism(s) of ZIKV infection and associated inflammatory responses in vitro focusing on ER stress, TLR and autophagy pathways.

Specific Aim 3: Explore the molecular mechanism of ZIKV infection and associated obstruction in brain development using an infectious mouse model.

\section{References}

[1] Kuno G, Chang GJ, Tsuchiya KR, Karabatsos N, Cropp CB. Phylogeny of the genus Flavivirus. J Virol. 1998;72(1):73-83.

[2] Dick GWA, Kitchen SF, Haddow AJ. Zika Virus (I). Isolations and serological specificity. Transactions of The Royal Society of Tropical Medicine and Hygiene. 1952;46(5):509-20.

[3] Smithburn KC. Neutralizing antibodies against certain recently isolated viruses in the sera of human beings residing in East Africa. Journal of immunology (Baltimore, Md : 1950). 1952;69(2):223-34.

[4] de Araújo TVB, Rodrigues LC, de Alencar Ximenes RA, de Barros MirandaFilho D, Montarroyos UR, de Melo APL, et al. Association between Zika virus infection and microcephaly in Brazil, January to May, 2016: preliminary report of a case-control study. The Lancet Infectious Diseases. 2016;16(12):1356-63.

[5] Parra B, Lizarazo J, Jimenez-Arango JA, Zea-Vera AF, Gonzalez-Manrique G, Vargas J, et al. Guillain-Barre Syndrome Associated with Zika Virus Infection in Colombia. N Engl J Med. 2016;375(16):1513-23.

[6] Cao-Lormeau V-M, Blake A, Mons S, Lastère S, Roche C, Vanhomwegen J, et al. Guillain-Barr\&\#xe9; Syndrome outbreak associated with Zika virus infection in French Polynesia: a case-control study. The Lancet. 387(10027):1531-9.

[7] Mendez N, Oviedo-Pastrana M, Mattar S, Caicedo-Castro I, Arrieta G. Zika virus disease, microcephaly and Guillain-Barre syndrome in Colombia: epidemiological situation during 21 months of the Zika virus outbreak, 2015-2017. Archives of public health $=$ Archives belges de sante publique. 2017;75:65.

[8] Cauchemez S, Besnard M, Bompard P, Dub T, Guillemette-Artur P, EyrolleGuignot D, et al. Association between Zika virus and microcephaly in French Polynesia, 2013-15: a retrospective study. The Lancet. 2016;387(10033):212532. 
[9] Althaus CL, Low N. How Relevant Is Sexual Transmission of Zika Virus? PLOS Medicine. 2016;13(10):e1002157.

[10] Brian DF, Kevin CK, Joy LCF, Bradley JB, Amelia Travassos da R, Andrew DH, et al. Probable Non-Vector-borne Transmission of Zika Virus, Colorado, USA. Emerging Infectious Disease journal. 2011;17(5):880.

[11] Dupont-Rouzeyrol M, Biron A, O'Connor O, Huguon E, Descloux E. Infectious Zika viral particles in breastmilk. Lancet. 2016;387(10023):1051.

[12] Hirsch AJ, Roberts VHJ, Grigsby PL, Haese N, Schabel MC, Wang X, et al. Zika virus infection in pregnant rhesus macaques causes placental dysfunction and immunopathology. Nature Communications. 2018;9(1):263.

[13] Miner JJ, Diamond MS. Zika virus pathogenesis and tissue tropism. Cell host \& microbe. 2017;21(2):134-42.

[14] Baronti C, Piorkowski G, Charrel RN, Boubis L, Leparc-Goffart I, de Lamballerie X. Complete Coding Sequence of Zika Virus from a French Polynesia Outbreak in 2013. Genome Announcements. 2014;2(3):e00500-14.

[15] van Hemert F, Berkhout B. Nucleotide composition of the Zika virus RNA genome and its codon usage. Virology Journal. 2016;13(1):95.

[16] Cunha MS, Esposito DLA, Rocco IM, Maeda AY, Vasami FGS, Nogueira JS, et al. First Complete Genome Sequence of Zika Virus (Flaviviridae, Flavivirus) from an Autochthonous Transmission in Brazil. Genome Announcements. 2016;4(2):e00032-16.

[17] Suthar MS, Diamond MS, Gale M, Jr. West Nile virus infection and immunity. Nature reviews Microbiology. 2013;11(2):115-28.

[18] Pierson TC, Kielian M. Flaviviruses: braking the entering. Current opinion in virology. 2013;3(1):3-12.

[19] Chung KM, Diamond MS. Defining the levels of secreted non-structural protein NS1 after West Nile virus infection in cell culture and mice. J Med Virol. 2008;80(3):547-56.

[20] Mukhopadhyay S, Kuhn RJ, Rossmann MG. A structural perspective of the flavivirus life cycle. Nature reviews Microbiology. 2005;3(1):13-22. 
[21] Dai L, Song J, Lu X, Deng Y-Q, Musyoki AM, Cheng H, et al. Structures of the Zika Virus Envelope Protein and Its Complex with a Flavivirus Broadly Protective Antibody. Cell host \& microbe. 2016;19:696-704.

[22] Faye O, Freire CC, Iamarino A, Faye O, de Oliveira JV, Diallo M, et al. Molecular evolution of Zika virus during its emergence in the 20(th) century. PLoS Negl Trop Dis. 2014;8(1):e2636.

[23] Haddow AD, Schuh AJ, Yasuda CY, Kasper MR, Heang V, Huy R, et al. Genetic Characterization of Zika Virus Strains: Geographic Expansion of the Asian Lineage. PLOS Neglected Tropical Diseases. 2012;6(2):e1477.

[24] Tripathi S, Balasubramaniam VRMT, Brown JA, Mena I, Grant A, Bardina SV, et al. A novel Zika virus mouse model reveals strain specific differences in virus pathogenesis and host inflammatory immune responses. PLOS Pathogens. 2017;13(3):e1006258.

[25] Lazear Helen M, Govero J, Smith Amber M, Platt Derek J, Fernandez E, Miner Jonathan J, et al. A Mouse Model of Zika Virus Pathogenesis. Cell Host \& Microbe. 19(5):720-30.

[26] Simonin Y, Loustalot F, Desmetz C, Foulongne V, Constant O, Fournier-Wirth C, et al. Zika Virus Strains Potentially Display Different Infectious Profiles in Human Neural Cells. EBioMedicine. 2016;12:161-9.

[27] Smit JM, Moesker B, Rodenhuis-Zybert I, Wilschut J. Flavivirus Cell Entry and Membrane Fusion. Viruses. 2011;3(2):160-71.

[28] Meertens L, Labeau A, Dejarnac O, Cipriani S, Sinigaglia L, Bonnet-Madin L, et al. Axl Mediates ZIKA Virus Entry in Human Glial Cells and Modulates Innate Immune Responses. Cell Reports. 2017;18(2):324-33.

[29] Chen J, Yang Y-f, Yang Y, Zou P, Chen J, He Y, et al. AXL promotes Zika virus infection in astrocytes by antagonizing type I interferon signalling. Nature Microbiology. 2018;3(3):302-9.

[30] O'Bryan JP, Frye RA, Cogswell PC, Neubauer A, Kitch B, Prokop C, et al. axl, a transforming gene isolated from primary human myeloid leukemia cells, encodes a novel receptor tyrosine kinase. Molecular and Cellular Biology. 1991;11(10):5016-31.

[31] Paolino M, Penninger JM. The Role of TAM Family Receptors in Immune Cell Function: Implications for Cancer Therapy. Cancers. 2016;8(10):97. 
[32] Rankin EB, Giaccia AJ. The Receptor Tyrosine Kinase AXL in Cancer Progression. Cancers. 2016;8(11):103.

[33] Camenisch TD, Koller BH, Earp HS, Matsushima GK. A novel receptor tyrosine kinase, Mer, inhibits TNF-alpha production and lipopolysaccharide-induced endotoxic shock. Journal of immunology (Baltimore, Md : 1950). 1999;162(6):3498-503.

[34] Jung CY, Kim SY, Lee C. Carvacrol Targets AXL to Inhibit Cell Proliferation and Migration in Non-small Cell Lung Cancer Cells. Anticancer research. 2018;38(1):279-86.

[35] Hamel R, Dejarnac O, Wichit S, Ekchariyawat P, Neyret A, Luplertlop N, et al. Biology of Zika Virus Infection in Human Skin Cells. journal of virology. 2015;89(17):8880-96.

[36] Barber GN. Host defense, viruses and apoptosis. Cell death and differentiation. 2001;8(2):113-26.

[37] Thompson MR, Kaminski JJ, Kurt-Jones EA, Fitzgerald KA. Pattern Recognition Receptors and the Innate Immune Response to Viral Infection. Viruses. 2011;3(6):920-40.

[38] Dang J, Tiwari SK, Lichinchi G, Qin Y, Patil VS, Eroshkin AM, et al. Zika Virus Depletes Neural Progenitors in Human Cerebral Organoids through Activation of the Innate Immune Receptor TLR3. Cell stem cell. 2016;19(2):258-65.

[39] Zhang F, Hammack C, Ogden SC, Cheng Y, Lee EM, Wen Z, et al. Molecular signatures associated with ZIKV exposure in human cortical neural progenitors. Nucleic Acids Res. 2016;44(18):8610-20.

[40] Liang Q, Luo Z, Zeng J, Chen W, Foo S-S, Lee S-A, et al. Zika Virus NS4A and NS4B Proteins Deregulate Akt-mTOR Signaling in Human Fetal Neural Stem Cells to Inhibit Neurogenesis and Induce Autophagy. Cell Stem Cell. 19(5):66371.

[41] Gillespie LK, Hoenen A, Morgan G, Mackenzie JM. The endoplasmic reticulum provides the membrane platform for biogenesis of the flavivirus replication complex. J Virol. 2010;84(20):10438-47. 
[42] Heaton NS, Perera R, Berger KL, Khadka S, Lacount DJ, Kuhn RJ, et al. Dengue virus nonstructural protein 3 redistributes fatty acid synthase to sites of viral replication and increases cellular fatty acid synthesis. Proc Natl Acad Sci U S A. 2010;107(40):17345-50.

[43] Miller S, Kastner S, Krijnse-Locker J, Buhler S, Bartenschlager R. The nonstructural protein $4 \mathrm{~A}$ of dengue virus is an integral membrane protein inducing membrane alterations in a $2 \mathrm{~K}$-regulated manner. J Biol Chem. 2007;282(12):8873-82.

[44] Kaufusi PH, Kelley JF, Yanagihara R, Nerurkar VR. Induction of endoplasmic reticulum-derived replication-competent membrane structures by West Nile virus non-structural protein 4B. PLoS One. 2014;9(1):e84040.

[45] Blázquez AB, Escribano-Romero E, Merino-Ramos T, Saiz JC, Martín-Acebes MA. Stress responses in flavivirus-infected cells: activation of unfolded protein response and autophagy. Front Microbiol. 2014;5.

[46] Hetz C. The unfolded protein response: controlling cell fate decisions under ER stress and beyond. Nature Reviews Molecular Cell Biology. 2012;13:89.

[47] Liu CY, Schroder M, Kaufman RJ. Ligand-independent dimerization activates the stress response kinases IRE1 and PERK in the lumen of the endoplasmic reticulum. J Biol Chem. 2000;275(32):24881-5.

[48] Gladwyn-Ng I, Cordón-Barris L, Alfano C, Creppe C, Couderc T, Morelli G, et al. Stress-induced unfolded protein response contributes to Zika virus-associated microcephaly. Nature Neuroscience. 2018;21(1):63-71.

[49] Anderson P, Kedersha N. Stressful initiations. Journal of Cell Science. 2002;115(16):3227.

[50] Emara MM, Brinton MA. Interaction of TIA-1/TIAR with West Nile and dengue virus products in infected cells interferes with stress granule formation and processing body assembly. Proceedings of the National Academy of Sciences. 2007;104(21):9041-6.

[51] Khaperskyy DA, Hatchette TF, McCormick C. Influenza A virus inhibits cytoplasmic stress granule formation. The FASEB Journal. 2011;26(4):1629-39.

[52] Hou S, Kumar A, Xu Z, Airo AM, Stryapunina I, Wong CP, et al. Zika virus hijacks stress granule proteins and modulates the host stress response. J Virol. 2017. 
[53] Amorim R, Temzi A, Griffin BD, Mouland AJ. Zika virus inhibits eIF2alphadependent stress granule assembly. PLoS Negl Trop Dis. 2017;11(7):e0005775.

[54] Tang H, Hammack C, Ogden SC, Wen Z, Qian X, Li Y, et al. Zika Virus Infects Human Cortical Neural Precursors and Attenuates Their Growth. Cell stem cell. 2016;18(5):587-90.

[55] Stefanik M, Formanova P, Bily T, Vancova M, Eyer L, Palus M, et al. Characterisation of Zika virus infection in primary human astrocytes. BMC Neuroscience. 2018;19:5.

[56] Papa MP, Meuren LM, Coelho SVA, Lucas CGdO, Mustafá YM, Lemos Matassoli F, et al. Zika Virus Infects, Activates, and Crosses Brain Microvascular Endothelial Cells, without Barrier Disruption. Frontiers in Microbiology. 2017;8:2557.

[57] Roach T, Alcendor DJ. Zika virus infection of cellular components of the bloodretinal barriers: implications for viral associated congenital ocular disease. Journal of Neuroinflammation. 2017;14:43.

[58] Cumberworth SL, Barrie JA, Cunningham ME, de Figueiredo DPG, Schultz V, Wilder-Smith AJ, et al. Zika virus tropism and interactions in myelinating neural cell cultures: CNS cells and myelin are preferentially affected. Acta Neuropathologica Communications. 2017;5(1):50.

[59] Furtado JM, Espósito DL, Klein TM, Teixeira-Pinto T, da Fonseca BA. Uveitis Associated with Zika Virus Infection. New England Journal of Medicine. 2016;375(4):394-6.

[60] Winkler CW, Myers LM, Woods TA, Messer RJ, Carmody AB, McNally KL, et al. Adaptive Immune Responses to Zika Virus Are Important for Controlling Virus Infection and Preventing Infection in Brain and Testes. Journal of immunology (Baltimore, Md : 1950). 2017;198(9):3526-35.

[61] Stetson DB, Medzhitov R. Type I interferons in host defense. Immunity. 2006;25(3):373-81.

[62] Xie X, Shan C, Shi P-Y. Restriction of Zika Virus by Host Innate Immunity. Cell Host \& Microbe. 2016;19(5):566-7.

[63] Rossi SL, Tesh RB, Azar SR, Muruato AE, Hanley KA, Auguste AJ, et al. Characterization of a Novel Murine Model to Study Zika Virus. The American journal of tropical medicine and hygiene. 2016;94(6):1362-9. 
[64] Robbiani DF, Bozzacco L, Keeffe JR, Khouri R, Olsen PC, Gazumyan A, et al. Recurrent Potent Human Neutralizing Antibodies to Zika Virus in Brazil and Mexico. Cell. 2017;169(4):597-609.e11.

[65] Larocca RA, Abbink P, Peron JPS, de A. Zanotto PM, Iampietro MJ, BadamchiZadeh A, et al. Vaccine protection against Zika virus from Brazil. Nature. 2016;536:474.

[66] Pardy RD, Rajah MM, Condotta SA, Taylor NG, Sagan SM, Richer MJ. Analysis of the T Cell Response to Zika Virus and Identification of a Novel CD8+ T Cell Epitope in Immunocompetent Mice. PLOS Pathogens. 2017;13(2):e1006184.

[67] Winkler CW, Myers LM, Woods TA, Messer RJ, Carmody AB, McNally KL, et al. Adaptive Immune Responses To Zika Virus Are Important For Controlling Virus Infection And Preventing Infection In Brain And Testes. Journal of immunology 2017;198(9):3526-35.

[68] Grant A, Ponia SS, Tripathi S, Balasubramaniam V, Miorin L, Sourisseau M, et al. Zika virus targets human STAT2 to inhibit type I interferon signaling. Cell Host Microbe. 2016;19(6):882-90.

[69] Onorati M, Li Z, Liu F, Sousa AMM, Nakagawa N, Li M, et al. Zika Virus Disrupts Phospho-TBK1 Localization and Mitosis in Human Neuroepithelial Stem Cells and Radial Glia. Cell Rep. 2016;16(10):2576-92.

[70] Wu Y, Liu Q, Zhou J, Xie W, Chen C, Wang Z, et al. Zika virus evades interferon-mediated antiviral response through the co-operation of multiple nonstructural proteins in vitro. Cell Discov. 2017;3:17006-.

[71] Garcia M, Wehbe M, Lévêque N, Bodet C. Skin innate immune response to flaviviral infection. European Cytokine Network. 2017;28(2):41-51.

[72] Koyama S, Ishii KJ, Coban C, Akira S. Innate immune response to viral infection. Cytokine. 2008;43(3):336-41.

[73] Wang T, Town T, Alexopoulou L, Anderson JF, Fikrig E, Flavell RA. Toll-like receptor 3 mediates West Nile virus entry into the brain causing lethal encephalitis. Nature medicine. 2004;10(12):1366-73.

[74] Liang Z, Wu S, Li Y, He L, Wu M, Jiang L, et al. Activation of Toll-like receptor 3 impairs the dengue virus serotype 2 replication through induction of IFN-beta in cultured hepatoma cells. PLoS One. 2011;6(8):e23346. 
[75] Daffis S, Samuel MA, Suthar MS, Jr. MG, Diamond MS. Toll-Like Receptor 3 Has a Protective Role against West Nile Virus Infection. Journal of Virology. 2008.

[76] Goffic RL, Balloy V, Lagranderie M, Alexopoulou L, Escriou N, Flavell R, et al. Detrimental Contribution of the Toll-Like Receptor (TLR)3 to Influenza A VirusInduced Acute Pneumonia. PLOS Pathogens. 2006;2(6):e53.

[77] Faizan MI, Abdullah M, Ali S, Naqvi IH, Ahmed A, Parveen S. Zika VirusInduced Microcephaly and Its Possible Molecular Mechanism. Intervirology. 2016;59(3):152-8.

[78] Deter RL, De Duve C. Influence of glucagon, an inducer of cellular autophagy, on some physical properties of rat liver lysosomes. J Cell Biol. 1967;33(2):437-49.

[79] Ojha CR, Lapierre J, Rodriguez M, Dever SM, Zadeh MA, DeMarino C, et al. Interplay between Autophagy, Exosomes and HIV-1 Associated Neurological Disorders: New Insights for Diagnosis and Therapeutic Applications. Viruses. 2017;9(7).

[80] Tsukamoto S, Kuma A, Murakami M, Kishi C, Yamamoto A, Mizushima N. Autophagy is essential for preimplantation development of mouse embryos. Science. 2008;321(5885):117-20.

[81] Xu Y, Jagannath C, Liu XD, Sharafkhaneh A, Kolodziejska KE, Eissa NT. Tolllike receptor 4 is a sensor for autophagy associated with innate immunity. Immunity. 2007;27(1):135-44.

[82] Shibutani ST, Saitoh T, Nowag H, Munz C, Yoshimori T. Autophagy and autophagy-related proteins in the immune system. Nat Immunol. 2015;16(10):1014-24.

[83] Yu L, Alva A, Su H, Dutt P, Freundt E, Welsh S, et al. Regulation of an ATG7beclin 1 program of autophagic cell death by caspase-8. Science. 2004;304(5676):1500-2.

[84] Tsujimoto Y, Shimizu S. Another way to die: autophagic programmed cell death. Cell death and differentiation. 2005;12 Suppl 2:1528-34.

[85] Kaur J, Debnath J. Autophagy at the crossroads of catabolism and anabolism. Nature reviews Molecular cell biology. 2015;16(8):461-72.

[86] Glick D, Barth S, Macleod KF. Autophagy: cellular and molecular mechanisms. The Journal of pathology. 2010;221(1):3-12. 
[87] Lamb CA, Yoshimori T, Tooze SA. The autophagosome: origins unknown, biogenesis complex. Nature reviews Molecular cell biology. 2013;14(12):759-74.

[88] Tanida I, Ueno T, Kominami E. LC3 and Autophagy. Methods in molecular biology (Clifton, NJ). 2008;445:77-88.

[89] Rusten TE, Stenmark H. p62, an autophagy hero or culprit? Nature Cell Biology. 2010;12:207.

[90] Cao B, Macones C, Mysorekar IU. ATG16L1 governs placental infection risk and preterm birth in mice and women. JCI Insight. 2016;1(21).

[91] Cao B, Parnell LA, Diamond MS, Mysorekar IU. Inhibition of autophagy limits vertical transmission of Zika virus in pregnant mice. The Journal of Experimental Medicine. 2017;214(8):2303-13.

[92] Zhang Z-W, Li Z-L, Yuan S. The Role of Secretory Autophagy in Zika Virus Transfer through the Placental Barrier. Frontiers in Cellular and Infection Microbiology. 2016;6:206.

[93] Delorme-Axford E, Donker RB, Mouillet J-F, Chu T, Bayer A, Ouyang Y, et al. Human placental trophoblasts confer viral resistance to recipient cells. Proceedings of the National Academy of Sciences. 2013;110(29):12048-53.

[94] Morrison TE, Diamond MS. Animal Models of Zika Virus Infection, Pathogenesis, and Immunity. J Virol. 2017;91(8).

[95] Pawitwar SS, Dhar S, Tiwari S, Ojha CR, Lapierre J, Martins K, et al. Overview on the Current Status of Zika Virus Pathogenesis and Animal Related Research. Journal of neuroimmune pharmacology : the official journal of the Society on NeuroImmune Pharmacology. 2017;12(3):371-88.

[96] Smith DR, Hollidge B, Daye S, Zeng X, Blancett C, Kuszpit K, et al. Neuropathogenesis of Zika Virus in a Highly Susceptible Immunocompetent Mouse Model after Antibody Blockade of Type I Interferon. PLoS Negl Trop Dis. 2017;11(1).

[97] Aliota MT, Caine EA, Walker EC, Larkin KE, Camacho E, Osorio JE. Characterization of Lethal Zika Virus Infection in AG129 Mice. PLOS Neglected Tropical Diseases. 2016;10(4):e0004682.

[98] Lazear HM, Govero J, Smith AM, Platt DJ, Fernandez E, Miner JJ, et al. A Mouse Model of Zika Virus Pathogenesis. Cell host \& microbe. 2016;19(5):72030. 
[99] Larocca RA, Abbink P, Peron JP, Zanotto PM, Iampietro MJ, Badamchi-Zadeh A, et al. Vaccine protection against Zika virus from Brazil. Nature. 2016;536(7617):474-8.

[100] Dawes BE, Smalley CA, Tiner BL, Beasley DW, Milligan GN, Reece LM, et al. Research and development of Zika virus vaccines. npj Vaccines. 2016;1:16007.

[101] Pardi N, Hogan MJ, Pelc RS, Muramatsu H, Andersen H, DeMaso CR, et al. Zika virus protection by a single low-dose nucleoside-modified mRNA vaccination. Nature. 2017;543(7644):248.

[102] Dyer O. Trials of Zika vaccine are set to begin in North America. Bmj. 2016;353:i3588.

[103] Barrett ADT. Zika vaccine candidates progress through nonclinical development and enter clinical trials. npj Vaccines. 2016;1:16023.

[104] Panayiotou C, Lindqvist R, Kurhade C, Vonderstein K, Pasto J, Edlund K, et al. Viperin restricts Zika virus and tick-borne encephalitis virus replication by targeting NS3 for proteasomal degradation. Journal of Virology. 2018.

[105] Yang M, Lai H, Sun H, Chen Q. Virus-like particles that display Zika virus envelope protein domain III induce potent neutralizing immune responses in mice. Scientific Reports. 2017;7(1):7679.

[106] Ramharack P, Soliman MES. Zika virus NS5 protein potential inhibitors: an enhanced in silico approach in drug discovery. Journal of Biomolecular Structure and dynamics. 2017.

[107] Eyer L, Nencka R, Huvarova I, Palus M, Joao Alves M, Gould EA, et al. Nucleoside Inhibitors of Zika Virus. J Infect Dis. 2016;214(5):707-11.

[108] Bullard-Feibelman KM, Govero J, Zhu Z, Salazar V, Veselinovic M, Diamond MS, et al. The FDA-approved drug sofosbuvir inhibits Zika virus infection. Antiviral Res. 2017;137:134-40.

[109] Abrams RPM, Solis J, Nath A. Therapeutic Approaches for Zika Virus Infection of the Nervous System. Neurotherapeutics : the journal of the American Society for Experimental NeuroTherapeutics. 2017;14(4):1027-48.

[110] Ramaswamy Narayanan*. Zika Virus Therapeutics: Drug Targets and Repurposing Medicine from the Human Genome. MOJ Proteomics \& Bioinformatics. 2018;3(3):1-7. 
[111] Sautto G, Mancini N, Gorini G, Clementi M, Burioni R. Possible future monoclonal antibody (mAb)-based therapy against arbovirus infections. Biomed Res Int. 2013;2013:838491.

[112] Heslop HE, Leen AM. T-cell therapy for viral infections. Hematology American Society of Hematology Education Program. 2013;2013:342-7.

[113] Contreras D, Arumugaswami V. Zika Virus Infectious Cell Culture System and the In Vitro Prophylactic Effect of Interferons. J Vis Exp. 2016;(114).

[114] Lodish H, Berk A, Zipursky SL, Matsudaira P, Baltimore D, Darnell J. Overview of Neuron Structure and Function. Molecular cell biology 4th edition. Newyork: W. H. Freeman; 2000.

[115] von Bernhardi R, Eugenín-von Bernhardi J, Flores B, Eugenín León J. Glial Cells and Integrity of the Nervous System. In: von Bernhardi R, editor. Glial Cells in Health and Disease of the CNS. Cham: Springer International Publishing; 2016. p. $1-24$.

[116] Noctor SC, Martínez-Cerdeño V, Ivic L, Kriegstein AR. Cortical neurons arise in symmetric and asymmetric division zones and migrate through specific phases. Nature Neuroscience. 2004;7:136.

[117] Freeman Marc R, Rowitch David H. Evolving Concepts of Gliogenesis: A Look Way Back and Ahead to the Next 25 Years. Neuron. 2013;80(3):613-23.

[118] Gilmore EC, Walsh CA. Genetic Causes of Microcephaly and Lessons for Neuronal Development. Wiley interdisciplinary reviews Developmental biology. 2013;2(4):461-78.

[119] Thomas Jaenisch KDR, Carlos Brito, Oliver Brady, Patrícia Brasil. Risk of microcephaly after Zika virus infection in Brazil, 2015 to 2016. Bulletin of World Health Organization. 2017;95:191-8.

[120] Sampathkumar P, Sanchez JL. Zika Virus in the Americas: A Review for Clinicians. Mayo Clinic proceedings. 2016;91(4):514-21.

[121] Carneiro LAM, Travassos LH. Autophagy and viral diseases transmitted by Aedes aegypti and Aedes albopictus. 2015.

[122] Xagorari A, Chlichlia K. Toll-Like Receptors and Viruses: Induction of Innate Antiviral Immune Responses. Open Microbiol J. 2008;2:49-59. 


\section{CHAPTER 2: ZIKV INFECTION IN BRAIN CELLS INDUCES INFLAMMATORY MOLECULES SECRETION AND CELL DEATH}

\subsection{Introduction}

A causal association of ZIKV with severe neurodevelopmental and neurodegenerative disorders prompted the scientific community to investigate the mechanism of ZIKV-induced neuropathogenesis [1, 2]. However, most of the studies are focusing on the direct impact of ZIKV on the neuroprogenitor cells, and the potential role of differentiated neurons and glial cells in ZIKV-induced neuropathologies is overlooked [3, 4]. Glial cells including astrocytes and microglia are the most important targets for ZIKV pathology in the brain [5, 6]. Astrocytes, the most abundant glial cells are located in the vicinity of capillaries and are a key component of the blood-brain barrier (BBB). Therefore, astrocytes may be the first target encountered by ZIKV immediately after entering the central nervous system. Microglia, the resident macrophages in the central nervous system (CNS), have an important role in neuronal development and homeostasis [7]. Glial cell infection by ZIKV may cause neuroinflammation by releasing proinflammatory molecules which lead to BBB leakage and further exacerbation of the pathogenesis [8]. Despite the crucial role of astrocytes and microglia in ZIKV infection, little is known about the interaction of ZIKV with the glial in terms of viral kinetics, inflammatory profiles and cellular toxicity $[5,6]$.

Genetic and phylogenetic studies reveal that ZIKV has evolved into different lineages including the African lineage comprising of the West African (Nigerian) and the East African (MR766 prototype) clusters, and the Asian lineage including Polynesian and Brazilian clusters $[9,10]$. Genetic changes among the ZIKV lineage strains may attribute 
to the global spread of a new phenotype and the emergence of more neuro-virulent strains [10]. Comparative studies of different ZIKV strains have shown inconsistent reports in terms of viral lethality, cellular infectivity, antiviral responses and genetic changes $[3,10$ $13]$.

The goal of the studies proposed in Aim 1 was to compare the infectivity levels of spatiotemporally different strains of ZIKV in different brain cells and associated neuropathology. We also investigated the viral replication kinetics and inflammatory molecules secretion profiles. Here we report that ZIKV infects major cell types in the CNS including neurons, astrocytes, and microglia. Our findings show that viral strains vary in their capacity to infect these cells, to cause cell death and to induce a release of inflammatory molecules.

\subsection{Materials and methods}

\subsubsection{Zika virus propagation and isolation}

Zika virus strains MR766 (Cat. VR84, ATCC, Manassas, VA, USA), R103451 (Cat. VR-1848, ATCC, Manassas, VA, USA) and PRVABC59 (Cat. VR-1843, ATCC, Manassas, VA, USA) were each propagated in Vero cells (Cat. CRL-1586, ATCC, Manassas, VA, USA) and/or mosquito cell line C6/36 (Cat. CRL-1660, ATCC, Manassas, VA, USA). Briefly, Vero cells or C6/36 cells were grown in T75 flasks with recommended growth media (EMEM supplemented with 10\% FBS and 1\% antibiotic/antimycotic solution) to $>80 \%$ confluency and infected with $4 \mathrm{ml}$ of ZIKV (diluted in EMEM) at a multiplicity of infection (MOI) of 0.01 . Culture flasks were incubated at $37^{\circ} \mathrm{C}$ and $5 \% \mathrm{CO}_{2}$ for 2 hours with gentle rocking every 15 minutes, followed by addition of $4 \mathrm{ml}$ of media and continuous incubation for 5 days. The supernatant was harvested after 5 days, 
centrifuged and filtered using 0.45-micron filter and aliquoted in $2.0 \mathrm{ml}$ cryotubes [14]. Virus titer was determined by standard plaque assay and further amplified by propagating in $\mathrm{C} 6 / 36$ mosquito cells cultured in EMEM supplemented with $10 \% \mathrm{FBS}$ at $28^{\circ} \mathrm{C}$ and $5 \%$ $\mathrm{CO}_{2}$.

\subsubsection{Viral quantification by plaque assay}

Vero cells were infected with a ten-fold dilution of ZIKV stock or the supernatants from infected/treated cells for 1 hour to allow adsorption and then washed with phosphate buffer saline (PBS). Cells were overlaid with culture media (EMEM supplemented with $2 \%$ FBS) containing an equal volume of $3.2 \%$ carboxymethylcellulose (CMC) and incubated for 5 days at $37^{\circ} \mathrm{C}$. Cells were fixed and stained with $1 \%$ crystal violet solution prepared in $20 \%$ formaldehyde, $30 \%$ ethanol and 50\% PBS for 1 hour. Stained cells were washed with water to remove excess crystal violet, left to dry overnight, and the lysis plaques were quantified by stereomicroscope (Figure 5A and B).

\subsubsection{Cell cultures and ZIKV infection}

Human primary astrocytes (Cat. 1800, ScienCell, Carlsbad, CA, USA) were grown and maintained in astrocyte medium supplemented with $2 \%$ FBS, $1 \%$ astrocyte growth supplement (AGS) and 1\% antibiotic/antimycotic (penicillin/streptomycin (P/S)) solution (ScienCell, Carlsbad, CA, USA). Human microglia cell line (HMC3) was purchased from ATCC (CRL-3304) and grown in EMEM supplemented with 10\% heat-inactivated FBS, $1 \% \mathrm{P} / \mathrm{S}$ solution. Each viral stock was diluted in the appropriate media based on cell types to prepare an infection dose. Infection does, or the MOI was determined from the number of cells to be infected and titer of viral stocks. As higher viral dose had toxic effects on the primary cells, an MOI of 0.1 was used for all studies unless mentioned otherwise. 


\subsubsection{Immunocytochemistry}

Viral infectivity with each viral strain was measured by fluorescent immunolabeling. Briefly, cells were fixed in $4 \%$ paraformaldehyde, permeabilized with $0.1 \%$ Triton $\mathrm{X}-100$, and blocked in $10 \%$ milk/0.1\% goat serum. The fixed cells were immunolabeled using a rabbit anti-ZIKV-E antibody (Cat. GTX133314, Genetex, Irvine, CA, USA). Immunoreactivity was visualized with secondary antibodies from molecular probes (Carlsbad, CA, USA). 4',6-diamidino-2-phenylindole (DAPI) was used to label cell nuclei. The Images were analyzed using an inverted fluorescence microscope with a 560 Axiovision camera (Zeiss, Germany) (Fig. 3C). The purity of the cells was determined immunofluorescent staining for cell-specific marker; glial fibrillary acidic protein (GFAP) for astrocytes (Cat. MAB360, Millipore, Boston, MA, USA), ionized calcium binding adaptor molecules 1 (Iba1) for microglia (Cat. 016-20001, FUJIFILM Wako pure chemical corp, USA) and microtubule-associated protein 2 (MAP2) for neurons (Cat.MAB378, Millipore, Boston, MA, USA).

\subsubsection{Assessment of cell viability}

Glial cell viability was determined by trypan blue dye exclusion method. Briefly, cells were harvested using enzyme-free cell dissociation buffer at the indicated time point. After gentle centrifugation, the resulting cell pellets were re-suspended in respective culture media. An equal volume of $0.40 \%$ trypan blue dye (Cat. 145-0013, Bio-Rad, Hercules, CA, USA) was added to each cell suspension. The total number of viable and nonviable cells were counted using a TC20 automated cell counter (Bio-Rad, Hercules, CA, USA). Cell viability was expressed as percentages of viable cells out of total cells. 
For the assessment of neuronal viability, time-lapse digital images of neurons were recorded using an inverted microscope with an automated computer-controlled stage encoder and environmental chamber $\left(37^{\circ} \mathrm{C}, 95 \%\right.$ humidity, $\left.5 \% \mathrm{CO} 2\right)$ (Zeiss) that allowed repeated tracking of individual neurons per treatment over time. Neuronal death was considered to have occurred upon collapse and fragmentation of the cell body. The viability of neurons was also assessed using a live/dead cell fluorescence assay which combines fluorescent reagents to yield two-color discrimination of the population of live cells indicated by green fluorescence from the dead-cell population indicated by red fluorescence (ScienCell Research Laboratories). Cells were imaged using an inverted fluorescence microscope (Zeiss, Germany) and viable cells were manually quantified and reported as percent of viability.

\subsubsection{Inflammation and growth factor antibody array}

Expression profiles of inflammatory molecules and growth factors were screened by human inflammation antibody array $\mathrm{C} 3$ and human growth factor antibody array $\mathrm{C} 1$ (Ray Biotech, GA, USA), respectively using cell culture supernatant from human astrocytes and microglia infected with different strains of ZIKV. Briefly, antibody array membranes were

incubated with sample for overnight at $4^{0} \mathrm{C}$, followed by biotinylation and streptavidin labeling. Chemiluminescence signals were detected by a ChemiDoc imaging system (BioRad, Hercules, CA, USA).

\subsubsection{Enzyme-linked immune-sorbent assay (ELISA)}

Secretion of interleukin (IL)-6 and -8 , regulated on activation normal $\mathrm{T}$ cell expressed and secreted (RANTES), monocyte chemoattractant protein 1 (MCP-1), IP10/CXCL10 and interferon beta (IFN- $\beta$ ) were measured in culture supernatants of human 
astrocytes infected with three different strains of ZIKV (MR766, R103451 and PRVABC59) for different time points (24, 48,72 and 96 hours) by ELISA (RANTES Cat. DY278-05, MCP-1 Cat. DY279-05, IL-8 Cat. DY208-05, IL-6 Cat. DY206-05 and IFN- $\beta$ Cat. 41410-1, R\&D Systems, Minneapolis, MN, USA). The optical density (O.D.) was read at A450 on a Synergy HTX plate reader (BioTek, Winooski, VT, USA).

\subsubsection{Immunoblotting}

Whole cell lysates were prepared in RIPA buffer (Thermo Scientific, Waltham, MA, USA) supplemented with a mixture of protease and phosphatase inhibitors and separated by SDS-PAGE for immunoblotting. Primary antibodies against ZIKV Envelope protein (Cat. GTX133314, Genetex, Irvine, CA, USA) followed by secondary antibody conjugated to horseradish peroxidase (Millipore, Billerica, MA, USA) were used to label the infected cells. The immunoblots were exposed to SuperSignal West Femto Substrate (Thermo Scientific, Waltham, MA, USA) and visualized using a ChemiDoc imaging system (Bio-Rad, Hercules, CA, USA).

\subsubsection{Statistical analysis}

Results are reported as mean \pm SEM of 3-6 independent experiments each with more than three samples. Data were analyzed using one way, or two-way analysis of variance (ANOVA) followed a Bonferroni post hoc test for multiple comparisons (GraphPad Software, Inc., La Jolla, CA, USA). An alpha level (p-value) of $<0.05$ was considered significant. 


\subsection{Results}

\subsubsection{Viral infectivity differs between the Asian and the African ZIKV strains in}

\section{human astrocytes}

Three spatiotemporally different strains of ZIKV (MR766, R103451, and PRVABC59) were propagated on Vero cells and mosquito cell line (C6/36) followed by quantification using standard plaque assay (Figure 5A-C). The number of virions was expressed as plaque forming units per milliliter (PFU/ml) and was further validated by immunofluorescent labeling of ZIKV Env protein.

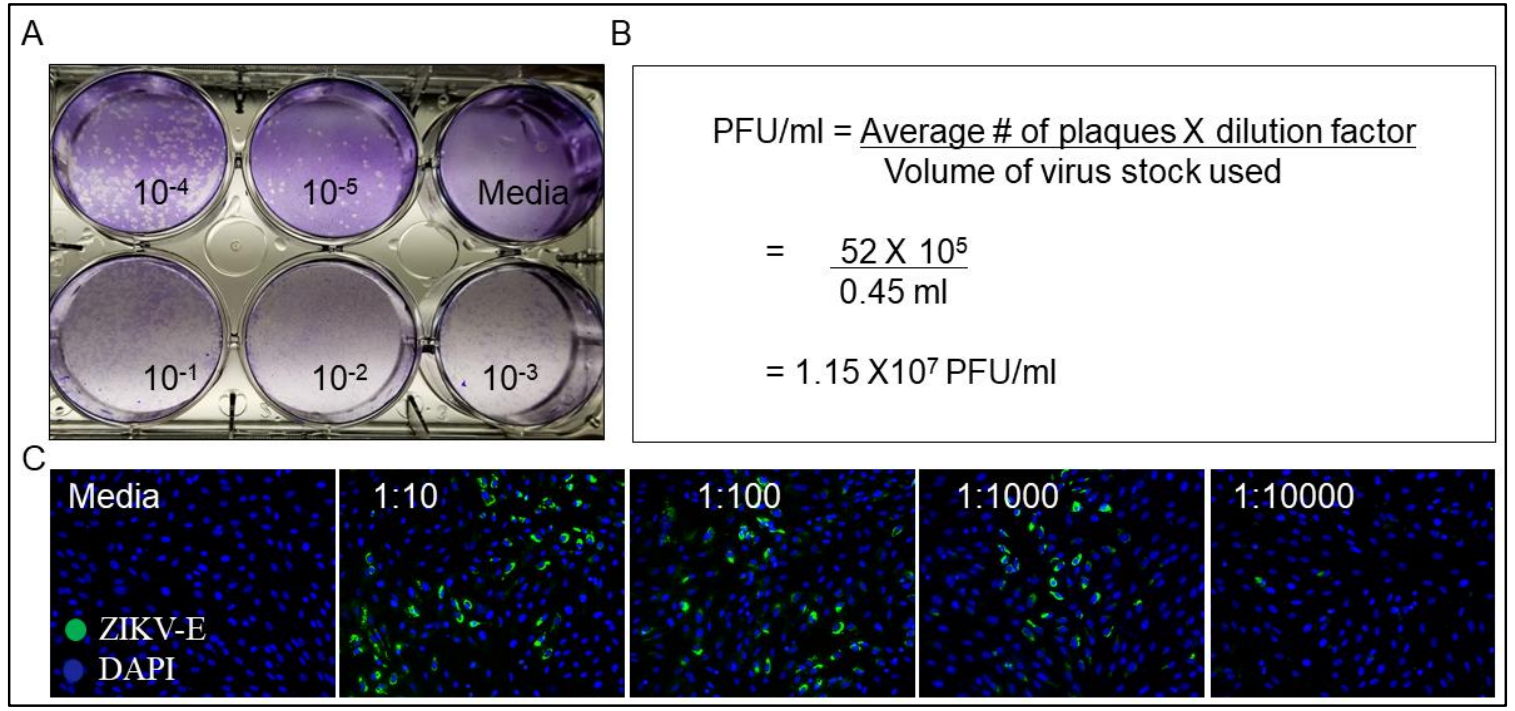

Figure 5. ZIKV propagation and quantification.

ZIKV was propagated in Vero cells and mosquito cell line (C6/36). Viral titer was determined by using a standard plaque assay. Briefly, Vero cells grown to $\geq 90 \%$ confluency were infected with serially diluted viral stock and the cells were overlaid with semisolid media (with $3.2 \%$ carboxymethyl cellulose). After five days of incubation at $37^{\circ} \mathrm{C}$, the cells were washed and stained with $1 \%$ crystal violet to visualize the lysis plaques (A). The number of plaque forming units (PFU) per $\mathrm{ml}$ of the viral stock was calculated using the formula (B). Viral titers were validated by immunofluorescent staining with ZIKV envelope-specific antibody (C).

ZIKV permissiveness in primary human astrocytes was determined by immunofluorescent labeling, RT-PCR and western blot (Figure 6A-D). Human astrocytes 
were infected with either the Asian (R103451 and PRVABC59) or the African (MR766) strains of ZIKV. Twenty-four hours post infection (hpi), infectivity was detected by measuring the abundance of ZIKV envelope protein (Env) by immunofluorescent labeling with anti-ZIKV Env antibody. ZIKV Env was detected in astrocytes infected with each strain of ZIKV although the infectivity was significantly different among the strains with the Asian strains showing higher levels of infectivity. The Asian strains showed the infectivity of about $15-20 \%$ compared to about $10 \%$ infectivity of African strain (Figure 6A and B). Infectivity was confirmed by measuring ZIKV RNA by RT-PCR from the cellular RNA extract, and ZIKV Env protein expression by western blot using cell lysates from infected astrocytes. Both RNA and protein analysis confirmed the immunofluorescence data (Figure 6C and D). Overall, the data showed a higher level of infection with the Asian strains (PRVABC59 and R103451) when compared to the African strain of ZIKV in astrocytes. 

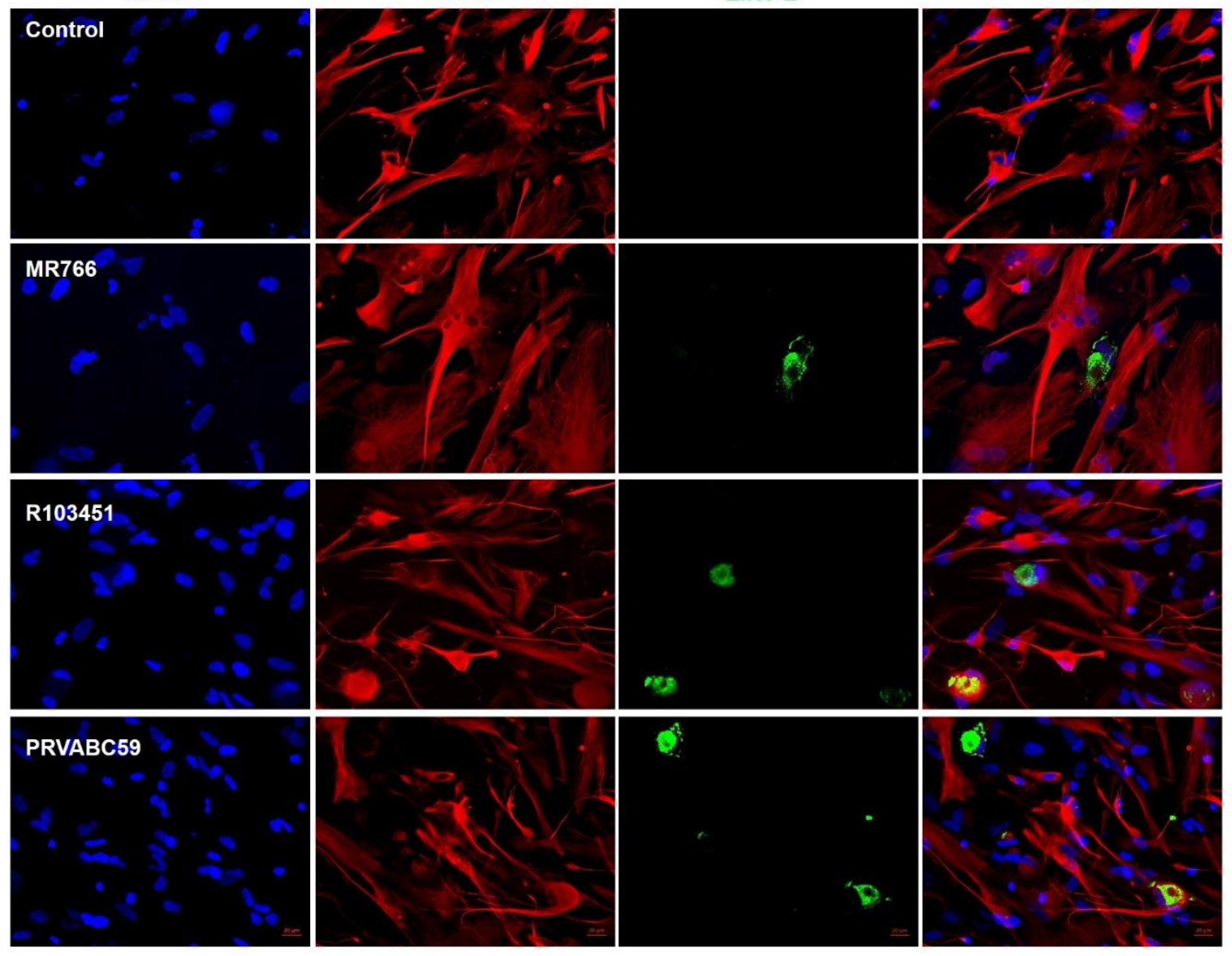

?

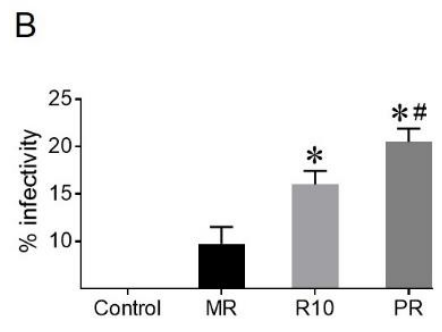

C
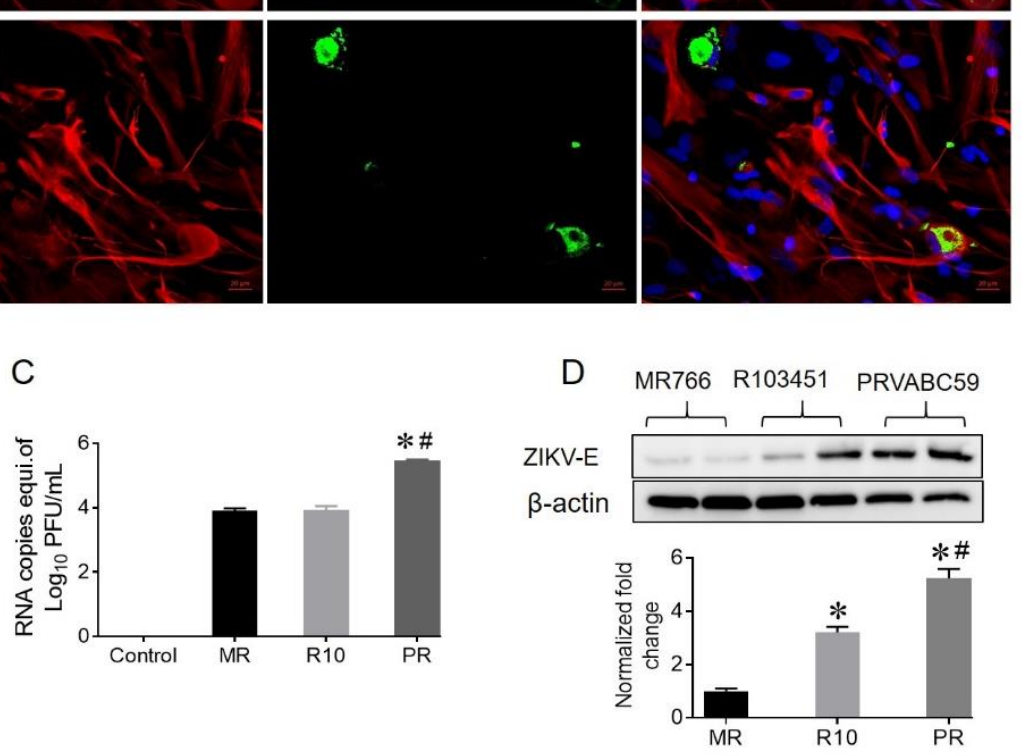

Figure 6. ZIKV infects primary human astrocytes.

ZIKV permissiveness in primary human astrocytes was determined by immunofluorescence staining of infected cells with anti-ZIKV Env antibody (A and B), RT-PCR for viral RNA in infected cells (C) and western blot with the cell lysate collected from the infected cells (D). For each of the experiment, the cells were infected with ZIKV at MOI of 0.1 for 24 hpi. Mock (PBS) infected astrocytes were used as a control. Data are presented as mean \pm SEM from 3 independent experiments and analyzed using one-way ANOVA with Bonferroni post hoc correction. *p $<0.05$ Vs MR and ${ }^{*}$ p $<0.05$ Vs R10. MR: MR766, R10: R103451 and PR: PRVABC59. 


\subsubsection{ZIKV productively infects human astrocytes leading to cell death}

Since we detected ZIKV infection in human astrocytes, we next examined whether the infection is the astrocytes supports viral replication of ZIKV. We measured the number of active viral particles or virions released from the ZIKV-infected astrocytes in the supernatants collected at 24, 48, 72 and 96 hpi by plaque assay. The highest numbers of virions for all three strains of ZIKV were detected at $48 \mathrm{hpi}$, after which the virions were decreased with time (Figure 7A). We detected higher numbers of intracellular viral RNA copies with RT-PCR, when compared to the numbers of viral progenies secreted in the supernatant, detected with plaque assay, suggesting that the number of active viral progeny released in the supernatant might not correlate with the amount of intracellular viral RNA. That was further evidenced by the observation that PRVABC59 strain showed significantly higher RNA titers compared to the other two strains at $24 \mathrm{hpi}$, whereas the differences in virions secretion as measured by plaque assay was observed only at 48 hours (Figure 6C and D). In spite of high infectivity of astrocytes (about $10-25 \%$ with MOI of 0.1 ), we detected a relatively low number of viral progeny $\left(2-4 \log _{10} \mathrm{PFU} / \mathrm{mL}\right)$ suggesting that human astrocytes are efficiently infected but with the limited capacity to produce viral progeny.

To determine cellular toxicity of ZIKV infection, we measured cell viability of human astrocytes after 24, 48, 72 and 96 hpi with different strains of ZIKV. Cell viability was significantly decreased after 48 hpi with all three strains of ZIKV, while the Puerto Rican strain was significantly more toxic to astrocytes in comparison to the African strain MR766 (Figure 7B). 
A

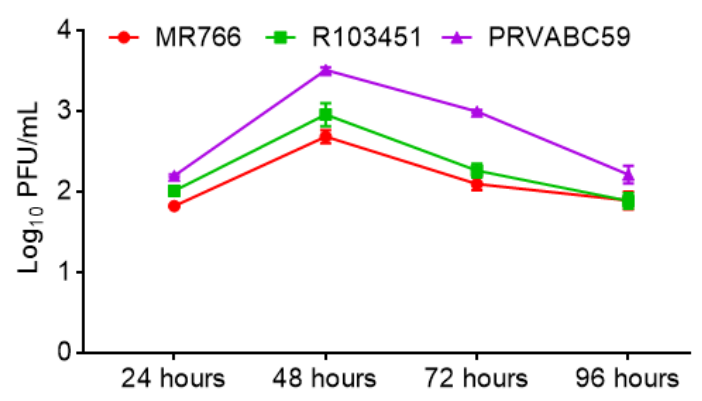

B

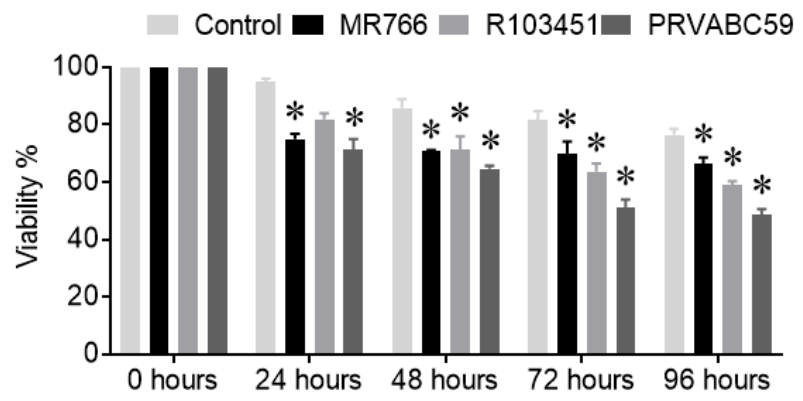

Figure 7. ZIKV replication kinetics and cell viability in primary human astrocytes. Viral release from the cells was measured by plaque assay at indicated time points after infection with MOI of 0.1 of each of the ZIKV strains (A). Cell viability was measured by MTT and trypan blue dye exclusion methods at indicated time points (B). Mock (PBS) infected astrocytes were used as a control. Data are presented as mean \pm SEM from 3 independent experiments and analyzed using two-way ANOVA with Bonferroni post hoc correction. ${ }^{*} \mathrm{p}<0.05$ Vs Control.

\subsubsection{Viral infectivity of ZIKV in human microglia also differs among the strains}

Since microglia are also important glial cells and resident macrophages in the brain, we measured the infectivity, viral kinetics and cell viability of ZIKV infected human microglia. Human microglia showed similar levels of ZIKV infectivity as astrocytes (Figure 8A). Unlike astrocytes, the Honduran strain of ZIKV (R103451) showed the lowest infectivity in microglia. We observed similar trends of viral kinetics results in microglia except for those infected with MR766 strain, where the highest number of virions was reported at 72 hpi (Figure 8B). Based on the immunofluorescent and plaque assay data, microglia are less permissive and productive to Honduran strain compared to Uganda and Puerto-Rican strains of ZIKV. A decrease in viability was also noted in microglia infected with Asian and African strains of ZIKV and was commensurate with the viral infectivity and virions released (Figure 8C). 

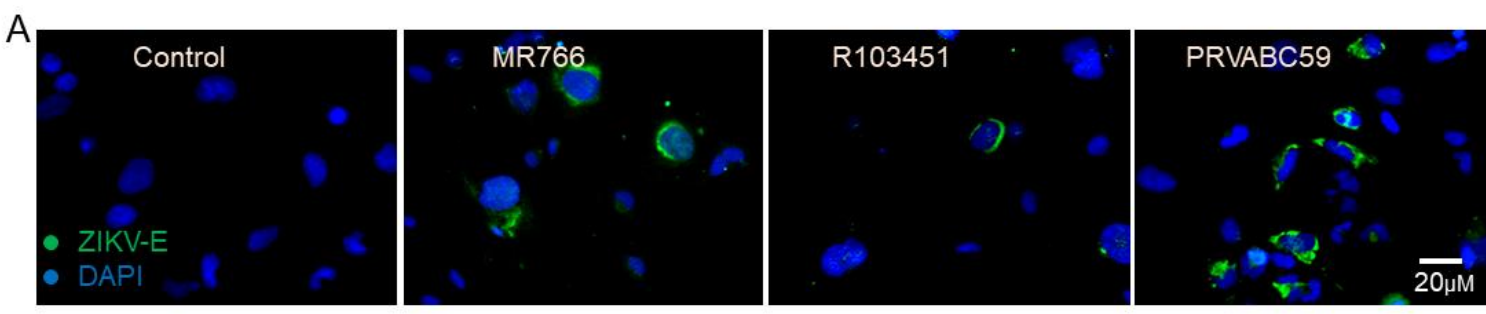

B
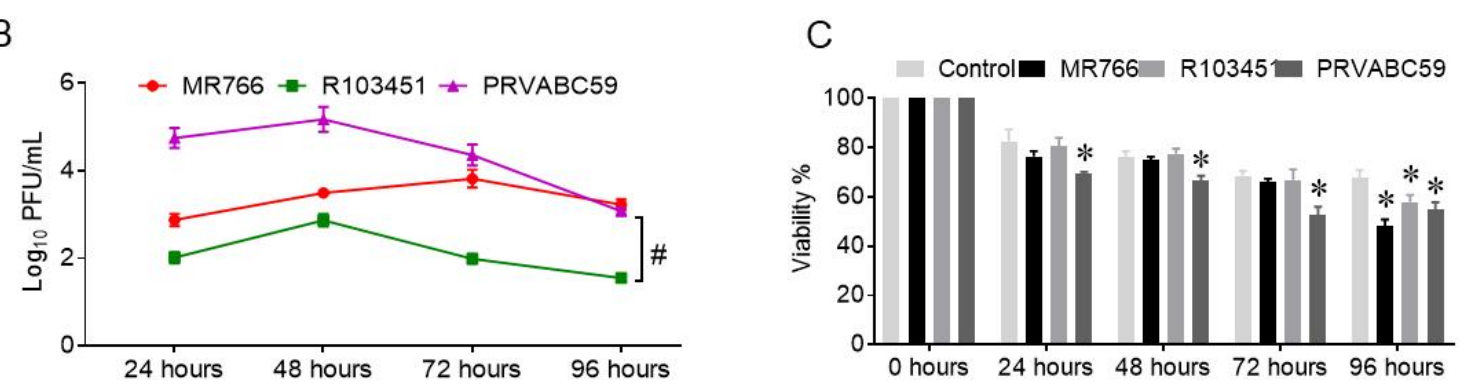

Figure 8. ZIKV infects primary human microglia.

ZIKV permissiveness in human microglia was determined by immunofluorescence staining of infected cells with anti-ZIKV Env antibody (A). Viral release from the cells was measured by plaque assay at indicated time points after infection with MOI of 0.1 of each of the ZIKV strains (B). Cell viability was measured by MTT and trypan blue dye exclusion methods at indicated time points (C). Mock (PBS) infected astrocytes were used as a control. Data are presented as mean \pm SEM from 3 independent experiments and analyzed using two-way ANOVA with Bonferroni post hoc correction. ${ }^{*} \mathrm{p}<0.05 \mathrm{Vs}$ control and ${ }^{\#} \mathrm{p}<0.05$ Vs MR766.

\subsubsection{ZIKV infects human primary neurons and induces cell death}

Most of the current studies focus on the ZIKV infection in neuroprogenitor cells and undifferentiated neurons. Much less is known about the ZIKV infection in the mature differentiated neurons. Therefore, we infected mature differentiated human primary neurons with different strains of ZIKV to examine the infectivity and the toxicity. We showed that all three strains of ZIKV are capable of directly infecting human neurons (Figure 9A). Neuronal viability was measured using time-lapse image analysis and fluorescent live/dead assay (Figure 9B-D). Time-lapse image analysis of infected neurons showed the Honduras strain (R103451) induced the highest neuronal death (around 35\% at 
48 hpi) followed by Puerto-Rican strain (PRVABC59) (Figure 9B). The live-dead assay showed significantly higher percentages (about 60\%) of neuronal death with Asian strains of ZIKV compared to neuronal death with African strain of ZIKV, which was about 30\% (Figure 9D).
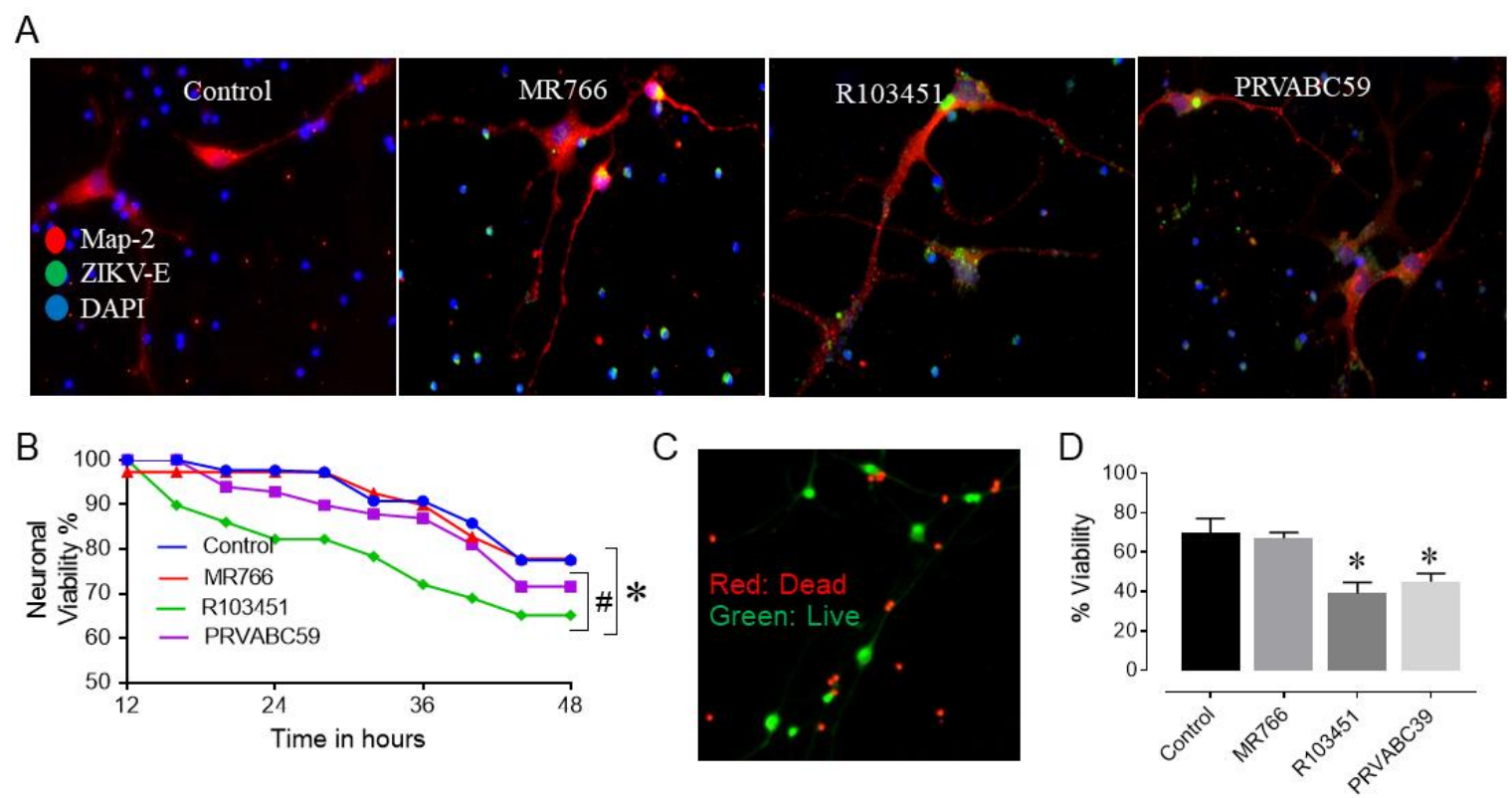

Figure 9. ZIKV infects primary human neuron inducing cell death.

ZIKV permissiveness in human primary neurons was determined by immunofluorescence staining of infected cells with anti-ZIKV Env antibody and MAP-2, a neuronal marker (A). Cell viability was measured by analyzing the time-lapse image of the individual neurons taken at multiple time points (B) and was further confirmed by live dead fluorescent staining of neurons at the end of the time-lapse experiment (C and D). Mock (PBS) infected astrocytes were used as a control. Data are presented as mean \pm SEM from 3 independent experiments and analyzed using one-way ANOVA with Bonferroni post hoc correction. *p $<0.05$ Vs Control, \#p < 0.05 Vs MR766.

Overall, the rate of infectivity among each viral strain of ZIKV correlated with cell death in human astrocytes, microglia, and neurons. Puerto Rican strain (PRVABC59) of ZIKV was more infectious and replicated more vigorously in glial cells which correlated with more cell death, while Honduran strain showed more toxicity in neurons. 


\subsubsection{Asian strains of ZIKV induce higher levels of inflammatory molecules in human glial cells}

As glial cells are the principal cell types involved in the release of neuro-inflammatory molecules, a key marker of viral pathology in the brain, we examined changes in the secretion of inflammatory molecules by astrocytes infected with different strains of ZIKV (MOI of 0.1). Human inflammatory antibody array was performed using the supernatants collected from the ZIKV infected astrocytes at 48 hpi (Figure 10A). Different inflammatory molecules including IL-6, IL-1 $\alpha$, IL-4, IP-10, RANTES and transforming growth factor $\beta 1$ (TGF- $\beta 1$ ) were induced in human astrocytes by all three strains of ZIKV. The Puerto Rican strain (PRVABC59) had a more robust effect on the release of inflammatory factors (Figure 10B).
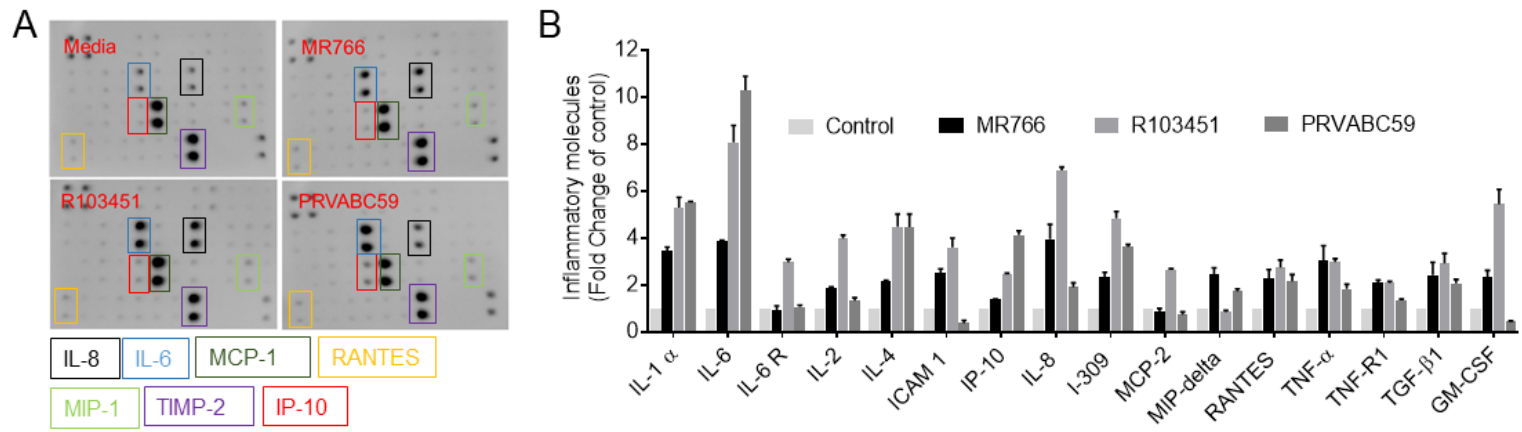

Figure 10. ZIKV significantly induces secretion of inflammatory molecules in human astrocytes.

Secretion of various inflammatory molecules by astrocytes infected with different strains of ZIKV (MOI of 0.1) was measured by using Human inflammatory antibody array (A). The inflammatory molecules that were highly expressed in human astrocytes and were also induced by ZIKV are presented in figure (B). Mock (PBS) infected astrocytes were used as a control. Data are presented as mean \pm SEM from 3 independent experiments and analyzed using one-way ANOVA with Bonferroni post hoc correction. ${ }^{*} \mathrm{p}<0.05 \mathrm{Vs}$ Control. 

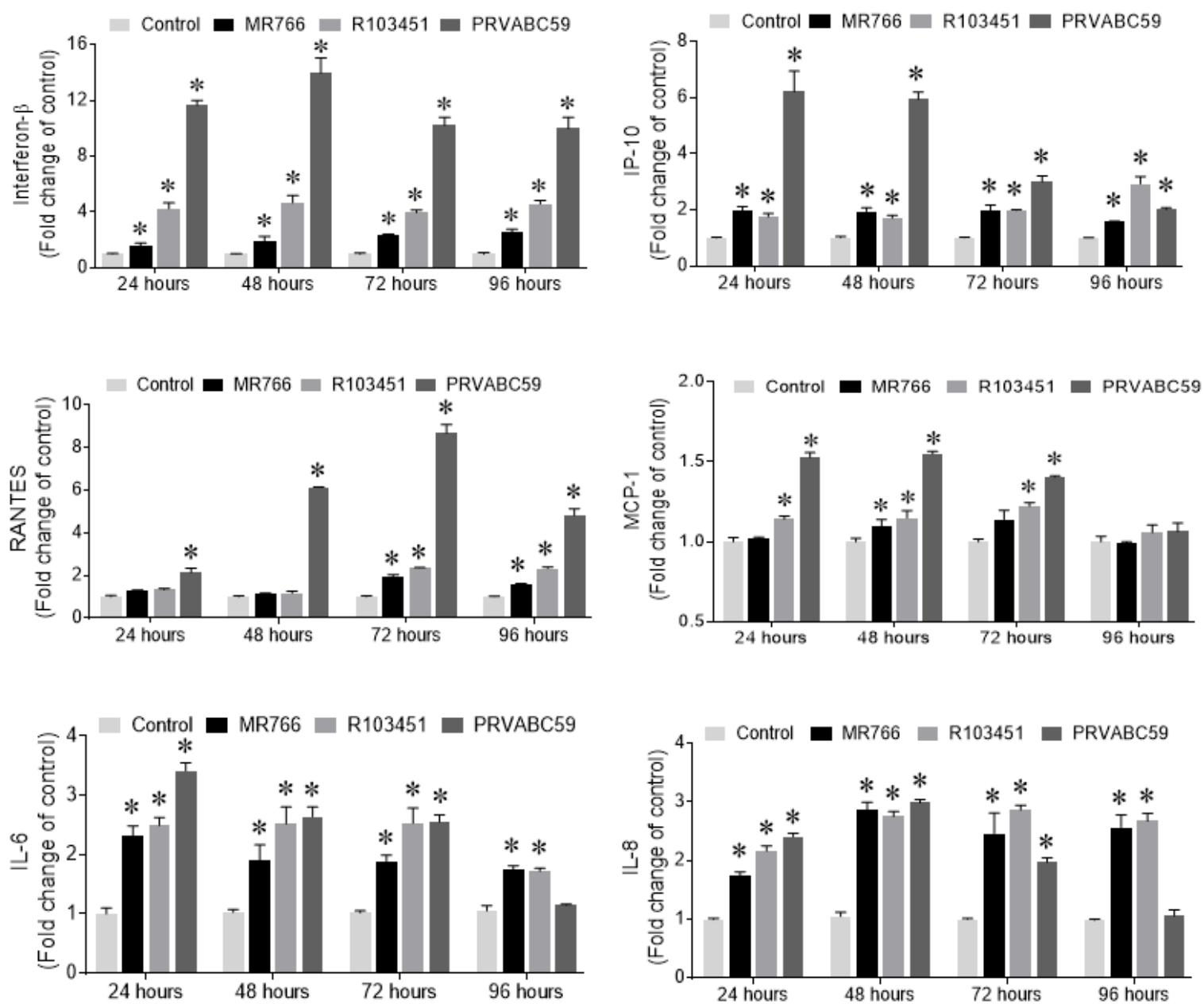

Figure 11. ZIKV induces key antiviral and inflammatory molecules in human astrocytes.

ELISA was performed to quantify the secretion of IFN- $\beta$, IP-10, RANTES, IL- 6 and IL-8 from the supernatants collected from the infected astrocytes at indicated time points. The data are expressed as the fold change of control (uninfected astrocytes). Mock (PBS) infected astrocytes were used as a control. Data are presented as mean \pm SEM from 3 independent experiments and analyzed using two-way ANOVA with Bonferroni post hoc correction. *p $<0.05$ Vs Control.

We confirmed our results using individual ELISA for MCP-1, RANTES, IL-8, IP10, and IL-6. Both the Asian and African strains of ZIKV induced the secretion of MCP1, RANTES, IP-10, IL-8 and IL-6 (Figure 11). Interestingly, the Puerto Rican strain (PRVABC59) had the largest effect causing about 8-fold increase in RANTES, a 6-fold increase in IP-10, a 1.5-fold increase in MCP-1 and a 3.5-fold increase in IL-8 and IL-6 
when compared to uninfected control. Except for IL-8, peak levels of the inflammatory molecules were detected at 48 or $72 \mathrm{hpi}$, which correlated with the detected viral titers (Figure 5D). The kinetics of cytokines/chemokines secretion showed that the PRVABC59 strain caused a rapid cytokine and chemokine response which decreases with time. However, the MR766 strain induced a slower, persistent cytokine response. Since type 1 IFN is considered an indicator of early antiviral response by host cells to restrict virus spread [15], we measured type 1 IFN levels (IFN- $\alpha$ and IFN- $\beta$ ) using ELISA and detected secretion of IFN- $\beta$ in human astrocytes infected with all three ZIKV strains (Figure 11), whereas IFN- $\alpha$ was undetectable (data not shown). Although all three strains showed a significant increase in the secretion of IFN- $\beta$ levels, the Puerto Rican strain (PRVABC59) induced more than a 12-fold increase in secretion with a peak reaching at 48 hpi (Figure 11), suggesting a more rigorous antiviral response. The sustained IFN- $\beta$ response by human astrocytes against the PRVABC59 strain of ZIKV throughout 96 hours' correlates with the suppression of viral titers.

In human microglia, the inflammatory antibody array shows increased secretion of RANTES, IP-10, Il-6, MIP-1 $\alpha$, and MIP-1B with the PRVABC59 strain, but minor twitches were observed with other two strains of ZIKV (Figure 12A). We further confirmed the semi-quantitative data from the membrane-based assay with ELISA (Figure 12B-D). The ELISA data shows that IP-10 was significantly induced by MR766 and PRVABC59 strains of ZIKV up to $72 \mathrm{hpi}$, whereas, RANTES was typically induced by PRVABC59 strain of ZIKV up to $96 \mathrm{hpi}$. There was significant upregulation in IL-6 secretion by all three strains of ZIKV up to 72 hpi. The lowest infectivity of Honduran 
strain of ZIKV correlates with the minimal effect of the virus on the secretion of these inflammatory molecules.
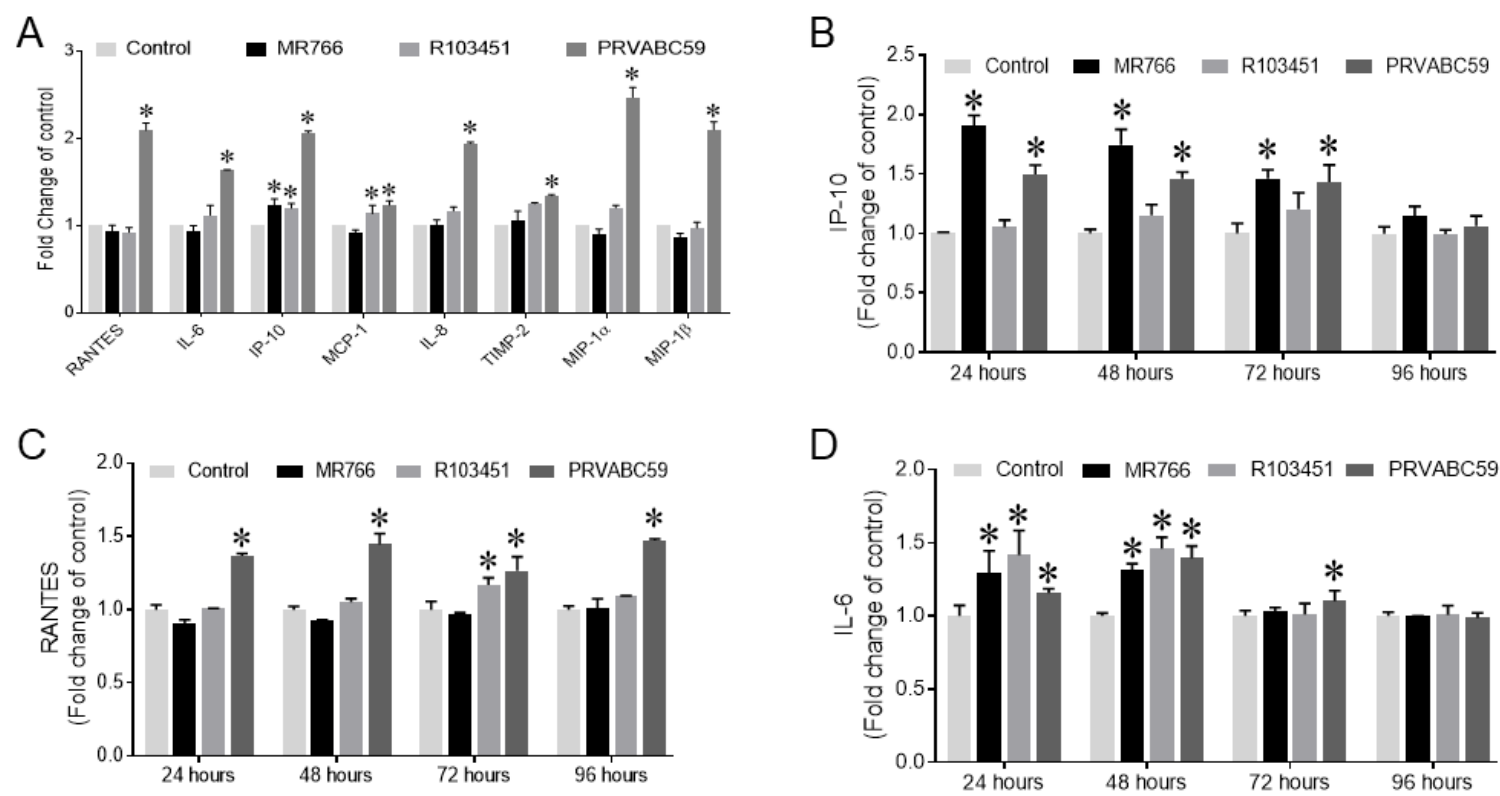

Figure 12. ZIKV induces key antiviral and inflammatory molecules in human microglia.

Human inflammatory antibody array was performed to screen the inflammatory molecules involved in ZIKV infection in microglia (A) ELISA was performed to quantify the secretion of IP-10, RANTES, IL-6 and IL-8 from the supernatants collected from the infected astrocytes at indicated time points. The data are expressed as the fold change of control (uninfected microglia). Mock (PBS) infected astrocytes were used as a control. Data are presented as mean \pm SEM from 3 independent experiments and analyzed using two-way ANOVA with Bonferroni post hoc correction. *p <0.05 Vs Control.

\subsubsection{ZIKV caused dysregulation of growth factor expression in human astrocytes}

Since ZIKV has been linked to growth retardation and microcephaly which might be caused by an alteration in the expression or release of growth factors, we analyzed changes in human growth factors using an antibody array. Cell culture supernatants from human astrocytes infected with the three different strains of ZIKV were analyzed. With the exception of insulin-like growth factor binding protein-6 (IGFBP-6) which showed a 2fold increase in expression, minor increases in the release of the growth factors monocyte- 
colony stimulating factor (M-CSF), epidermal growth factor (EGF), basic fibroblast growth factor (bFGF) and decreases in insulin-like growth factor-2 (IGF-2), insulin-like growth factor binding protein 2 (IGFBP-2) and platelet-derived growth factor-AA (PDGFAA) (Figure 13) were detected in astrocytes infected with the Puerto Rican strain (PRVABC59). Although we observed slight changes in the expression levels of several other growth factors with both the Asian and African strains, these were not significant (data not shown).

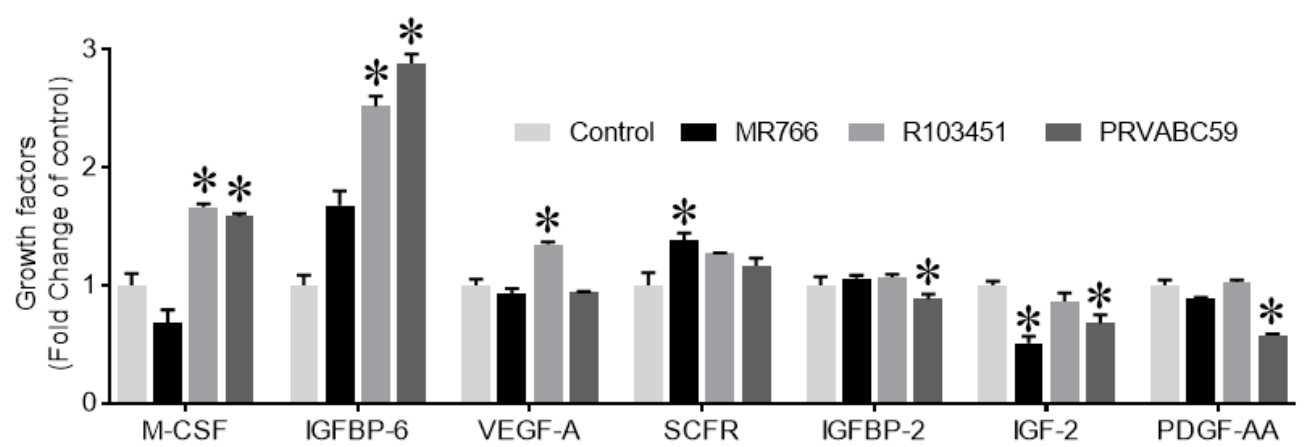

Figure 13. ZIKV induces minor changes in expression of growth factor in human astrocytes as measured by using Human growth factor antibody array.

Mock (PBS) infected astrocytes were used as a control. Data are presented as mean \pm SEM from $\geq 3$ independent experiments and analyzed using one-way ANOVA with Bonferroni post hoc correction. *p $<0.05$ Vs Control.

\subsection{Discussion}

The association of recent ZIKV outbreaks with congenital birth defects and neurological disorders has brought great attention to this global health problem. There is a dire need for the development of a safe and effective vaccine against this neurotropic virus. The mechanisms behind the neuropathologies mediated by ZIKV are still unclear. More severe neurological consequences of ZIKV have been reported with the Asian lineage of ZIKV, indicating differential ability between the Asian and the African lineage of ZIKV to infect brain cells and develop neurological complications. Our study aimed to describe 
the comparative infectivity of three different strains of ZIKV and explore the profile of neuro-inflammatory molecules induced following the infection. Based on the isolation history, genomic sequence analysis, phylogenetic analysis and geographical distribution of the virus, we selected three viral strains representing both Asian and African lineage of the virus.

We reported that the Asian lineage of ZIKV, specifically the Puerto Rican strain (PRVABC59) showed higher infection which correlated with higher levels of proinflammatory molecules secretion and increased cell death in glial cells. A recent study has reported that Asian (ZIKV-Br) and African strains (MR766) of ZIKV are almost equally infective to human brain cortical astrocytes inducing secretion of IP-10, IL-6, IL-8, and RANTES [16]. Contrasting to our findings, Huang et al. observed higher infectivity with African strain (MR766) than the Asian strain (PRVABBC59) in human fetal astrocytes [17]. The discrepancy among these studies might be associated with the viral strains, passage numbers, and sources of astrocytes, MOI of virus used, and other factors. Nevertheless, all of the studies conclude that ZIKV strains differentially infect astrocytes and induce antiviral inflammatory responses with typically higher levels of chemokines. Glial cells have a primary role in the innate immunity in the brain by recognition of pathogens and their clearance [18]. Here, we focused on astrocytes due to their abundance in the brain, their direct linkage with neurons and their role in synaptic support, axonal guidance, and involvement in the BBB [19]. Herein, we reported a significant increase in the inflammatory molecules, RANTES, MCP-1, IP-10, IL-8 and IL-6 with ZIKV infection in glial cells. CXCL10/IP-10, an important biomarker of severity in many infectious diseases and anti-flaviviral response in the CNS was previously reported to be induced by 
ZIKV and other flaviviruses [20-23]. Notably, IP-10 is strongly implicated in GBS, indicating that elevated levels of IP-10 might mediate ZIKV-mediated neuronal injury. In this study, the Asian strains of ZIKV, specifically the Puerto Rican strain (PRVABBC59), which is more likely to cause neurodegenerative disorders, showed a very high level (up to 6-fold) of IP-10 secretion by astrocytes. The pro-inflammatory molecules; IL-6, IL-8, RANTES, and MCP-1, which recruit and activate other cell types to the infection site and amplify the inflammatory cascades, were augmented by all three strains of ZIKV in astrocytes. ZIKV induced IFN- $\beta$ in general; however, the secretion levels were straindependent with the Puerto Rican strain inducing around a 14-fold increase. Although inflammatory molecules and IFNs are secreted as a host defense mechanism to attenuate viral burden, the high levels of inflammatory molecules secreted by brain cells may disrupt the BBB and facilitate further spread of the virus in CNS [21, 24]. Cytokine kinetics in our study mimics the kinetics reported in clinical cases of ZIKV where RANTES, IL-6, IP10, and IFN- $\beta$ were elevated both in acute and convalescent phases of ZIKV infection, albeit higher levels were detected during the acute phase. On the other hand, a higher level of IL8 was detected during the convalescent phase than the acute phase of ZIKV infection. Moreover, elevations in chemokines were more pronounced than in cytokines [25]. In line with our study, different kinetics of anti-viral responses in astrocytes infected with Asian (H/PF/2013) and African strain (HD78788) of ZIKV has been reported by Hamel et.al. A more rapid antiviral response, as shown by increased expression of several inflammatory molecules including IP-10, RANTES, IL-6, and IFN- $\beta$ within 6 hpi, was induced with Asian strains when compared to the African strain which induced delayed response [26]. Although the changes were not significant, we observed minor changes in the growth 
factors, M-SCF, IGFBP-6, IGFBP-2, IGF-2, and PDGF-AA in ZIKV infected astrocytes, which may contribute to ZIKV associated neurodevelopmental disorders.

In our study, we reported significantly more cell death mediated by ZIKV compared mock-infected cells. The secretion of exaggerated levels of inflammatory molecules by glial cells might have contributed to cell death. Though type 1 IFN might be the classical antiviral response, persistent higher levels of secreted inflammatory cytokines and chemokines induce a pattern of acute or chronic inflammation potentially leading to neurotoxicity. Some of the cytokines are also involved in the activation of the death receptor facilitating the apoptosis mediated cell death besides the formation of the inflammasome. Combined with the studies showing activation of apoptosis by ZIKV, our data indicate that ZIKV induced inflammatory molecules secretion may lead to apoptosis [27]. Cell death, in turn, may provoke an inflammatory response [28].

In summary, we report differences in infectivity rate and the secretion of inflammatory molecules in human glial cells infected with three different spatiotemporal strains of ZIKV. Excessive and persistent inflammatory responses potentially lead to ZIKV-related cell death. The differences in infectivity levels, inflammatory responses, and cellular toxicity may account for differential pathology in the viral strains and clinical manifestations in affected populations.

\section{References}

[1] de Araújo TVB, Rodrigues LC, de Alencar Ximenes RA, de Barros MirandaFilho D, Montarroyos UR, de Melo APL, et al. Association between Zika virus infection and microcephaly in Brazil, January to May, 2016: preliminary report of a case-control study. The Lancet Infectious Diseases. 2016;16(12):1356-63. 
[2] Parra B, Lizarazo J, Jimenez-Arango JA, Zea-Vera AF, Gonzalez-Manrique G, Vargas J, et al. Guillain-Barre Syndrome Associated with Zika Virus Infection in Colombia. N Engl J Med. 2016;375(16):1513-23.

[3] Simonin Y, Loustalot F, Desmetz C, Foulongne V, Constant O, Fournier-Wirth C, et al. Zika Virus Strains Potentially Display Different Infectious Profiles in Human Neural Cells. EBioMedicine. 2016;12:161-9.

[4] Liang Q, Luo Z, Zeng J, Chen W, Foo S-S, Lee S-A, et al. Zika Virus NS4A and NS4B Proteins Deregulate Akt-mTOR Signaling in Human Fetal Neural Stem Cells to Inhibit Neurogenesis and Induce Autophagy. Cell Stem Cell. 19(5):66371.

[5] van den Pol AN, Mao G, Yang Y, Ornaghi S, Davis JN. Zika Virus Targeting in the Developing Brain. The Journal of Neuroscience. 2017;37(8):2161.

[6] Miner Jonathan J, Diamond Michael S. Understanding How Zika Virus Enters and Infects Neural Target Cells. Cell Stem Cell. 2016;18(5):559-60.

[7] Liu B, Hong J-S. Role of Microglia in Inflammation-Mediated Neurodegenerative Diseases: Mechanisms and Strategies for Therapeutic Intervention. Journal of Pharmacology and Experimental Therapeutics. 2003;304(1):1-7.

[8] Shao Q, Herrlinger S, Yang SL, Lai F, Moore JM, Brindley MA, et al. Zika virus infection disrupts neurovascular development and results in postnatal microcephaly with brain damage. Development (Cambridge, England). 2016;143(22):4127-36.

[9] Faye O, Freire CC, Iamarino A, Faye O, de Oliveira JV, Diallo M, et al. Molecular evolution of Zika virus during its emergence in the 20(th) century. PLoS Negl Trop Dis. 2014;8(1):e2636.

[10] Tripathi S, Balasubramaniam VRMT, Brown JA, Mena I, Grant A, Bardina SV, et al. A novel Zika virus mouse model reveals strain specific differences in virus pathogenesis and host inflammatory immune responses. PLOS Pathogens. 2017;13(3):e1006258.

[11] Lazear Helen M, Govero J, Smith Amber M, Platt Derek J, Fernandez E, Miner Jonathan J, et al. A Mouse Model of Zika Virus Pathogenesis. Cell Host \& Microbe. 19(5):720-30.

[12] Smith DR, Sprague TR, Hollidge BS, Valdez SM, Padilla SL, Bellanca SA, et al. African and Asian Zika Virus Isolates Display Phenotypic Differences Both In Vitro and In Vivo. Am J Trop Med Hyg. 2017. 
[13] Anfasa F, Siegers JY, Kroeg Mvd, Mumtaz N, Raj VS, Vrij FMSd, et al. Phenotypic Differences between Asian and African Lineage Zika Viruses in Human Neural Progenitor Cells. 2017.

[14] Agbulos DS, Barelli L, Giordano BV, Hunter FF. Zika Virus: Quantification, Propagation, Detection, and Storage. Current protocols in microbiology. 2016;43:15d.4.1-d.4.6.

[15] Boasso A. Type I Interferon at the Interface of Antiviral Immunity and Immune Regulation: The Curious Case of HIV-1. Scientifica. 2013;2013:20.

[16] Stefanik M, Formanova P, Bily T, Vancova M, Eyer L, Palus M, et al. Characterisation of Zika virus infection in primary human astrocytes. BMC Neuroscience. 2018;19(1):5.

[17] Huang Y, Li Y, Zhang H, Zhao R, Jing R, Xu Y, et al. Zika virus propagation and release in human fetal astrocytes can be suppressed by neutral sphingomyelinase2 inhibitor GW4869. Cell Discovery. 2018;4(1):19.

[18] Bailey SL, Department of Microbiology-Immunology and The Interdepartmental Immunobiology Center FSoM, Northwestern University, Chicago, IL 60611, review Acett, Carpentier PA, Department of Microbiology-Immunology and The Interdepartmental Immunobiology Center FSoM, Northwestern University, Chicago, IL 60611, review Acett, et al. Innate and Adaptive Immune Responses of the Central Nervous System. Critical Reviews ${ }^{\mathrm{TM}}$ in Immunology. 2006;26(2).

[19] Blackburn D, Sargsyan S, Monk PN, Shaw PJ. Astrocyte function and role in motor neuron disease: a future therapeutic target? Glia. 2009;57(12):1251-64.

[20] Ferreira RAX, de Oliveira SA, Gandini M, Ferreira LdC, Correa G, Abiraude FM, et al. Circulating cytokines and chemokines associated with plasma leakage and hepatic dysfunction in Brazilian children with dengue fever. Acta Tropica. 2015;149:138-47.

[21] Naveca FG, Pontes G, Chang A, Silva GAVd, Nascimento VAd, Monteiro DCdS, et al. Analysis of the immunological biomarker profile during acute Zika virus infection reveals the overexpression of CXCL10, a chemokine already linked to neuronal damage. bioRxiv. 2017.

[22] Marle Gv, Antony J, Ostermann H, Dunham C, Hunt T, Halliday W, et al. West Nile Virus-Induced Neuroinflammation: Glial Infection and Capsid ProteinMediated Neurovirulence. Journal of virology. 2007. 
[23] Liu Y, Chen L, Zou Z, Zhu B, Hu Z, Zeng P, et al. Hepatitis C virus infection induces elevation of CXCL10 in human brain microvascular endothelial cells. J Med Virol. 2016;88(9):1596-603.

[24] Wang T, Town T, Alexopoulou L, Anderson JF, Fikrig E, Flavell RA. Toll-like receptor 3 mediates West Nile virus entry into the brain causing lethal encephalitis. Nature Medicine. 2004;10(12):1366.

[25] Tappe D, Pérez-Girón JV, Zammarchi L, Rissland J, Ferreira DF, Jaenisch T, et al. Cytokine kinetics of Zika virus-infected patients from acute to reconvalescent phase. Medical Microbiology and Immunology. 2016;205:269-73.

[26] Hamel R, Ferraris P, Wichit S, Diop F, Talignani L, Pompon J, et al. African and Asian Zika virus strains differentially induce early antiviral responses in primary human astrocytes. Infection, Genetics and Evolution. 2017;49:134-7.

[27] Azevedo RSS, de Sousa JR, Araujo MTF, Martins Filho AJ, de Alcantara BN, Araujo FMC, et al. In situ immune response and mechanisms of cell damage in central nervous system of fatal cases microcephaly by Zika virus. Scientific reports. $2018 ; 8(1): 1-$.

[28] Rock KL, Kono H. The inflammatory response to cell death. Annual review of pathology. 2008;3:99-126. 


\section{CHAPTER 3. MECHANISM OF ZIKA VIRUS ENTRY INTO HUMAN PRIMARY}

\section{ASTROCYTES}

\subsection{Introduction}

ZIKV, similar to other members of the flavivirus, enters the cell presumably by receptor-mediated endocytosis $[1,2]$. The molecular mechanisms of viral entry into brain cells and the associated pathology are still poorly understood. Discovery of the mechanism of entry and associated factors is one of the most important aspects of viral pathogenesis and cellular tropism. Current studies suggest that Tyro3 Axl MER (TAM) family of receptors are shown to be the primary entry factors for ZIKV in various cell types $[2,3]$.

Out of the putative ZIKV receptors, Axl is the most studied receptor in regards to ZIKV infection. Axl is a member of the TAM family of receptors that was originally cloned from cancer cells [4]. The receptor has two ligands including the growth arrest-specific-6 (Gas6) and the protein S [5]. Axl is involved in the innate immune response as well as other cellular mechanisms including proliferation, migration, and aggregation in different cell types [6, 7].

Exposure to neutralizing antibody against Axl or by gene silencing using small interfering RNA targeting the Axl gene led to a decrease in viral replication and infection, suggesting a role of Axl as a putative ZIKV entry receptor in human fibroblast cells [8]. Human neural progenitor cells, microglia, and astrocytes isolated from developing brain express Axl receptors. A previous study has shown that inhibition of Axl by synthetic decoy receptor (MYD1) and Axl kinase inhibitor (R428) resulted in the inhibition of ZIKV infection in the glial cells [2]. However, others have reported that silencing the Axl gene was unable to inhibit viral entry but rather facilitates the upregulation of type 1 interferon 
signaling, indicating that Axl promotes ZIKV infection in glial cells by antagonizing type I interferon (IFN) signaling. It is still unclear whether ZIKV entry and subsequent replication are solely dependent on the Axl receptor [9-11].

The controversial reports regarding the role of TAM (Axl) receptor in ZIKV infection in brain cells necessitate further investigation using more than one spatiotemporal ZIKV strains and primary cells because the cell lines might not express the receptors and may behave differently than the primary cells $[10,11]$. Therefore, effective contributions of Axl and other members of TAM receptors in ZIKV entry and pathogenesis need to be investigated using primary brain cells. The objective of this study was to examine the role of TAM receptors, including Axl in ZIKV infection using primary human astrocytes, the major cell types in the brain. Herein we used genetic and pharmacological approaches to downregulate the receptors and report that Axl receptor but not the Tyro3 receptor is involved in ZIKV infection and replication in human astrocytes and associated antiviral response.

\subsection{Methodology}

\subsubsection{Cell cultures, treatment, and ZIKV infection}

Human primary astrocytes (Cat. 1800, ScienCell, Carlsbad, CA, USA) were grown and maintained in astrocyte medium supplemented with $2 \%$ FBS, $1 \%$ astrocyte growth supplement (AGS) and 1\% antibiotic/antimycotic solution (ScienCell, Carlsbad, CA, USA). Each viral stock was diluted in the appropriate media based on cell types. Infection dose or the MOI was determined from the number of cells to be infected and titer of viral stocks. As higher viral dose had toxic effects on the primary cells, an MOI of 0.1 was used for all studies unless mentioned otherwise. 


\subsubsection{Immunoblotting}

Whole cell lysates were prepared in RIPA buffer (Thermo Scientific, Waltham, MA, USA) supplemented with a mixture of protease and phosphatase inhibitors and separated by SDS-PAGE for immunoblotting. Primary antibodies against ZIKV Envelope protein (Cat. GTX133314, Genetex, Irvine, CA, USA) followed by secondary antibody conjugated to horseradish peroxidase (Millipore, Billerica, MA, USA) was used to label the infected cells. The immunoblots were exposed to SuperSignal West Femto Substrate (Thermo Scientific, Waltham, MA, USA) and visualized using a ChemiDoc imaging system (Bio-Rad, Hercules, CA, USA).

\subsubsection{RNA interference: silencing of the Axl receptor}

Small interfering RNA (siRNA) Axl (Cat. sc-29769) was purchased from Santa Cruz Biotechnology (Santa Cruz, CA, USA). Human primary astrocytes were transfected with siRNA against Axl for 24 - 48 hours using Lipofectamine 2000 (Invitrogen, Carlsbad, CA, USA) or Fugene HD (Promega, Madison, WI, USA) for Axl knock down. siRNA and transfection reagent (ratio, 1:2) were pre-incubated for $20 \mathrm{~min}$ in OptiMEM medium before addition to the seeded cells. Knockdown of targets was confirmed in cell lysates by western blot for the protein expression of Axl receptor using a primary antibody against Axl (Cat. sc-166269, Santa Cruz Biotech, USA).

\subsubsection{Inhibition of Axl and Tyro3 by small molecule inhibitors}

Human primary astrocytes were grown to 80-90\% confluency in astrocytes-specific media as described in chapter 2. Half an hour prior to ZIKV infection, the cells were treated with increasing concentrations $(0.1,0.5$ and $1 \mu \mathrm{M})$ of either small molecule inhibitor of Axl (R428, Cat.S2841) or Tyro3 inhibitor (BMS777607, cat. S1561, Selleck, USA). The 
concentration of the inhibitors was selected based on the previous literature and their inhibitory concentration 50 (IC-50). With continuous exposure of the cells to the inhibitor, cells were infected with ZIKV as mentioned previously.

\subsubsection{Statistical analysis}

Results are reported as mean \pm SEM of 3-6 independent experiments each with more than three samples. Data were analyzed using analysis of variance (ANOVA) followed by appropriate post hoc test for multiple comparisons (GraphPad Software, Inc., La Jolla, CA, USA). An alpha level (p-value) of $<0.05$ was considered significant.

\subsection{Results}

\subsubsection{TAM receptors and their ligands are expressed in human astrocytes.}

To determine a potential mode of viral entry in human glial cells, we investigated the expression of putative host receptors utilized by ZIKV, as reported by others $[2,3]$. Protein expression of the receptors was assessed by immunoblotting of the cell lysate with or without ZIKV infection. Members of the TAM receptors, Tyro3, Axl, and MERTK were detected in human astrocytes, but surprisingly infection with any of the three ZIKV strains had no effect on protein expression levels of Axl, although a slight increase in the expression of Tyro3 was observed with both Asian and African strains (Figure 14). We

also investigated the expression of growth arrest- Specific protein 6 (Gas6), which bridges the virus to the cells. Gas6 expression was also detected in human astrocytes. However, exposure to ZIKV had no effect on its expression (Figure 14). 


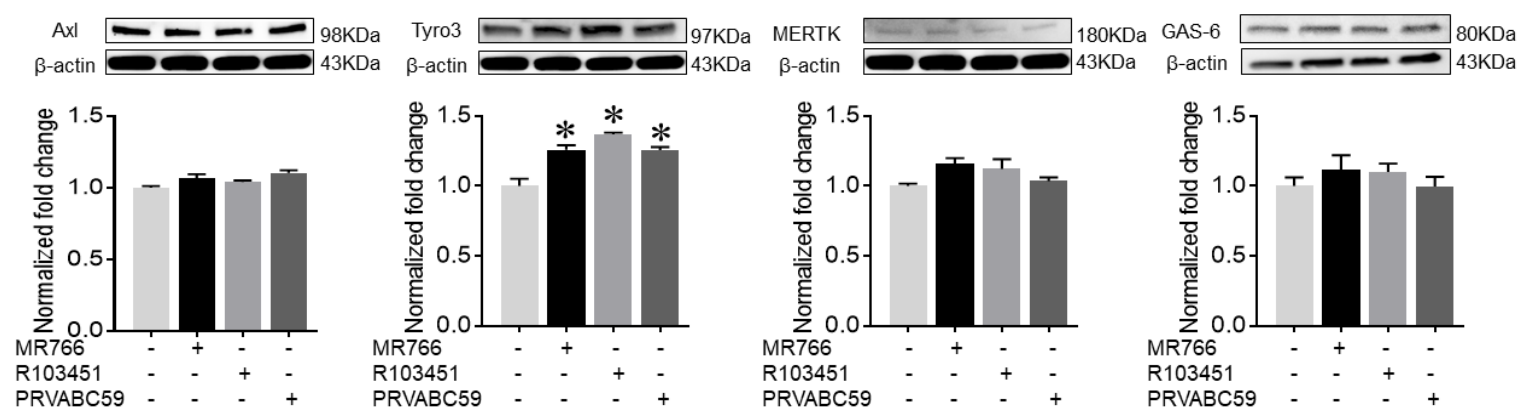

Figure 14. TAM receptors and their ligand Gas6 are expressed in human astrocytes. Protein expression of Axl, Tyro3, MERTK, and Gas6 was measured by western blot from the lysate of human astrocytes uninfected or infected with different strains of ZIKV at MOI of 0.1 for 24 hours. Data are presented as mean \pm SEM from 3 independent experiments and analyzed using one-way ANOVA with Bonferroni post hoc correction for multiple comparisons. *p $<0.05$ Vs Control.

\subsubsection{Inhibition of Axl suppresses ZIKV infection in human astrocytes}

To examine the role of Axl receptor in ZIKV entry, we pre-treated human astrocytes with a pharmacological inhibitor of Axl (R428) followed by infection with ZIKV. After $48 \mathrm{hpi}$, ZIKV infectivity of the astrocytes was determined by immunolabeling of ZIKV Env protein. The inhibition of host Axl receptor with R428 correlated dosedependently with a decrease in ZIKV infection (Figure 15A and B). Interestingly, we observed a decrease in ZIKV-induced secretion of IFN- $\beta$ by human astrocytes treated with R428 (Figure 15C), which correlates with a decrease in ZIKV infection. The decrease in IFN- $\beta$ secretion might be because of the viral suppression caused by Axl inhibition or the direct effect of Axl inhibition on downstream signaling molecules involved in the induction of IFN secretion. We further measured the effect of Axl inhibition in the number of virions released in the supernatants from the infected astrocytes as an indicator of viral replication. The viral titer in the supernatants was decreased with R428 mediated inhibition of Axl in ZIKV infected astrocytes irrespective of the viral strains. However, the inhibitory 
concentration of R428 may vary based on the strains. In fact, the Honduras strain (R103451) of ZIKV responded only to the highest concentrations of inhibitor $(1 \mu \mathrm{M})$ (Figure 15D). In order to determine any toxicity of R428, we examined the cell viability of astrocytes treated with increasing concentration of the compound. The toxicity was not detected up to the concentration of $5 \mu \mathrm{M}$ (Figure 16A).

Next, we confirmed the effect of Axl inhibition on ZIKV infection by genetically silencing Axl by siRNA in primary human astrocytes. We found about $60 \%$ decrease in axl expression and the transfection related toxicity was similar in siNT (Control) and siAxl transfected cells (Figure 16A and B). We found a similar decrease in viral infection by siRNA mediated silencing of Axl in the astrocytes (Figure 16C). Overall, the data suggest strain-dependent differences in the extent of inhibition of ZIKV entry by TAM inhibitors specifically Axl. Of note, some levels of infection or viral entry with all three strains were detected even after the treatment with a high dose of R428, suggesting an involvement of an alternative or secondary mechanism of ZIKV entry into astrocytes. Nevertheless, the data indicate that the Axl receptor plays an important role in ZIKV infection. 

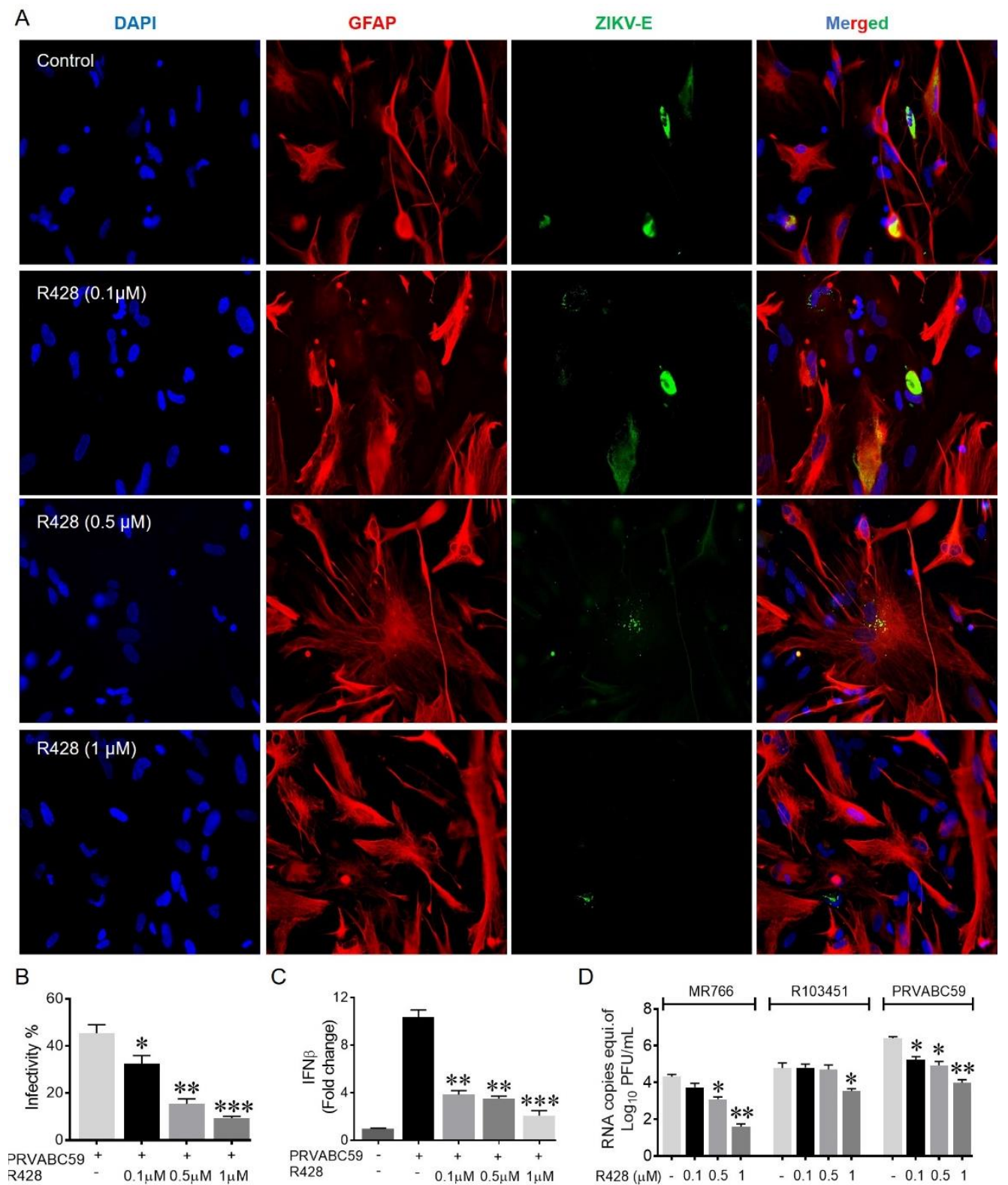

Figure 15. Inhibition of Axl by small molecule inhibitor (R428) decreases ZIKV infection in human astrocytes.

(A and B) ZIKV infectivity was measured by immunofluorescent staining of ZIKV Env protein in human astrocytes treated with increasing concentrations of Axl kinase inhibitor (R428). The cells were treated with R428 30 minutes prior to infection with ZIKV and fixed for immunostaining after 48 hours. (C) Secretion of IFN- $\beta$ was measured by ELISA from the supernatants collected from the infected astrocytes exposed to different concentrations of R428. (D) The infectivity was further confirmed by measuring viral titers in cell supernatants by RT-PCR. Data are presented as mean \pm SEM from 3 independent experiments and analyzed using one way or repeated measures ANOVA with Bonferroni post hoc correction. ${ }^{*} \mathrm{p}<0.05$ Vs Control, ${ }^{* *} \mathrm{p}<0.005$ Vs Control, and $* * * \mathrm{p}<0.001$ Vs Control. 

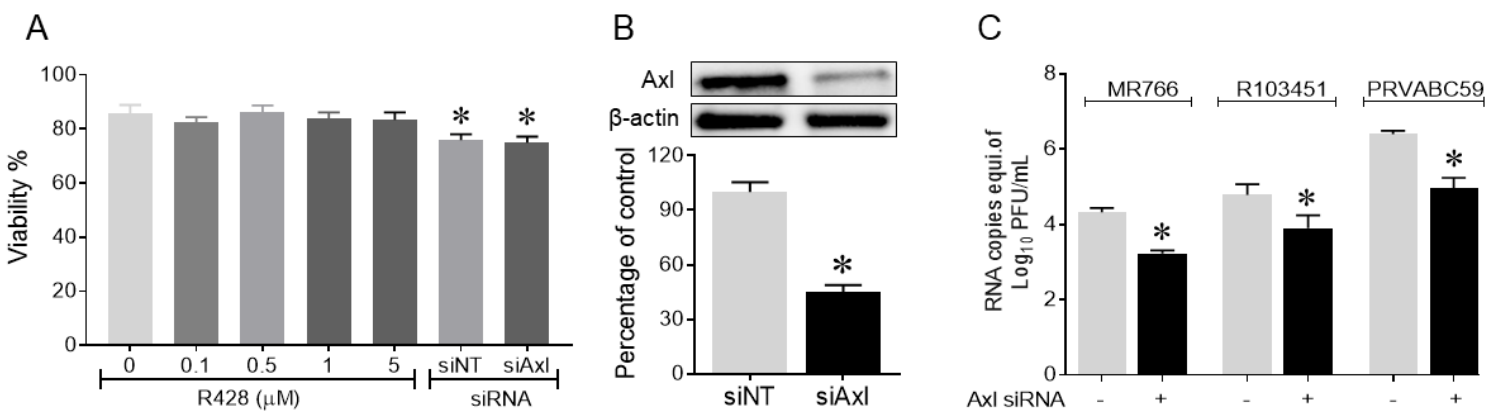

Figure 16. Genetic silencing of Axl also decreases ZIKV infection in human astrocytes.

Axl was knocked down by siRNA against Axl using lipofectamine 2000 transfection reagent. (A) Validation of Axl knockdown by immunoblotting. (B) The cells (both knocked down and parental) were infected with ZIKV at MOI of 0.1, and viral RNA titer was determined in the supernatants collected at $48 \mathrm{hpi}$. Data are presented as mean \pm SEM from 3 independent experiments and analyzed using unpaired t-test and two-way ANOVA with Bonferroni post hoc correction. *p $<0.05$ Vs Control.

\subsubsection{Inhibition of Axl enhances ZIKV-mediated inflammatory molecules secretion in human astrocytes}

We detected a concurrent decrease in IFN- $\beta$ response with a decrease in ZIKV infection in the astrocytes treated with R428 suggesting that the host immune response correlates with the viral burden in the cells. To further explore the impact of Axl inhibition on host inflammatory response, we measured IP-10 secretion by the infected cells exposed to R428 or having Axl knockdown. The data showed that there was no significant effect of Axl inhibition on the secretion of IP-10 by the astrocytes infected with MR766 and R103451 strains of ZIKV. However, pharmacological and genetic inhibition of Axl in astrocytes enhanced PRVABC59-induced secretion of IP-10 (Figure 17A and B). Since Axl is known to control the inflammatory response in the infected cells, inhibition of Axl may lead to exaggerated secretion of inflammatory molecules even at low viral titers as shown by the current data. 

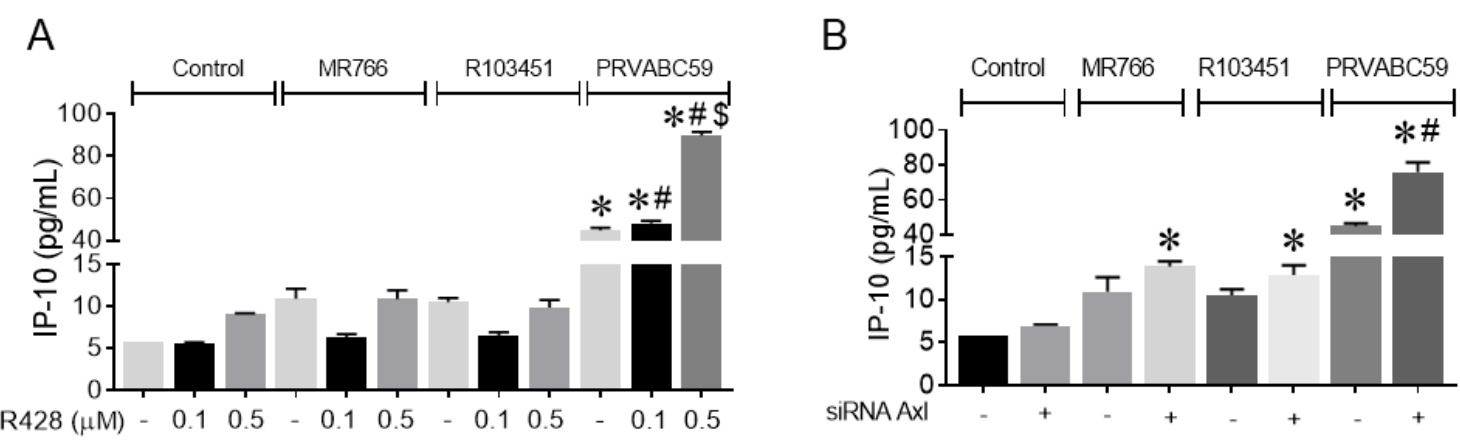

Figure 17. Axl antagonizes IFN response in human astrocytes.

(A) Secretion of IP-10, as a marker of IFN response, was measured by the ELISA method from the supernatants collected from the infected astrocytes exposed to different concentrations of R428. (B) Secretion of IP-10 was measured from the supernatants collected from the infected astrocytes with or without siRNA mediated Axl knockdown. Data are presented as mean \pm SEM from 3 independent experiments and analyzed using one way ANOVA with Bonferroni post hoc correction. *p $<0.05$ Vs Control.

\subsubsection{Inhibition of Tyro3 had no effect on ZIKV infection in human astrocytes}

Since we observed significant upregulation of Tyro3 receptor expression by ZIKV, we next inhibited Tyro3 by different concentration of c-Met specific small molecule inhibitor (BMS777607) in human astrocytes. Inhibition of Tyro3 had no effect on ZIKV infection in human astrocytes indicating that ZIKV cellular entry may be independent of Tyro3 (Figure 18A). Similarly, inhibition of tyro3 had no effect on ZIKV-mediated secretion of an inflammatory molecule, IP-10 (Figure 18B).

A

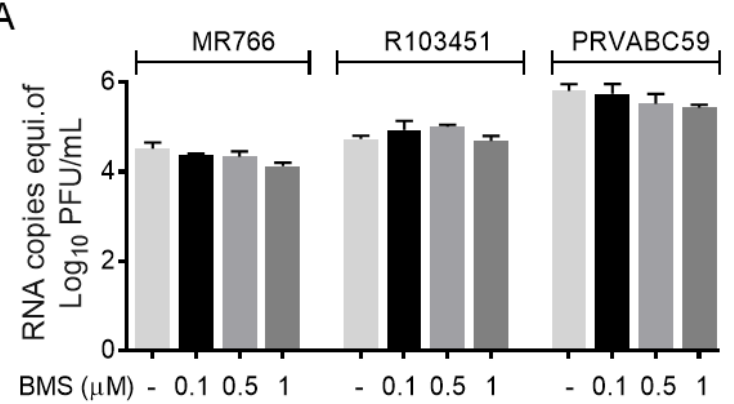

B

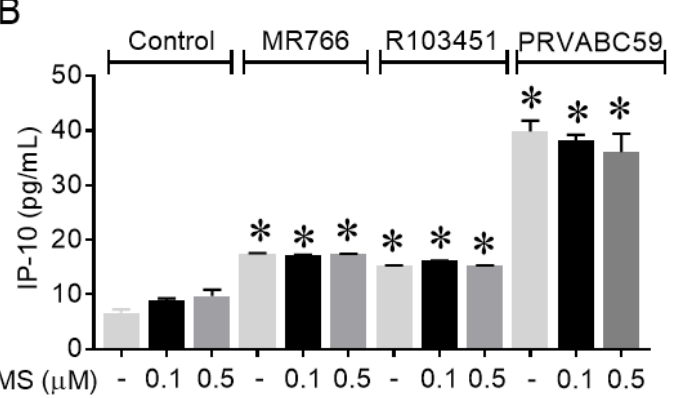

Figure 18. Inhibition of Tyro3 by small molecule inhibitor (BMS77607) has no effect on ZIKV infection in human astrocytes. 
(A) ZIKV RNA titer was measured by RT-PCR in the supernatants collected from human astrocytes treated with increasing concentrations of Tyro3 inhibitor (BMS777607). (B) IP10 was measured by ELISA from the supernatants collected from the infected astrocytes exposed or unexposed to different concentrations of BMS777607. Data are presented as mean \pm SEM from 3 independent experiments and analyzed using one way ANOVA with Bonferroni post hoc correction. *p $<0.05$ Vs Control.

\subsubsection{Inhibition of Tyro3 had no additive effect on ZIKV infection in human astrocytes treated with Axl inhibitor}

ZIKV infection in human astrocytes was significantly suppressed by Axl inhibitor, but not by Tyro3 inhibitors while using them separately. Next, we asked whether the combination of both Axl and Tyro3 inhibitors show a synergistic effect on ZIKV infection in human astrocytes. To this end, we treated human astrocytes with R428 and BMS777607 before infection with different strains of ZIKV. We chose the concentration of $1 \mu \mathrm{M}$ for both of the inhibitors based on previous pieces of literature [2, 12]. ZIKV RNA copies were measured in the supernatants collected from the infected astrocytes at 48 hpi. R428 alone and in combination with BMS777607 reduced the viral titers by about $2 \log _{10}$ copies. However, the Tyro3 inhibitor (BMS777607) had no additive effect on ZIKV titers with any of the viral strains (Figure 19A). We also measured the secretion of IP-10 by infected astrocytes exposed to either or both of the inhibitors. There were no significant changes in secretion of IP-10 with any of the inhibitors and with any of the viral strains (Figure 19B).

Overall, the data indicate that Tyro3 receptor is neither involved in ZIKV infection and associated inflammatory molecules secretion nor backs up the Axl receptor in doing so. 
A

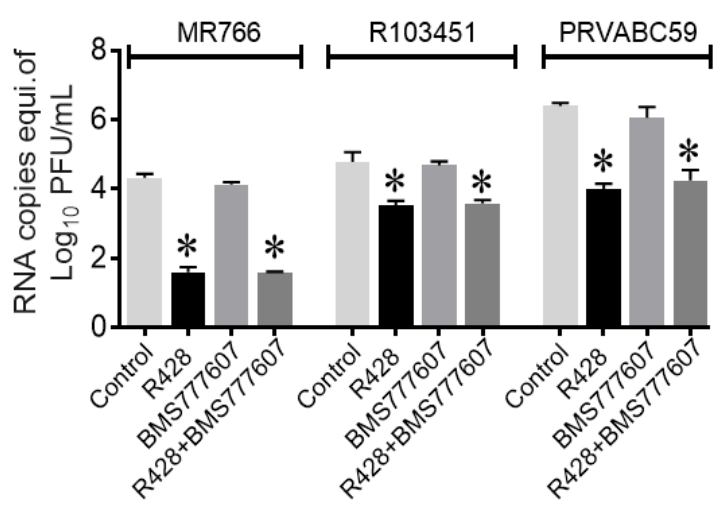

B

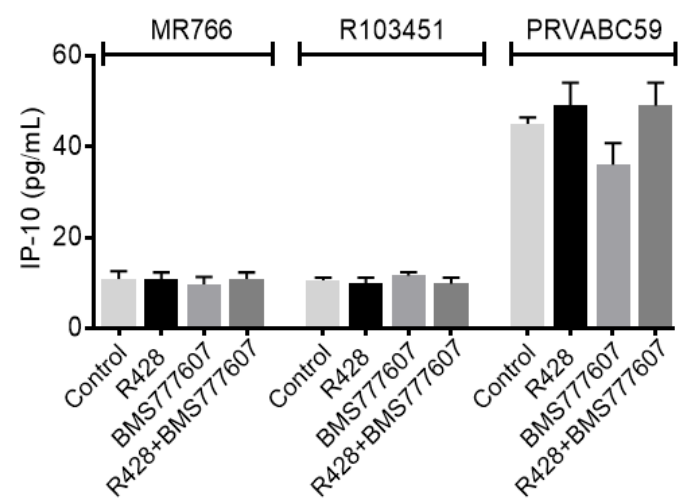

Figure 19. Inhibition of Tyro3 had no additive effect on ZIKV infection in human astrocytes treated with Axl inhibitor.

(A) ZIKV RNA titer was measured by RT-PCR in the supernatants collected from human astrocytes treated with both Axl and Tyro3 inhibitors at a concentration of $1 \mu \mathrm{M}$ each. (B) IP-10 was measured by ELISA from the supernatants collected from the infected astrocytes exposed or unexposed to Axl and Tyro3 inhibitors. Data are presented as mean \pm SEM from 3 independent experiments and analyzed using one-way ANOVA with Bonferroni post hoc correction. $* \mathrm{p}<0.05 \mathrm{Vs}$ control.

\subsubsection{Axl is necessary for ZIKV entry as well as for replication in human astrocytes}

By now, it is clear that Axl receptor is involved in ZIKV infection. However, whether Axl mediates only the viral entry into the cells or also facilitates viral replication is still not clear. Previously, we used a single time point for the measurement of viral titers. Therefore, in order to explore whether inhibition of Axl suppresses viral replication, we infected human astrocytes with Axl knocked down or pretreated with Axl inhibitor and measured viral titers at different time points, i.e., 24, 48 and 72 hpi. Here, we found that ZIKV replication was also repressed by Axl inhibition since there was no significant increase in viral titers with time. However, higher viral titers were detected at later time points than the initial time point in the infected control cells (without Axl inhibition) indicating viral replication (Figure 20A). 
Gas6 is the major Axl ligand probably facilitating the binding of ZIKV envelope proteins with the receptor for internalization of the virus. We next examined the role of Gas6 in ZIKV infection and replication. Since fetal bovine serum (FBS) used in culture media naturally contains Gas6 [13], we infected human astrocytes with ZIKV (PRVABC59) in the absence of FBS to minimize the exposure to Gas6. ZIKV infection of the astrocytes was significantly decreased in the absence of FBS supporting the tripartite model of ZIKV entry into the cells (Figure 20B). However, ZIKV replication was not as drastically decreased with reduced Gas6 as with Axl inhibition. The data indicate that though Gas6 is necessary for ZIKV entry, it may not facilitate ZIKV replication.

A

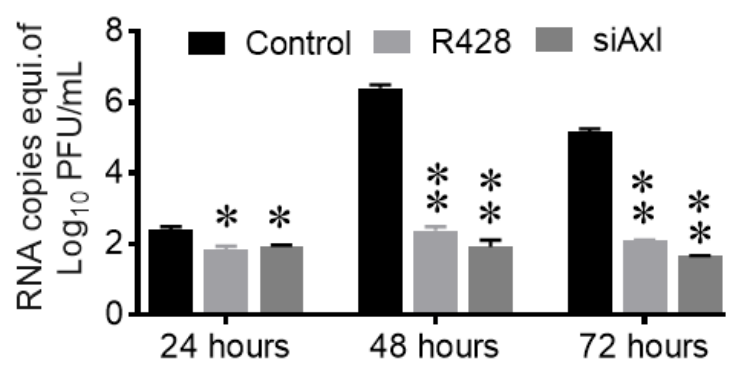

B

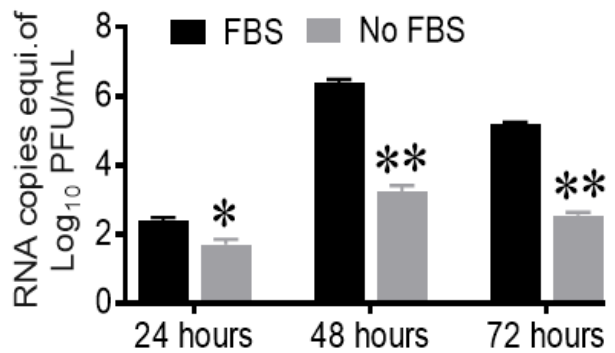

Figure 20. Axl facilitates ZIKV entry as well as replication within human astrocytes. (A) ZIKV RNA titer was measured by RT-PCR in the supernatants collected at indicated time points from infected human astrocytes untreated or treated with both Axl inhibitors at a concentration of $1 \mu \mathrm{M}$ each or Axl silenced using siRNA against Axl. (B) ZIKV RNA titer was measured by RT-PCR in the supernatants collected at indicated time points from infected human astrocytes under regular growth conditions or under serum-free conditions. Data are presented as mean \pm SEM from 3 independent experiments and analyzed using two way ANOVA with Bonferroni post hoc correction. * $p<0.05$ Vs Control, **p $<0.005$ Vs Control.

\subsection{Discussion}

Because of the devastating neurodevelopmental and neurodegenerative consequences of ZIKV infection, there is a dire need for a preventive and therapeutic 
solution. Currently, there are dozens of treatment approaches, and vaccine candidates under the evaluation with two of them progressed to human clinical trial phase 2 [14]. However, there is no specific treatment for the ZIKV infection yet. Most of the treatment approaches currently being studied are focused on directly targeting viral particles. The mutability of the viral genome, spatiotemporal heterogeneity of ZIKV transmission, the unpredictability of ZIKV epidemics and the wide ranges of clinical manifestations are some of the constraints for direct viral targeting approach. Therefore, we aim to identify potential host factor therapeutic target. We first examined the protein expression of putative ZIKV receptors, the TAM family receptors. Based on the expression pattern, we hypothesized that targeting Axl and/or Tyro3 could be a potential approach to suppress ZIKV infection.

Herein, we report that inhibition of Axl caused a decrease in ZIKV infection. Since several inhibitors of Axl receptors including R428 are currently in clinical trials against cancer that could be a unique antiviral treatment for ZIKV infection. The enveloped viruses such as DENV exploit Axl for viral entry by exposing phosphatidylserine residue on the external surface their envelope protein. By doing so, the virus mimics an apoptotic cell which Axl targets. The virus-mediated apoptotic mimicry allows virus not only to enter the permissive cells but also counteract type 1 IFN responses [15]. However, there is still a debate about whether Axl just facilitates ZIKV entry or also promote viral replication at the later point following viral entry. R428 neither blocks the viral entry or affect cell surface expression of Axl receptor, but significantly reduces viral titers. This supports the notion that Axl mediates innate immune response besides the direct involvement in the viral entry process. We further showed that targeting the Axl ligand Gas6 could be another approach to down-regulate the Axl function. In agreement with our findings MYD1 the Axl decoy 
receptor that sequesters Gas6, also reduces ZIKV infection. Gas6 is the ligand for TAM receptors and facilitates viral binding to Axl [2]. Previous studies have shown that Axl antagonizes type 1 IFN signaling and promotes ZIKV infection in the infected cells [3]. Detection of similar viral titers at different time point after ZIKV infection in the Axl inhibited astrocytes indicate that inhibition of Axl suppresses ZIKV entry as well as the replication thereafter. Though the exact mechanism how Axl facilities viral replication is still not clear, it has been shown that tyrosine kinase activity of the Axl is required for viral replication [16].

Of note, we couldn't observe the absolute suppression of the ZIKV infection even at high concentration of Axl inhibitor indicating that an alternative mechanism of cellular entry can't be ruled out. Besides viral entry to particular cell types, additional mechanisms including the ability of ZIKV to cross placental and BBB, virus-induced inflammation, the immune response of the patients may determine the extent of neuropathological manifestations related to ZIKV [14].

\section{References}

[1] Smit JM, Moesker B, Rodenhuis-Zybert I, Wilschut J. Flavivirus Cell Entry and Membrane Fusion. Viruses. 2011;3(2):160-71.

[2] Meertens L, Labeau A, Dejarnac O, Cipriani S, Sinigaglia L, Bonnet-Madin L, et al. Axl Mediates ZIKA Virus Entry in Human Glial Cells and Modulates Innate Immune Responses. Cell Reports. 2017;18(2):324-33.

[3] Chen J, Yang Y-f, Yang Y, Zou P, Chen J, He Y, et al. AXL promotes Zika virus infection in astrocytes by antagonizing type I interferon signalling. Nature Microbiology. 2018;3(3):302-9.

[4] Rankin EB, Giaccia AJ. The Receptor Tyrosine Kinase AXL in Cancer Progression. Cancers. 2016;8(11):103. 
[5] O'Bryan JP, Frye RA, Cogswell PC, Neubauer A, Kitch B, Prokop C, et al. axl, a transforming gene isolated from primary human myeloid leukemia cells, encodes a novel receptor tyrosine kinase. Molecular and Cellular Biology. 1991;11(10):5016-31.

[6] Camenisch TD, Koller BH, Earp HS, Matsushima GK. A novel receptor tyrosine kinase, Mer, inhibits TNF-alpha production and lipopolysaccharide-induced endotoxic shock. Journal of immunology (Baltimore, Md : 1950). 1999;162(6):3498-503.

[7] Jung CY, Kim SY, Lee C. Carvacrol Targets AXL to Inhibit Cell Proliferation and Migration in Non-small Cell Lung Cancer Cells. Anticancer research. 2018;38(1):279-86.

[8] Hamel R, Dejarnac O, Wichit S, Ekchariyawat P, Neyret A, Luplertlop N, et al. Biology of Zika Virus Infection in Human Skin Cells. journal of virology. 2015;89(17):8880-96.

[9] Hastings AK, Yockey LJ, Jagger BW, Hwang J, Uraki R, Gaitsch HF, et al. TAM receptors are not required for Zika virus infection in mice. Cell Rep. 2017;19(3):558-68.

[10] Li F, Wang PR, Qu LB, Yi CH, Zhang FC, Tang XP, et al. AXL is not essential for Zika virus infection in the mouse brain. Emerg Microbes Infect. 62017. p. e16-.

[11] Cui L, Zou P, Chen E, Yao H, Zheng H, Wang Q, et al. Visual and Motor Deficits in Grown-up Mice with Congenital Zika Virus Infection. EBioMedicine. 2017;20:193-201.

[12] Zeng J-Y, Sharma S, Zhou Y-Q, Yao H-P, Hu X, Zhang R, et al. Synergistic Activities of MET/RON Inhibitor BMS-777607 and mTOR Inhibitor AZD8055 to Polyploid Cells Derived from Pancreatic Cancer and Cancer Stem Cells. Molecular Cancer Therapeutics. 2014;13(1):37-48.

[13] Fernández-Fernández L, Bellido-Martín L, García de Frutos P. Growth arrestspecific gene 6 (GAS6). Thromb Haemost. 2008;100(10):604-10.

[14] Wilder-Smith A, Vannice K, Durbin A, Hombach J, Thomas SJ, Thevarjan I, et al. Zika vaccines and therapeutics: landscape analysis and challenges ahead. BMC medicine. 2018;16(1):84-.

[15] Paolino M, Penninger JM. The Role of TAM Family Receptors in Immune Cell Function: Implications for Cancer Therapy. Cancers. 2016;8(10):97. 
[16] Meertens L, Carnec X, Lecoin Manuel P, Ramdasi R, Guivel-Benhassine F, Lew E, et al. The TIM and TAM Families of Phosphatidylserine Receptors Mediate Dengue Virus Entry. Cell Host \& Microbe. 2012;12(4):544-57. 


\section{CHAPTER 4: TLR3 REGULATES ZIKV INFECTION AND ASSOCIATED HOST INFLAMMATORY RESPONSES IN HUMAN GLIAL CELLS}

\subsection{Introduction}

ZIKV neurotropism and associated neurological and congenital complications have already been established [1]. Despite the persistent effort of the scientific community, the mechanism regulating ZIKV virus replication and associated pathologies in the brain is yet to be understood. Being a flavivirus, ZIKV can be expected to behave similarly to other flaviviruses. Flaviviruses exploit the ER membranes by rearranging them to form a replication platform that facilitates the assembly of newly synthesized viral components and complete the virions packaging before trafficking to the Golgi complex [2-4]. Synthesis of excessive viral protein in the close vicinity of the ER may, in turn, lead to ER stress. Cellular UPR pathway, which is a homeostatic signaling pathway counteracting ER stress mitigates unfolded protein load by pro-survival mechanisms such as ER membrane expansion, decreased influx of proteins into ER, induction of transcription of key components of protein folding [5, 6]. If the stress is not under control, UPR induces ERphagy or apoptosis. UPR is induced via either of three arms or stress sensors; PERK, IRE1 $\alpha$ and ATF6 [5]. ZIKV induces UPR pathway in human fetal brain cortex, mouse embryonic brain and in vitro hNSCs, while PERK inhibitor rescues ZIKV associated neurogenic defects [7]. The current understanding of the ZIKV-interaction with ER stress and the related pathway is limited. Therefore, we aimed to investigate the role of the UPR pathway in ZIKV infection and associated neuropathologies.

ZIKV infection in the acute and convalescent phases induces secretion of various pro-inflammatory molecules including IP-10, RANTES and MCP-1, IL-6 [8]. We have 
already assessed the levels of inflammatory molecules induced by different strains of ZIKV in human astrocytes and microglia. However, the exact mechanism of ZIKV induced inflammatory molecules secretion is still not clear. Activation of the TLR pathway and subsequent upregulation of pro-inflammatory molecules secretion by glial cells is a key component of neuroinflammation associated with many neurological disorders [9]. TLR pathway mediates an early innate antiviral response by inducing the IFN signaling besides the induction of inflammatory molecules secretion $[10,11]$. Since ZIKV is reported to activate TLR3 in human organoid cells and murine neurospheres, leading to perturbation of genes related to neurodevelopment and reducing the organoid volume as seen in clinical microcephaly [12], we hypothesized that TLR3 pathway might be involved in ZIKV infection and associated neuropathologies in the brain.

The goal of the specific aim 2 was to determine the mechanisms of ZIKV mediated neuropathology specifically neuroinflammation using primary human astrocytes infected with spatiotemporally different strains of ZIKV. Our findings showed that ZIKV induces the UPR pathway via PERK and TLR pathway via TLR3 in human astrocytes. We further report that the TLR3 pathway is an important player in ZIKV replication and the release of inflammatory molecules in astrocytes, irrespective of viral strains.

\subsection{Materials and methods}

\subsubsection{Cell cultures, treatment, and ZIKV infection}

Human primary astrocytes were grown and maintained as explained in the previous chapters. The TLR3/dsRNA complex inhibitor (a thiophenecarboxamidopropionate compound) and TLR3 agonist (Poly I:C) were purchased from Millipore Sigma and Sigma Aldrich, respectively. To infect with each viral strain, viral stock was diluted in the 
appropriate media based on cell types. Cells were infected with the ZIKV as in the previous experiments.

\subsection{2. mRNA expression profiling}

Expression of genes involved in the UPR pathway and TLR pathway was determined by RT-PCR profilers array. Briefly, human astrocytes were infected with three strains of ZIKV (MR766, R103451, and PRVABC59) for 24 hours. RNA was isolated using the RNeasy Mini Kit (Qiagen, Valencia, CA, USA). Purity of the RNA (O.D. 260/280 nm absorbance ratio of at least 2.0) was measured by a microspot RNA reader (Synergy HT Multi-Mode Microplate Reader from BioTek) and 0.5 $\mu \mathrm{g}$ of RNA was used for cDNA synthesis using Qiagen's RT2 First Strand Kit (Cat. 330401, Qiagen, Valencia, CA, USA) as per the manufacturer's protocol after eliminating genomic DNA. Then, the synthesized cDNA was mixed with RT2 SYBR Green/ROX PCR master mix (Cat. 330520, Qiagen, Valencia, CA, USA) to run RT-PCR in 96 well plates according to manufacturer's protocol.

\subsubsection{Immunoblotting}

Immunoblotting was done as described in previous sections. Primary antibodies against TLR3 (Cat. sc-28999, 1:200), TLR4 (Cat sc-30002, 1:200), TLR5 (Cat. sc-10742, 1:200), MyD88 (Cat. sc-74532, 1:200), PERK (Cat. sc-377400, 1:200), IRE1 $\alpha$ (Cat. sc-

390960, 1:200), ATF6 $\alpha$ (Cat. sc166659, 1:200), DDIT3 (Cat. sc-7351, 1:200) and $\beta$ actin (Cat. sc-47778, 1:200) (Santa Cruz Biotechnology, Santa Cruz, CA, USA) were followed by incubation with a respective secondary antibody conjugated to horseradish peroxidase (Millipore, Billerica, MA, USA) used at specified dilution. 


\subsubsection{Immunocytochemistry}

Viral infectivity with each viral strain was measured by fluorescent immunolabeling. Briefly, cells were fixed in $4 \%$ paraformaldehyde, permeabilized with $0.1 \%$ Triton X-100, and blocked in $10 \%$ milk/0.1\% goat serum. Human astrocytes were immunolabeled with mouse anti-GFAP antibody (Cat. MAB360, Millipore, Boston, MA, USA) and rabbit anti-ZIKV-E antibody (Cat. GTX133314, Genetex, Irvine, CA, USA). Immunoreactivity was visualized with secondary antibodies from molecular probes (Carlsbad, CA, USA). 4',6-diamidino-2-phenylindole (DAPI) was used to label cell nuclei. The Images were analyzed using an inverted fluorescence microscope with a 560 Axiovision camera (Zeiss, Germany).

\subsubsection{NF-кB Nuclear localization assay}

Nuclear and cytoplasmic fractions were separated using fractionation buffer (HEPES-20mM, KCL-10mM, MgCl2-2mM, EDTA-1mM, DTT-1 mM, and protease and phosphatase inhibitor cocktail) and differential centrifugations. Both the nuclear and cytoplasmic NF- $\mathrm{KB}$ was measured by immunoblotting as described above. Immunofluorescent staining for NF- $\mathrm{KB}$ and GFAP was performed to locate and quantify

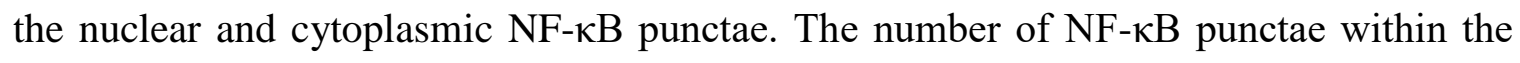
nuclei and outside the nuclei but within the cytoplasm were counted from at least 50 of the 40X microscopic fields selected randomly.

\subsubsection{Silencing of PERK and TLR3 in human astrocytes}

Human primary astrocytes were transfected with siRNA against PERK and TLR3 using Lipofectamine 2000 (Invitrogen, Carlsbad, CA, USA) or Fugene HD (Promega, Madison, WI, USA). siRNA and transfection reagent (ratio, 1:2) were pre-incubated for 20 
min in OptiMEM medium before addition to the seeded cells. Knockdown of targets was confirmed in cell lysates by western blot for the protein expression of PERK and TLR3.

\subsubsection{Statistical analysis}

Results are reported as mean \pm SEM of 3-6 independent experiments each with more than three samples. Data were analyzed using analysis of variance (ANOVA) followed by appropriate post hoc test for multiple comparisons (GraphPad Software, Inc., La Jolla, CA, USA). An alpha level (p-value) of $<0.05$ was considered significant.

\subsection{Results}

\subsubsection{ZIKV induced ER stress and unfolded protein response (UPR) pathway in human astrocytes}

Since flaviviruses including ZIKV replicate in close association with the ER and we reasoned that ZIKV replication mediates ER stress responses that could potentially be linked to high levels of inflammation and cell death [2], we, first, investigated ER stress and related pathways. We measured changes in mRNA expression of genes involved in the unfolded protein response pathway using gene expression profile arrays from RNA isolated after infection with the different strains of ZIKV in human astrocytes. Infection with the Asian strains (R103451 and PRVABC59) showed increased expression of the DNA damage-inducible transcript (DDIT3) and peptidyl-prolyl cis-trans isomerase A (PPIA) genes, while infection with the African strain caused increased expression of fork-head box 06 (FOX06), mitogen-activated protein kinase-10 (MAPK10), Insulin inducible gene 1 (INSIG1) and PPIA genes (Figure 21A). DDIT3 is a member of the CCAAT/enhancerbinding protein $(\mathrm{C} / \mathrm{EBP})$ family of a transcription factor which is activated by ER stress to promote apoptosis. PPIA binds to unfolded proteins including viral proteins; the FOX06 
gene is involved in DNA damage and cell cycle regulation, the MAPK10 (also called Janus kinase-3 or JNK3) gene, regulates multiple biological processes including stress-induced neuronal apoptosis while INSIG1 regulates lipid metabolism [13-16].

The upregulated genes are mainly related to DNA damage and cell cycle regulation. Therefore, we further measured the protein expression of representative genes involved in DNA damage induction (DDIT3) and cell cycle regulation (MAPK10). Infection with the Honduras strain (R103451) shows induction of protein expression of DDIT3 (Figure 21B). We observed upregulation of the MAPK10 gene in cells infected with the African strain (MR766), and given that MAPK signaling pathway is known to induce inflammation, we examined the expression level of JNK, a major component of MAPK signaling, by western blot. Infection with MR766 caused moderate induction in the expression levels of the JNK protein (Figure 21C), which also acts as a downstream effector molecule and modulator for the UPR pathway [17].
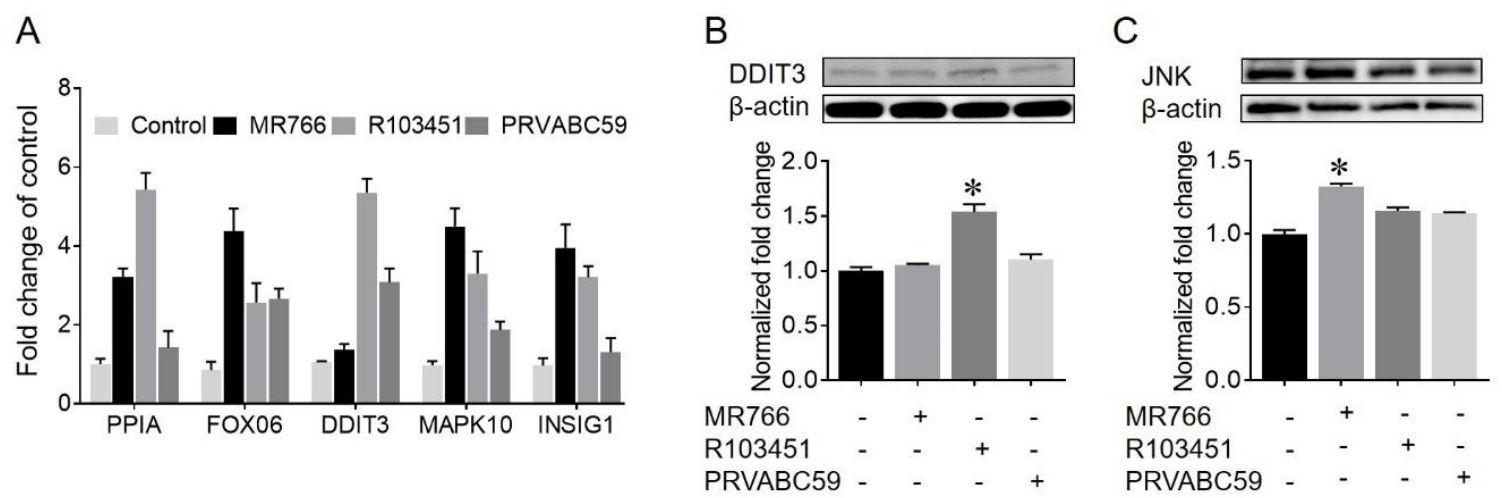

Figure 21. ZIKV induces DNA damage and ER stress in human astrocytes. Human astrocytes were infected with ZIKV (MR766, R103451, and PRVABC59) at an MOI of 0.1. After $48 \mathrm{hpi}$, RNA was extracted from cell lysates, and RT-PCR arrays were performed to determine RNA expression of genes involved in human unfolded protein response pathway. (A) Genes significantly altered by ZIKV infection are presented in the graph. (B and C) Immunoblotting for protein expression of DDIT3 and JNK from cell lysates from uninfected and ZIKV infected human astrocytes. Data are presented as mean 
\pm SEM from 3 independent experiments and analyzed using one-way ANOVA with Bonferroni post hoc correction. *p <0.05 Vs Control.

We further analyzed the activation and phosphorylation of the proteins involved in the ER-stress and UPR pathways by western blot (Figure 22A-F). The Uganda strain (MR766) caused upregulation of the protein kinase R (PKR)-like endoplasmic reticulum kinase (PERK), inositol-requiring enzyme $1 \alpha$ (IRE-1 $\alpha)$ and activating transcription factor$6 \alpha(\mathrm{ATF} 6 \alpha)$ proteins, while infection with the Honduran strain induced expression of IRE$1 \alpha$ and ATF6 $\alpha$. The Puerto-Rican strain induced PERK and IRE-1 $\alpha$. Increased phosphorylated PERK (p-PERK) was detected with the Asian strain of ZIKV; however, phospho-IRE-1 $\alpha$ was not increased with any of the viral strains. The cleaved form of ATF6 $\alpha$ was slightly increased with Uganda (MR766) and Honduras (R103451) strains (Figure 22A-F). Overall, the data showed that ZIKV induces the UPR pathway by probably by activation and phosphorylation of PERK. 
A
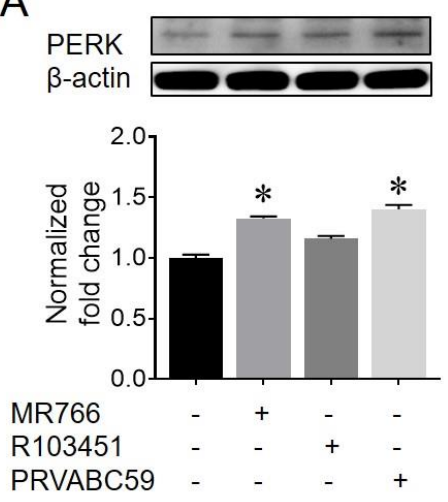

$\mathrm{D}$
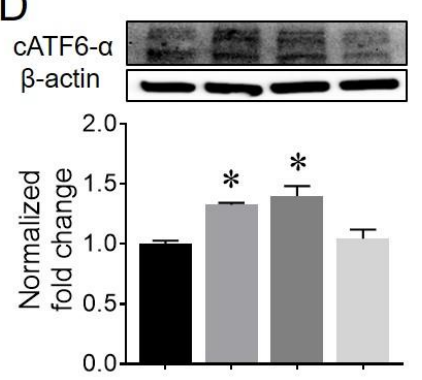

MR766

R103451

PRVABC59 -
B
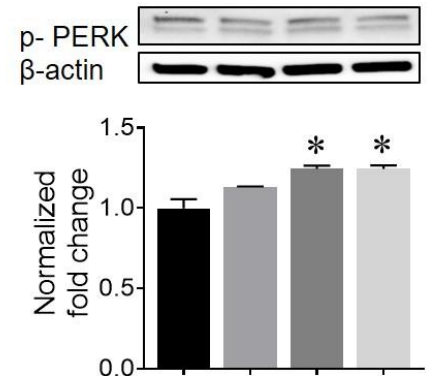

MR766

R103451

PRVABC59

E
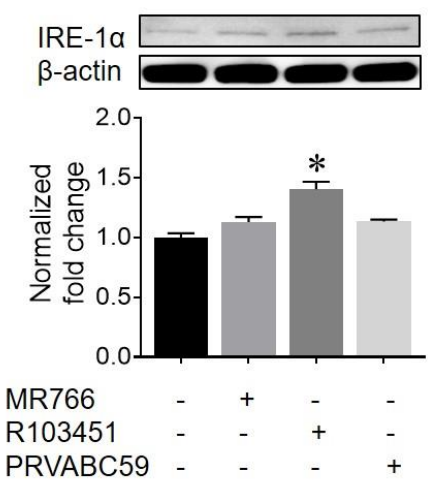

C
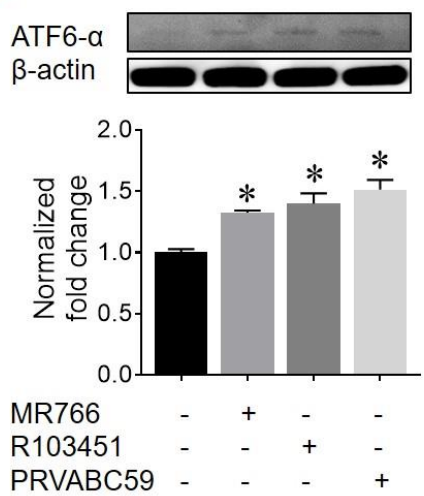

$\mathrm{F}$
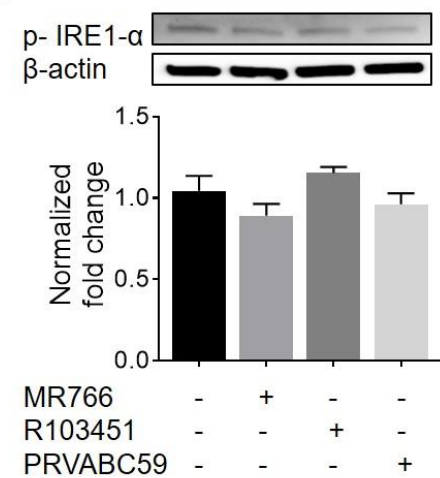

Figure 22. ZIKV induces unfolded protein response in human astrocytes through PERK.

Immunoblotting for the detection of protein expression and phosphorylation of PERK, expression, and phosphorylation of IRE- $1 \alpha$, and activation and cleavage of ATF- $6 \alpha$ in human astrocytes after $48 \mathrm{hpi}$ with ZIKV at MOI of 0.1 . Data are presented as mean \pm SEM from 3 independent experiments and analyzed using one-way ANOVA with Bonferroni post hoc correction. $* \mathrm{p}<0.05 \mathrm{Vs}$ Control.

\subsubsection{Inhibition of UPR pathway suppresses ZIKV replication in human astrocytes}

In order to investigate the role of UPR pathway in ZIKV infection and associated immune inflammatory response, we downregulated UPR using siRNA against PERK, the protein activated by ZIKV in our current study and has previously been reported to influence ZIKV mediated pathology in neurons [7]. The siRNA mediated downregulation of PERK resulted in a significant decrease in ZIKV replication in human astrocytes (Figure 23A). However, the decrease was less than one $\log _{10}$. Silencing of PERK caused a slight 
increase in IP-10 secretion in ZIKV (PRVABC59) infected human astrocytes (Figure 23B). Since the effect of PERK silencing was minimal on ZIKV infection, and the secretion of IP-10 did not correlate with viral titers, we explored for other cellular pathway involved in ZIKV infection.

A

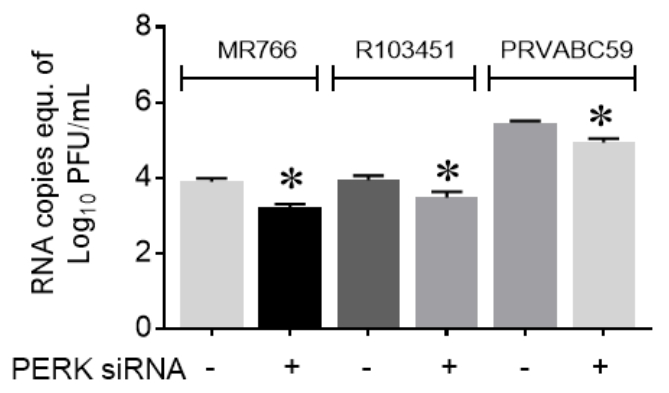

B

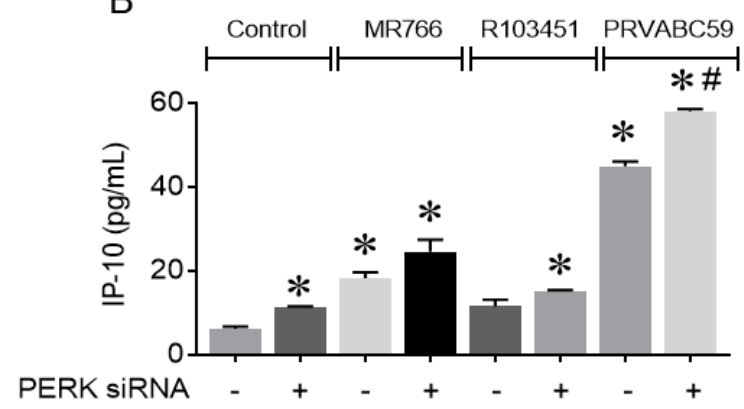

Figure 23. Silencing of PERK slightly reduces ZIKV replication in human astrocytes.

TLR3 in the human astrocytes was knocked down by siRNA against TLR3 using lipofectamine 2000 transfection reagent. The cells (both knocked down and parental astrocytes) were infected with ZIKV at MOI of 0.1. (A) RT-PCR using ZIKV specific primers was performed to determine viral titers from the RNA extracted from the supernatants. (B) Secretion of IP-10 was measured by ELISA from the supernatants collected at 48 hpi. Data are presented as mean \pm SEM from 3 independent experiments and analyzed using one way ANOVA with Bonferroni post hoc correction. *p $<0.05$ Vs Control.

\subsubsection{ZIKV induce TLR3 signaling pathway in human astrocytes}

Toll-like receptors (TLRs) are essential signaling pathways involved in the innate immune defense mechanism since they recognize and respond to pathogens. Despite the fact, little is known about TLR-mediated ZIKV replication and pathology in glial cells. Modulation of the TLR pathway by ZIKV-infection in primary human astrocytes was examined by gene expression profiling (Figure 24A), and protein expression was confirmed by western blot (Figure 22B-F). mRNA expression profile showed upregulation 
in the levels of TLR3 with Asian strains, upregulation in TLR4 with all three strains and down-regulation of TLR5 with all three strains of ZIKV. Also, the ETS transcription factor1 (ELK1), which is activated by MAPK signaling and involved in multiple functions ranging from neuronal differentiation to chromatin remodeling [18], was increased with MR766, however, was decreased by PRVABC59 (Figure 24A). Similarly, mRNA expressions of genes involved in TLR signaling pathways such as TICAM2, IRF3, ECSIT, EIF2K2, and HSPA1A were induced by the Uganda strain, whereas IRF3, ECSIT, FOS, and IFN- $\beta$ were upregulated by the Puerto-Rican strain. On the other hand, Pellino-1, a regulator of TLR signaling pathway [19], was downregulated with the Uganda strain (MR766) (Figure 24A).
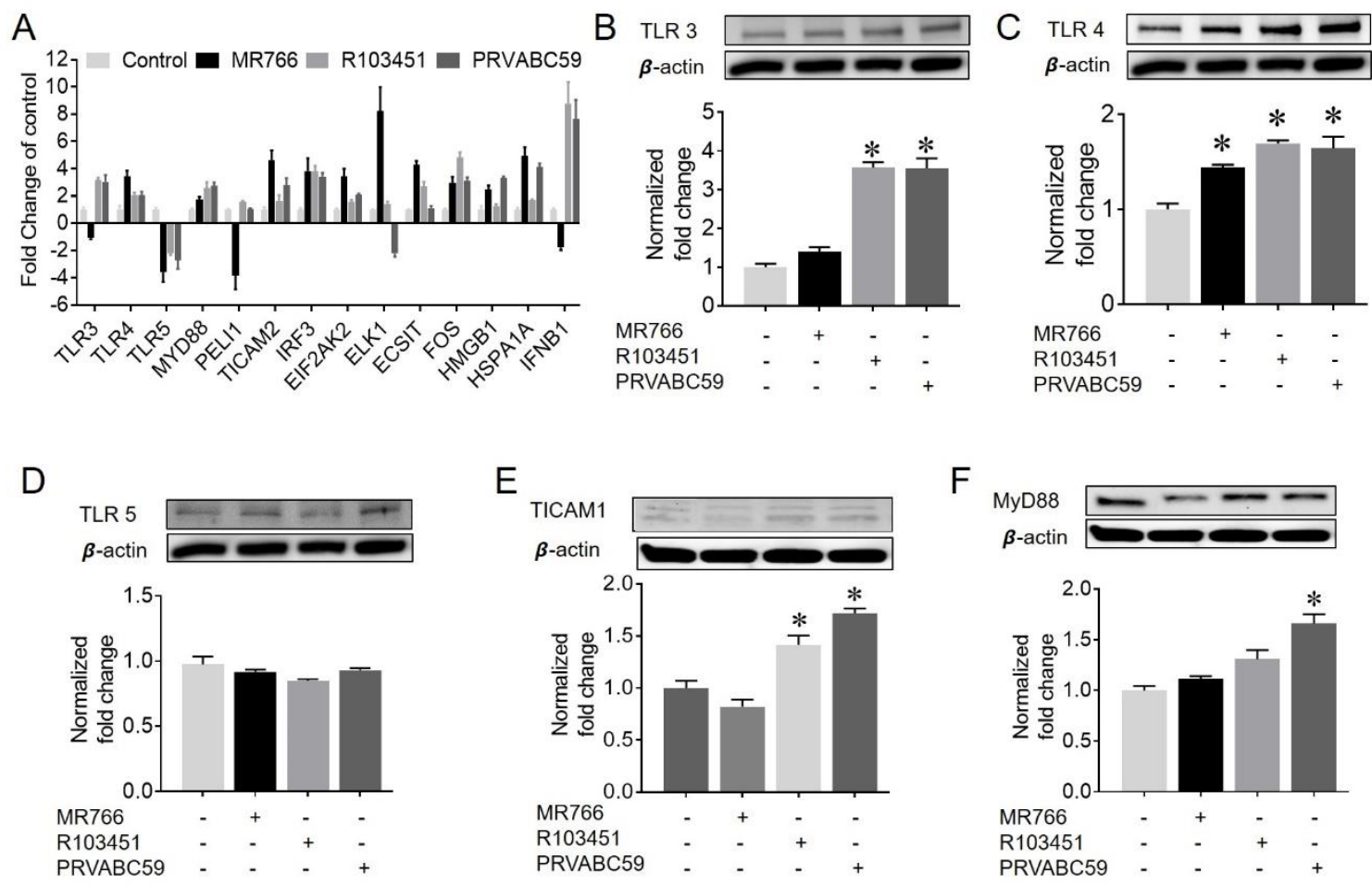

Figure 24. ZIKV perturbs TLR signaling pathway in human astrocytes.

(A) Human astrocytes were infected with different (indicated) strains of ZIKV at MOI of 0.1 , and cellular RNA was extracted after 48 hpi for determining the expression of genes involved in TLR signaling pathway by RT-PCR. A 2-fold change in gene expression was 
used as a cut off value. Immunoblotting for the detection of protein expression of TLRs (TLR-3, 4 and 5) and the TLR adaptor proteins TICAM1 and MyD88 in human astrocytes after 48 hpi with ZIKV at MOI of 0.1. Data are presented as mean \pm SEM from 3 independent experiments and analyzed using one way ANOVA with Bonferroni post hoc correction. *p <0.05 Vs Control.

Together with mRNA expression, TLR3 protein expression levels were also upregulated by around 3-fold in astrocytes infected with either R103451 or PRVABC59

(Figure 24B), TLR4 protein expression was upregulated by approximately 2-folds with all three strains (Figure 24C), while minor changes were detected in TLR5 expression level (Figure 24D). An adaptor protein involved in the TLR signaling pathways, TICAM1 and MyD88, were significantly increased in astrocytes infected with the Puerto Rican strains (PRVABC59) (Figure 24E and F). TICAM1, which is the adaptor protein involved in the TLR3 signaling pathway, was also induced by the other Asian strain (R103451).

\subsubsection{ZIKV induces activation of IRF3 and nuclear localization of NF- $\mathrm{KB}$}

Since the activation of TLR3 and recruitment of adaptor proteins leads to downstream signaling pathway involving the activation of two important transcription factor, IRF3, and $\mathrm{NF}-\kappa \mathrm{B}$, we next measured the effect of ZIKV on the expression of these factors. We detected increased expression of IRF3 in ZIKV infected astrocytes indicating that activated TLR3 pathway induces IFN responses via IRF3 induction (Figure 25A). NF- $\kappa$ B is a regulator of the immediate early host-pathogen inflammatory and cell survival responses $[20,21]$. We detected a significant upregulation in protein expression of $\mathrm{NF}-\kappa \mathrm{B}$ in human astrocytes infected with the Puerto-Rican strain (PRVABC59) (Figure 25B). Activation of $\mathrm{NF}-\kappa \mathrm{B}$ is followed by degradation of associated inhibitory molecule I $\kappa \mathrm{B} \alpha$ and nucleation localization of NF- $\mathrm{NB}$ for transcriptional regulation of various genes. Therefore, we 
examined both the cytoplasmic and nuclear fraction of the NF- $\kappa \mathrm{B}$ by western blot and immunofluorescence labeling of NF-kB (p65) of the infected astrocytes. We observed

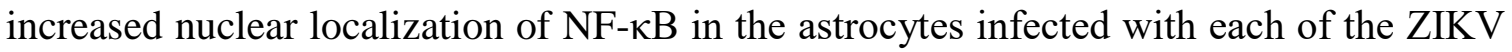
strains (Figure 25C-E).
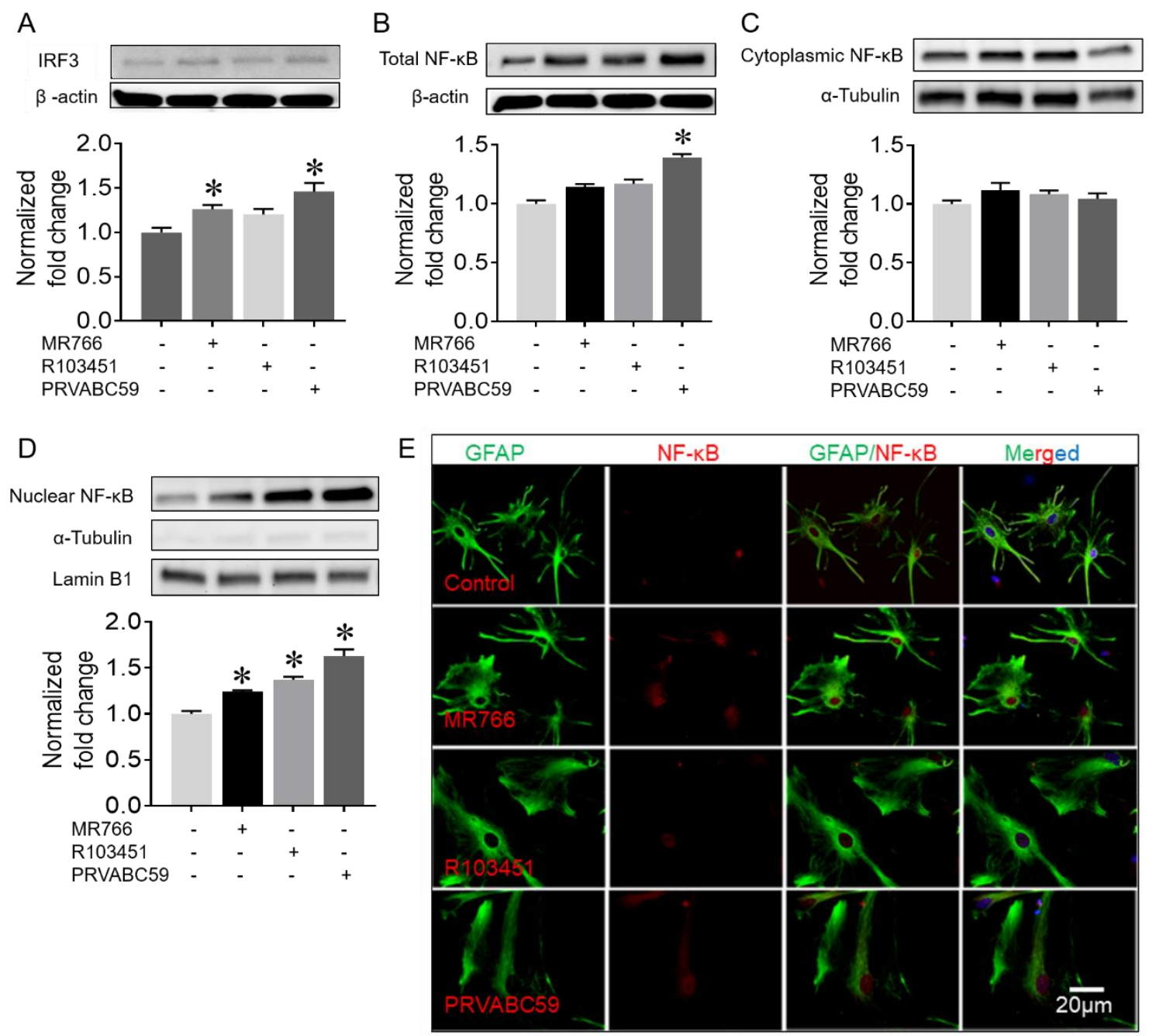

Figure 25. ZIKV induces downstream signaling molecules in the TLR3 signaling pathway; IRF3 and NF-кB.

(A and B) Immunoblotting for the detection of protein expression of TLR3 downstream signaling molecules (IRF3 and NF- $\kappa \mathrm{B}$ ) in human astrocytes after 48 hpi with ZIKV at MOI of 0.1. (C and D) Cytoplasmic and nuclear fractions of NF- $\kappa \mathrm{B}$. (E) Immunofluorescent staining of human primary astrocytes with antibody for GFAP, and $\mathrm{NF}-\kappa \mathrm{B}$, to determine nuclear and cytoplasmic localization of NF- $\kappa$ B. DAPI was used for labeling nuclei. Data are presented as mean \pm SEM from 3 independent experiments and analyzed using oneway ANOVA with Bonferroni post hoc correction. *p $<0.05$ Vs Control. 


\subsubsection{TLR3 regulates ZIKV replication in human astrocytes}

Since TLR3 is the highest up-regulated protein in our experiments and is reported to be involved in viral infection and associated pathogenesis [22], we investigated whether this receptor protein is involved in ZIKV replication and associated inflammatory responses in astrocytes. We induced TLR3 signaling by using TLR3 agonist poly I: C before and during infection with ZIKV. TLR3 signaling was downregulated by genetic silencing with siRNA against the TLR3 (Figure 26A) or with pharmacological inhibitor (a thiophenecarboxamidopropionate compound), which inhibits TLR3/dsRNA complex formation. Following the respective treatment, astrocytes were infected with three different strains of ZIKV as mentioned earlier. Though statistically insignificant, induction of TLR3 with agonist slightly increased the replication of ZIKV. Whereas, genetic and pharmacological inhibition of TLR3 signaling showed a significant decrease in viral titers in the supernatant collected from the cells infected with each of the three strains with the African (MR766) strain showing the greatest decrease in viral titer after silencing with siRNA (Figure 26B). 
A
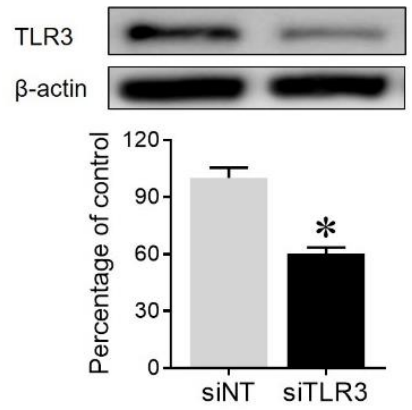

C
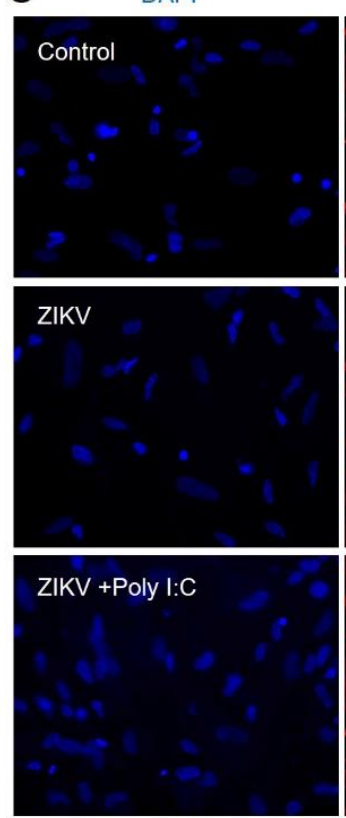

ZIKV +TLR3I

B

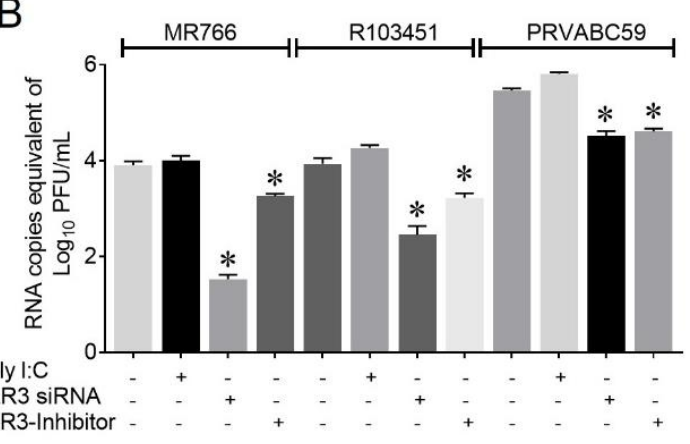

GFAP
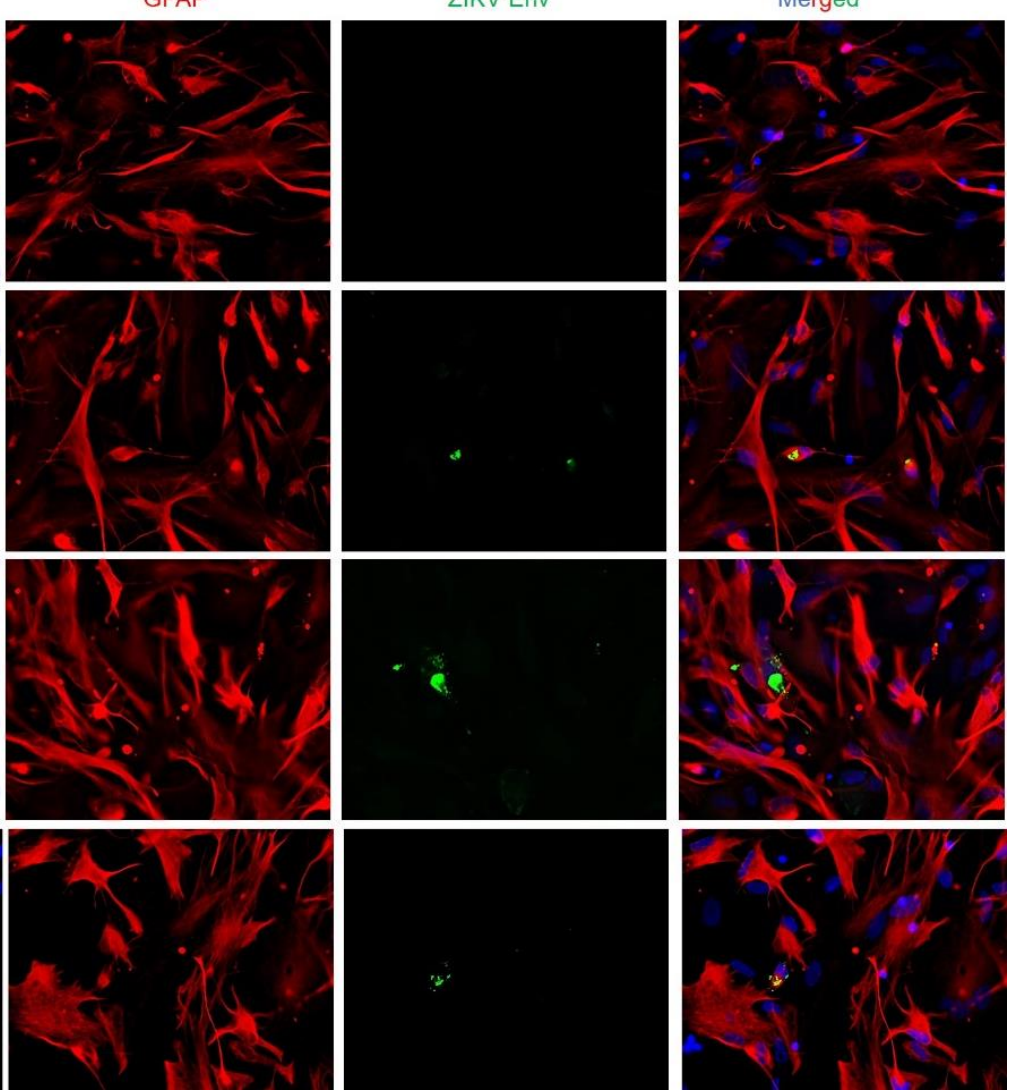

Figure 26. Silencing of TLR3 reduces ZIKV infection and replication.

Human astrocytes were infected with three different strains of ZIKV (MR766, R103451, and PRVABC59) at an MOI of 0.1. Sets of the experiment included astrocytes treated with $5 \mu \mathrm{g} / \mathrm{mL}$ of Poly I: C (TLR3 agonist), or with TLR3 silenced with siRNA (A) or inhibited with a thiophenecarboxamidopropionate compound (TLR3/dsRNA complex inhibitor). (B) RNA was extracted from the supernatant and RT-PCR using ZIKV specific primers was performed to determine viral titers. (C) ZIKV infectivity was measured by immunofluorescent staining of ZIKV Env protein and an astrocyte marker GFAP after 24 hpi with PRVABC59 strain. Data are presented as mean \pm SEM from 3 independent experiments and analyzed using one-way ANOVA with Bonferroni post hoc correction. *p $<0.05$ Vs Control. 
Similarly, we measured the ZIKV (PRVABC59) infectivity in the presence of TLR3 agonist or antagonist. We observed a similar trend with a slight increase in infectivity with TLR3 agonist and a decrease in infectivity with TLR3 antagonist. However, the changes in infectivity were not significant (Figure 26C). The data indicate that TLR3 is involved in ZIKV replication but not necessarily in viral entry or permissiveness in the astrocytes.

\subsubsection{TLR3 regulates ZIKV associated host immune inflammatory responses in human astrocytes}

Next, we asked whether TLR3 also mediates ZIKV-induced inflammatory molecules secretion in human astrocytes. Similar to the previous experiment, we induced TLR3 signaling by using poly I: C and downregulated TLR3 by siRNA against the TLR3 or with the pharmacological inhibitor in human astrocytes. We measured the selected inflammatory molecules in the supernatant collected from the control and the infected astrocytes after 48hpi. We observed that inhibition of TLR3 caused a significant decrease in ZIKV-induced IFN- $\beta$, RANTES, IP-10 and IL-6 secretion by the Puerto Rican strain (PRVABC59) in astrocytes (Figure 27). On the other hand, exposure with the TLR3 agonist, Poly I: C significantly increased the secretion of IFN- $\beta$, RANTES, and IP-10 in the supernatant of ZIKV-infected astrocytes (Figure 27).

To confirm whether the role of TLR3 signaling in ZIKV infection is limited to astrocytes or the same applies to other glial cells, we further examined its role in human microglia by using genetic silencing approach. We knocked down TLR3 with siRNA in microglia followed by infection with three different strains of ZIKV. A decrease in viral titers and secretion of inflammatory molecules was observed in human microglia after 
downregulation of TLR3 (Figure 28A-D), suggesting that TLR3 regulates ZIKV replication as well as ZIKV-induced secretion of inflammatory molecules in the glial cells.

We confirmed downregulation of the entire TLR3 signaling pathway with TLR3 knockdown in astrocytes exposed to ZIKV infection. TLR3 knockdown in astrocytes reverted ZIKV-induced upregulation of the adaptor protein TICAM1, transcription factor IRF3 and the alternative adaptor protein MyD88 (Figure 29). The data suggest that activation of the TLR3 signaling pathway is involved in ZIKV replication and associated neuropathologies.
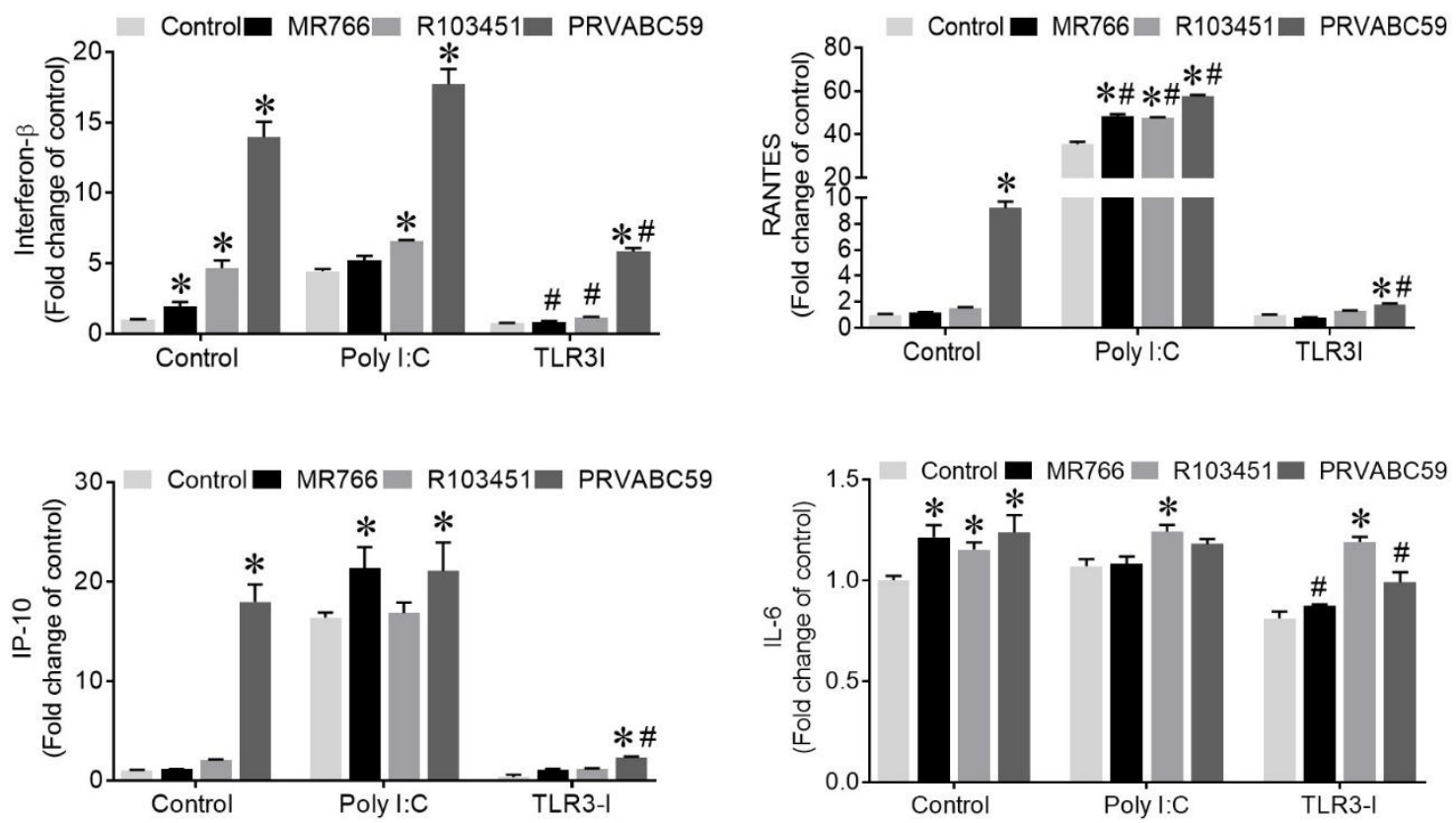

Figure 27. Silencing of TLR3 reduces ZIKV-induced antiviral and inflammatory molecules secretion.

Human astrocytes were infected with three different strains of ZIKV (MR766, R103451, and PRVABC59) at an MOI of 0.1. Sets of the experiment included astrocytes treated with $5 \mu \mathrm{g} / \mathrm{mL}$ of Poly I: C (TLR3 agonist), or with TLR3 silenced with siRNA or inhibited with a thiophenecarboxamidopropionate compound (TLR3/dsRNA complex inhibitor). Secretion of inflammatory molecules (IFN- $\beta$, IP-10, RANTES, and Il-6) was measured by ELISA from the supernatants collected at indicated time points. Data are presented as mean \pm SEM from 3 independent experiments and analyzed using two-way ANOVA with Bonferroni post hoc correction. * $\mathrm{p}<0.05 \mathrm{Vs}$ Control, \# $\mathrm{p}<0.05 \mathrm{Vs}$ ZIKV alone. 
A
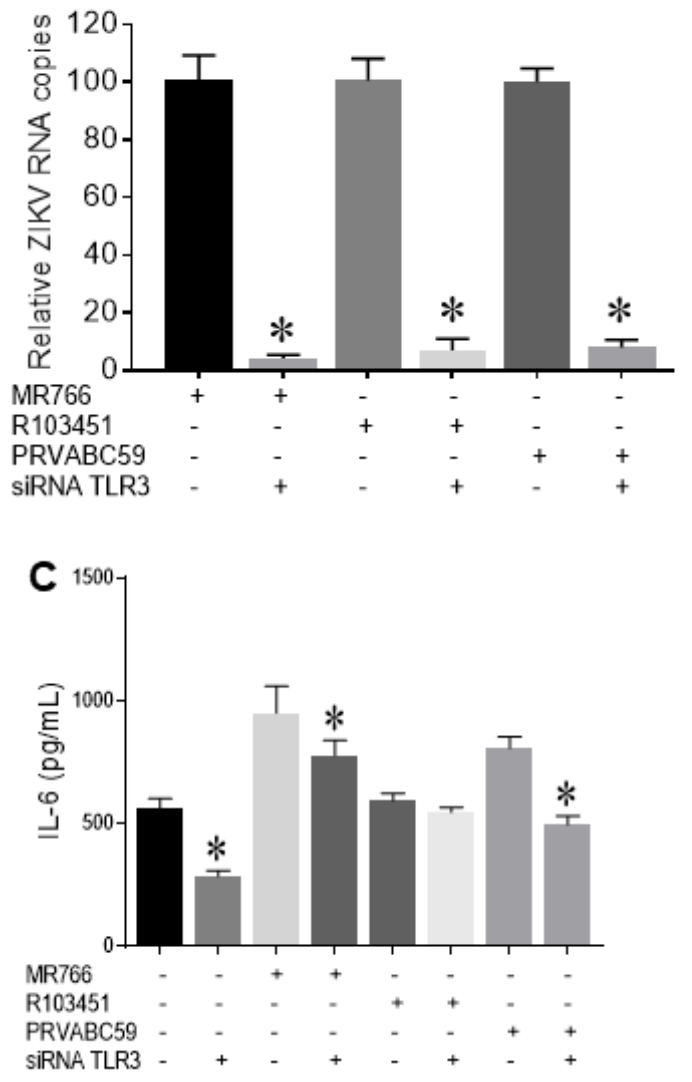
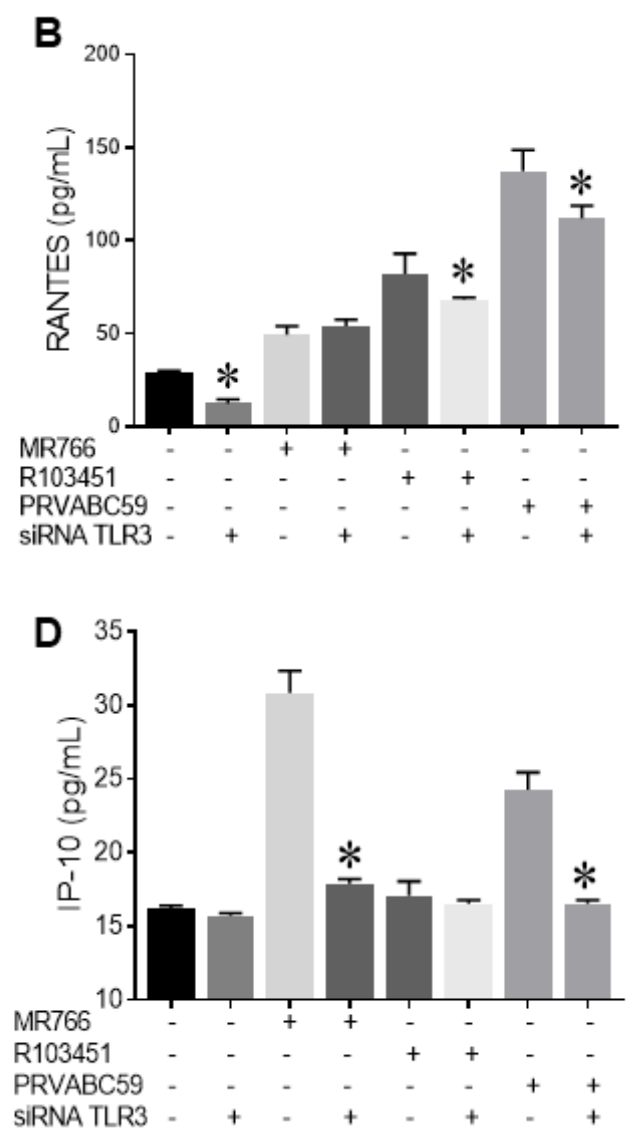

Figure 28. TLR3 regulates ZIKV replication and inflammatory response in human microglia.

(A) ZIKV titers measured by RT-PCR after 48hpi and TLR3 silencing. (B-D) Inflammatory molecules measured by ELISA after 48 hpi with or without siRNA against TLR3. Mock (PBS) infected cells were used as a control and the infection dose of ZIKV was at an MOI of 0.1. Data are presented as mean \pm SEM from 3 independent experiments and analyzed using one way ANOVA with Bonferroni post hoc correction. *p $<0.05$ Vs ZIKV. 

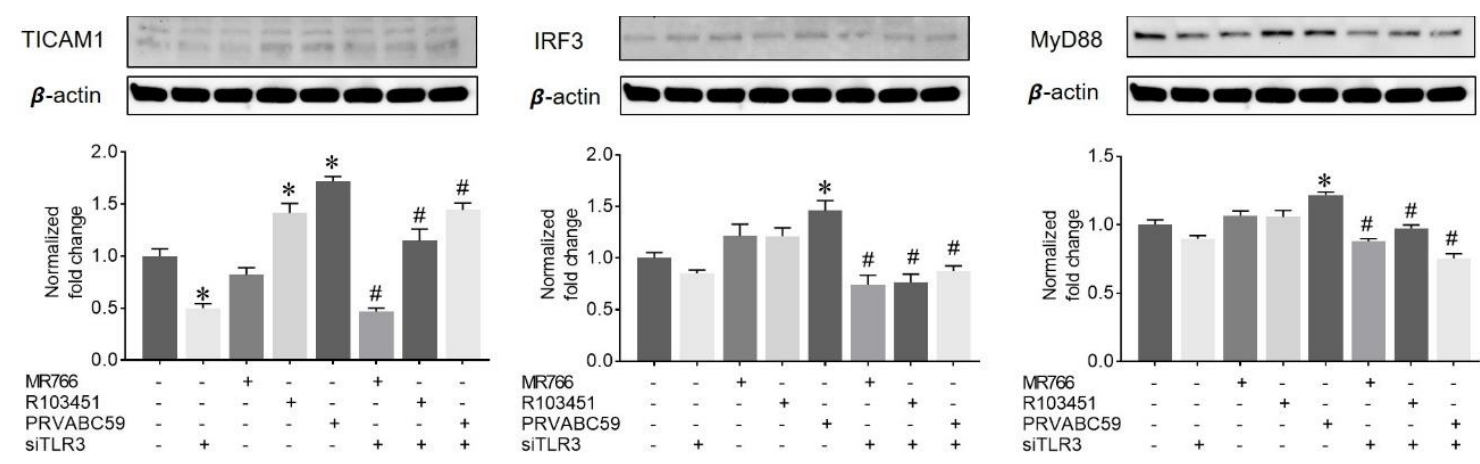

Figure 29. Silencing of TLR3 reverts ZIKV-induced upregulation of downstream signaling molecules.

TLR3 in the human astrocytes was knocked down by siRNA against TLR3 using lipofectamine 2000 transfection reagent. The cells (both knocked down and parental astrocytes) were infected with ZIKV at MOI of 0.1 and the protein expression of TICAM1, IRF3 and MyD88 were measured by immunoblotting after 48 hpi. Data are presented as mean \pm SEM from 3 independent experiments and analyzed using one way ANOVA with Bonferroni post hoc correction. * $\mathrm{p}<0.05$ Vs Control, \# p <0.05 Vs ZIKV alone.

\subsection{Discussion}

Once viral genomes are released into the target cells subsequently after entry, the flavivirus replication complex is assembled in close vicinity to the ER membranes. Following viral replication and processing of the polyprotein, packaging occurs on the surface of the ER and resulting immature virions are translocated to the Golgi complex where furin-mediated cleavage of prM to $\mathrm{M}$ generates mature virus which is released from the cells by exocytosis [23]. However, the host defense mechanisms within the cell create an adverse environment for the virus, and the virus has to evade or manipulate the cellular response for their survival and replication [24]. Of those defense mechanisms, the TLR pathway and the ER stress-related pathways are two important innate defense mechanisms operated within the cells to create an antiviral state and minimize the effects of viral toxicity $[4,25]$. 
In the current study, we reported that ZIKV induces ER stress and activates UPR. In line with our study, extensive rearrangement of ER membrane and collapse of ER cisternae juxtaposed with viral particles and virus-induced vesicles has been recorded by others using electron tomography, indicating that ZIKV replicates in the vicinity of the ER membranes [26]. We show that ZIKV mediated UPR activation mainly involves activation and phosphorylation of PERK, though minor activations in IRE-1 $\alpha$ and ATF-6 $\alpha$ was noted depending on the viral strains. We also reported that siRNA mediated silencing of PERK showed a slight but significant decrease in ZIKV replication. Our findings correlate with a previous study showing a reversal of ZIKV mediated neurogenic effects in mouse embryonic brain with PERK inhibitor [7]. However, the minimal effects of PERK on ZIKV replication with no significant effects on the secretion of inflammatory molecules, suggest a limited role of the UPR pathway in ZIKV infection. ZIKV mediated inductions in ER stress and related pathways might be the consequences of viral infection and replication inside the cells and may not necessarily control viral replication and associated pathology.

Exploring for alternative pathway involved in ZIKV infection, we detected that TLR3 was induced by the Asian strains of ZIKV in astrocytes. Similar to our findings, previous studies have shown the upregulation of TLR3 by ZIKV in various cell types including human astrocytes, skin fibroblast and mouse neurospheres [12, 27, 28]. TLR3 which recognizes viral dsRNA has an important role in flaviviral replication and associated pathology [22]. We also reported that silencing or pharmacological inhibition of TLR3 caused a significant decrease in RNA copies of both Asian and African strains of ZIKV with correlated decreases in the viral-induced inflammatory response, supporting the role 
of TLR3 in ZIKV replication and associated inflammatory immune response, irrespective of the strains.

Recently, a study reported that ZIKV-mediated over-activation of TLR3 leads to the depletion of neural progenitor cells in human cerebral organoids [12, 29], further validating our findings. Besides ZIKV infection, the role of TLR3 has already been reported for the closely related viruses such as WNV and DENV. TLR3 knock out mice are reported to be less vulnerable to severe WNV infection, cytokine production, neuronal injury and viral replication in the brain [22]. However, a protective role of TLR3 in WNV and DENV infection has also been reported by other groups [30, 31]. Studies have shown that TLR3 modulates virus permissiveness, replication, disease severity, immunogenicity in various other viruses including DENV, WNV, Influenza A virus, HIV-1, Herpes simplex, suggesting that TLR3 function is not specific to ZIKV [31-35].

TLR3 signaling leads to the activation of IRF3 which induces type 1 IFN responses, which is a primary antiviral defense strategy of the body. However, studies have shown that ZIKV nonstructural proteins disrupt the JAK-STAT signaling pathway to evade the type I IFN effector functions. Therefore, despite the induction of type 1 IFN production by activated TLR3, ZIKV replication might not be controlled. However, TLR3 mediated NF$\kappa \mathrm{B}$ activation and the resulting exaggerated, and persistent secretion of inflammatory molecules may facilitate neuroinflammation leading to neuropathologies associated with ZIKV. Differential levels of activation of NF- $\kappa B$ might also explain the differential levels of secretion of inflammatory molecules by the three spatiotemporally different ZIKV strains reported in chapter 2 . NF- $\kappa$ B is also reported to repress the expression of DDIT3, possibly limiting the DNA repair mechanism following ER stress [36, 37]. Silencing of 
TLR3 downregulates the entire downstream signaling pathway that is induced by TLR3, which is supported by a decrease in TICAM1 and IRF3 protein expression. Though TLR3 signaling is generally independent of MyD88, which was downregulated upon silencing of TLR3, a possible explanation might be siTLR3 suppressed the viral titers to the levels which may not be sufficient to induce MyD88.

In summary, ZIKV strains differentially activated the TLR pathway regulating the host innate immune defense. Attenuating TLR3 caused a decrease in viral replication that correlated with the decreased amount of in viral replication, making TLR3 a potential target for ZIKV therapeutic approach.

\section{References}

[1] Tang H, Hammack C, Ogden SC, Wen Z, Qian X, Li Y, et al. Zika Virus Infects Human Cortical Neural Precursors and Attenuates Their Growth. Cell stem cell. 2016;18(5):587-90.

[2] Gillespie LK, Hoenen A, Morgan G, Mackenzie JM. The endoplasmic reticulum provides the membrane platform for biogenesis of the flavivirus replication complex. J Virol. 2010;84(20):10438-47.

[3] Heaton NS, Perera R, Berger KL, Khadka S, Lacount DJ, Kuhn RJ, et al. Dengue virus nonstructural protein 3 redistributes fatty acid synthase to sites of viral replication and increases cellular fatty acid synthesis. Proc Natl Acad Sci U S A. 2010;107(40):17345-50.

[4] Blázquez AB, Escribano-Romero E, Merino-Ramos T, Saiz JC, Martín-Acebes MA. Stress responses in flavivirus-infected cells: activation of unfolded protein response and autophagy. Front Microbiol. 2014;5.

[5] Hetz C. The unfolded protein response: controlling cell fate decisions under ER stress and beyond. Nature Reviews Molecular Cell Biology. 2012;13:89.

[6] Liu CY, Schroder M, Kaufman RJ. Ligand-independent dimerization activates the stress response kinases IRE1 and PERK in the lumen of the endoplasmic reticulum. J Biol Chem. 2000;275(32):24881-5. 
[7] Gladwyn-Ng I, Cordón-Barris L, Alfano C, Creppe C, Couderc T, Morelli G, et al. Stress-induced unfolded protein response contributes to Zika virus-associated microcephaly. Nature Neuroscience. 2018;21(1):63-71.

[8] Routhu NK, Byrareddy SN. Host-Virus Interaction of ZIKA Virus in Modulating Disease Pathogenesis. Journal of Neuroimmune Pharmacology. 2017;12(2):21932.

[9] Marinelli C, Di Liddo R, Facci L, Bertalot T, Conconi MT, Zusso M, et al. Ligand engagement of Toll-like receptors regulates their expression in cortical microglia and astrocytes. J Neuroinflammation. 2015;12.

[10] Garcia M, Wehbe M, Lévêque N, Bodet C. Skin innate immune response to flaviviral infection. European Cytokine Network. 2017;28(2):41-51.

[11] Koyama S, Ishii KJ, Coban C, Akira S. Innate immune response to viral infection. Cytokine. 2008;43(3):336-41.

[12] Dang J, Tiwari SK, Lichinchi G, Qin Y, Patil VS, Eroshkin AM, et al. Zika Virus Depletes Neural Progenitors in Human Cerebral Organoids through Activation of the Innate Immune Receptor TLR3. Cell stem cell. 2016;19(2):258-65.

[13] Fukunaga K, Shioda N. Pathophysiological Relevance of Forkhead Transcription Factors in Brain Ischemia. In: Maiese K, editor. Forkhead Transcription Factors: Vital Elements in Biology and Medicine. New York, NY: Springer New York; 2010. p. 130-42.

[14] Braaten D, Luban J. Cyclophilin A regulates HIV-1 infectivity, as demonstrated by gene targeting in human T cells. The EMBO Journal. 2001;20(6):1300-9.

[15] Neidhart S, Antonsson B, Gillieron C, Vilbois F, Grenningloh G, Arkinstall S. cJun N-terminal kinase-3 (JNK3)/stress-activated protein kinase-beta (SAPKbeta) binds and phosphorylates the neuronal microtubule regulator SCG10. FEBS Lett. 2001;508(2):259-64.

[16] Dong X-Y, Tang S-Q, Chen J-D. Dual functions of Insig proteins in cholesterol homeostasis. Lipids in Health and Disease. 2012;11(1):173.

[17] Darling NJ, Cook SJ. The role of MAPK signalling pathways in the response to endoplasmic reticulum stress. Biochimica et Biophysica Acta (BBA) - Molecular Cell Research. 2014;1843(10):2150-63.

[18] Besnard a, Galan B, Vanhoutte p, Caboche J. Elk-1 a Transcription Factor with Multiple Facets in the Brain. Frontiers in Neuroscience. 2011;5(35). 
[19] Chang M, Jin W, Sun S-C. Peli1 facilitates TRIF-dependent Toll-like receptor signaling and proinflammatory cytokine production. Nature Immunology. 2009;10:1089.

[20] Li Q, Verma IM. NF-kappaB regulation in the immune system. Nat Rev Immunol. 2002;2(10):725-34.

[21] Karin M, Cao Y, Greten FR, Li ZW. NF-kappaB in cancer: from innocent bystander to major culprit. Nature reviews Cancer. 2002;2(4):301-10.

[22] Wang T, Town T, Alexopoulou L, Anderson JF, Fikrig E, Flavell RA. Toll-like receptor 3 mediates West Nile virus entry into the brain causing lethal encephalitis. Nature Medicine. 2004;10(12):1366.

[23] Sager G, Gabaglio S, Sztul E, Belov GA. Role of Host Cell Secretory Machinery in Zika Virus Life Cycle. Viruses. 2018;10(10):559.

[24] Barber GN. Host defense, viruses and apoptosis. Cell death and differentiation. 2001;8(2):113-26.

[25] Thompson MR, Kaminski JJ, Kurt-Jones EA, Fitzgerald KA. Pattern Recognition Receptors and the Innate Immune Response to Viral Infection. Viruses. 2011;3(6):920-40.

[26] Stefanik M, Formanova P, Bily T, Vancova M, Eyer L, Palus M, et al. Characterisation of Zika virus infection in primary human astrocytes. BMC Neuroscience. 2018;19(1):5.

[27] Hamel R, Dejarnac O, Wichit S, Ekchariyawat P, Neyret A, Luplertlop N, et al. Biology of Zika Virus Infection in Human Skin Cells. Journal of Virology. 2015;89(17):8880.

[28] Hamel R, Ferraris P, Wichit S, Diop F, Talignani L, Pompon J, et al. African and Asian Zika virus strains differentially induce early antiviral responses in primary human astrocytes. Infection, Genetics and Evolution. 2017;49:134-7.

[29] Marziale M. The Mechanism of Nervous System Damage by Zika Virus and the Role of Toll-like 3 Receptors â $\square$ " Proceedings of the Texas A\&M Medical Student Grand Rounds. Procedings of the Texas A\&M Medical Student Grand rounds. 2017; OCt 2017.

[30] Daffis S, Samuel MA, Suthar MS, Jr. MG, Diamond MS. Toll-Like Receptor 3 Has a Protective Role against West Nile Virus Infection. Journal of Virology. 2008. 
[31] Tsai YT, Chang SY, Lee CN, Kao CL. Human TLR3 recognizes dengue virus and modulates viral replication in vitro. Cellular microbiology. 2009;11(4):604-15.

[32] Wang T, Town T, Alexopoulou L, Anderson JF, Fikrig E, Flavell RA. Toll-like receptor 3 mediates West Nile virus entry into the brain causing lethal encephalitis. Nature medicine. 2004;10(12):1366-73.

[33] Breckpot K, Escors D, Arce F, Lopes L, Karwacz K, Van Lint S, et al. HIV-1 Lentiviral Vector Immunogenicity Is Mediated by Toll-Like Receptor 3 (TLR3) and TLR7. Journal of Virology. 2010;84(11):5627.

[34] Goffic RL, Balloy V, Lagranderie M, Alexopoulou L, Escriou N, Flavell R, et al. Detrimental Contribution of the Toll-Like Receptor (TLR)3 to Influenza A VirusInduced Acute Pneumonia. PLOS Pathogens. 2006;2(6):e53.

[35] Reinert LS, Harder L, Holm CK, Iversen MB, Horan KA, Dagnæs-Hansen F, et al. TLR3 deficiency renders astrocytes permissive to herpes simplex virus infection and facilitates establishment of CNS infection in mice. The Journal of Clinical Investigation. 2012;122(4):1368-76.

[36] Nozaki S, Sledge GW, Jr., Nakshatri H. Repression of GADD153/CHOP by NFkappaB: a possible cellular defense against endoplasmic reticulum stress-induced cell death. Oncogene. 2001;20(17):2178-85.

[37] Willy JA, Young SK, Stevens JL, Masuoka HC, Wek RC. CHOP links endoplasmic reticulum stress to NF- $\kappa \mathrm{B}$ activation in the pathogenesis of nonalcoholic steatohepatitis. Molecular Biology of the Cell. 2015;26(12):2190204. 


\section{CHAPTER 5: ROLE OF AUTOPHAGY PATHWAY IN ZIKV INFECTION AND}

\section{ASSOCIATED NEUROPATHOLOGY}

\subsection{Introduction}

Autophagy is a complex process of lysosomal degradation of unfolded or misfolded proteins, intracellular pathogens, and dysfunctional organelles via the formation of a double membrane structure known as an autophagosome. Autophagy pathway which involves more than 30 autophagy-related (ATG) proteins [1], is crucial for multiple cellular activities such as cellular development and differentiation [2], innate and adaptive immunity [3, 4], and programmed cell death (type II) $[5,6]$. The autophagosome fuses with endocytic and lysosomal compartments to form autoendosome or autolysosome for subsequent degradation [7].

As a part of the host innate stress response, autophagy is frequently a by-product of viral infection-triggered cellular stress. During viral infection, autophagy is activated by the innate immune system to degrade and dispose of invading viruses. A selective form of autophagy referred to as xenophagy is directly involved in the recognition and lysosomal degradation of the intracellular microorganisms via autophagosomes [8]. Autophagy pathway also facilitates viral antigen presentation leading to the induction

of adaptive immune responses. However, the membrane-bound protected platform of the autophagosome along with the autophagy generated metabolites is hijacked by many viruses for their shelter and replication $[8,9]$. Viruses have evolved various strategies to combat or exploit the autophagy pathway for their replication [8].

Several viruses including Poliovirus, hepatitis C virus, Dengue virus, and coronaviruses are known to subvert the autophagy pathway into a pro-viral mechanism. 
However, the exact mechanism by which autophagy contributes to viral infection is still unclear [9]. In case of ZIKV infection, induction of autophagy is reported by others in different cell types including HeLa cells, fibroblast, fNSCs and placental trophoblastic cells potentially mediated by NS4A and NS4B proteins through inhibition of the AktmTOR signaling pathway $[10,11]$. Inhibiting the autophagy pathway with 3methyladenine (3-MA), chloroquine or gene silencing targeting the autophagy-related gene (ATG-3 and ATG-5) resulted in a decrease in viral replication in various cell types. While, induction of autophagy with rapamycin promoted ZIKV replication $[10,11]$. These findings present preliminary evidence that ZIKV infection induces autophagy pathway potentially leading to increased ZIKV replication and viral load.

In the current study, we reported that ZIKV induces the autophagy pathway in human primary astrocytes. However, autophagy pathway is not directly involved in ZIKV replication in astrocytes. Nevertheless, genetic and pharmacological inhibition of autophagy or the autophagy protein, Beclin1, resulted in exacerbation of ZIKV associated inflammatory responses in the astrocytes. These data indicate that unlike other viruses, ZIKV has not evolved mechanism to subvert the autophagy pathway in order to facilitate its replication.

\subsection{Methodology}

\subsubsection{Reagents}

Rapamycin was purchased from Sigma-Aldrich (St. Louis, MO, USA) and used at a concentration of $2.5 \mu \mathrm{M}$. 3-methyladenine (3-MA) and chloroquine (CQ) were used at a concentration of $1 \mu \mathrm{M}$ and $40 \mu \mathrm{M}$, respectively. The concentrations were based on the doseresponse viability assay performed in our previous studies [12, 13]. 


\subsubsection{Immunoblotting}

Primary antibodies against Beclin1 (Cat. ab62557 (1:1500), Abcam, Cambridge, MA, USA), LC3B (Cat. 12741 (1:1000), Cell Signaling, Danvers, MA, USA), p62/SQSTM1 (Cat. ab91526 (1:2000), Abcam, Cambridge, MA, USA), ZIKV anti-E (Cat. GTX133314, (1:1000) Genetex, Irvine, CA, USA), were followed by incubation with a respective secondary antibody conjugated to horseradish peroxidase (Millipore, Billerica, MA, USA) used at specified dilution.

\subsubsection{Cell-based LC3B assay for autophagosome and autolysosome}

LC3B localization analysis for human astrocytes was performed by using Premo $^{\text {TM }}$ Autophagy GFP-RFP-LC3B cell-based assay (Life Technologies, Cat. No. P36241). The cells were plated at the desired density, allowed to adhere to and infected with ZIKV. After infection, LC3B sensor was added at a concentration of 30 particles per cells (PPC) and incubated overnight. DAPI was used to label cell nuclei. Fluorescently labeled cells were visualized using an inverted fluorescent microscope (Zeiss). Yellow puncta representing autophagosome and red puncta representing autolysosome were counted and expressed as number per cells.

\subsubsection{Silencing of Beclin1 in human astrocytes}

Human primary astrocytes were transfected with siRNA against Beclin1 using Lipofectamine 2000 (Invitrogen, Carlsbad, CA, USA) or Fugene HD (Promega, Madison, WI, USA) for Beclin1 knockdown. siRNA and transfection reagent (ratio, 1:2) were preincubated for $20 \mathrm{~min}$ in OptiMEM medium before addition to the seeded cells. Knockdown of targets was confirmed in cell lysates by western blot for the protein expression of Beclin1. CRISPR-cas9 method of silencing was also utilized for enhanced silencing of 
Beclin1 in human astrocytes. Guide RNA (gRNA) for BECN1 and Cas9 enzyme were mixed in the ratio of 1.3:1 and incubated at room temperature for 5 minutes to make RNPcomplex, followed by incubation with transfusion reagent (Fugene HD, Promega, Madison, WI, USA) for 20 minutes. The resulting RNP-transfection solution was added to the seeded cells and incubated for at least 24 hours before ZIKV infection.

\subsubsection{Statistical analysis}

Results are reported as mean \pm SEM of 3-6 independent experiments each with more than three samples. Data were analyzed using analysis of variance (ANOVA) followed by appropriate post hoc test for multiple comparisons (Graph Pad Software, Inc., La Jolla, CA, USA). An alpha level (p-value) of $<0.05$ was considered significant.

\subsection{Results}

\subsubsection{Autophagy is induced in ZIKV-infected human astrocytes}

Viral infection is known to activate the autophagy pathway, which can be an attractive target for various viruses $[14,15]$. Induction of autophagosome formation in various cell types infected with different strains of ZIKV has already been reported [16, 17]. However, the effect of ZIKV on glial cell-autophagy is not reported yet. To this end, we measured the protein expression of key proteins involved in the autophagy pathway in human astrocytes with or without ZIKV infection (Figure 30). Infection of astrocytes with MR766, R103451, and PRVABBC59 strains of ZIKV showed about 1.3, 1.6 and 1.9-fold increase respectively, in the expression of Beclin1, a major protein involved in the initiation of autophagosome formation. LC3B-II, a marker for autophagosome formation [12, 18] was also increased by about 2-fold, while p62/SQSTM1, a ubiquitin-binding scaffold protein that facilitates binding of ubiquitinated protein to autophagosome and a marker for 
autophagosome degradation, was upregulated by about 1.5 fold (Figure 30) in astrocytes infected with all three strains of ZIKV.
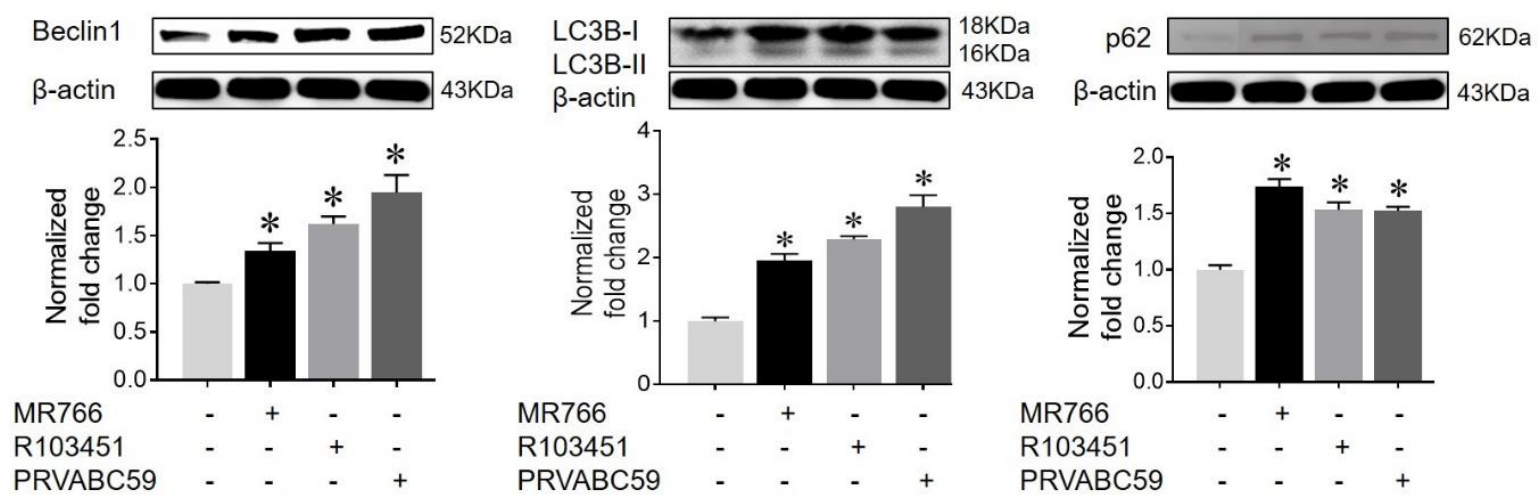

Figure 30. ZIKV induces autophagosome formation and blocks autophagosomelysosome fusion.

Human astrocytes were infected with three different strains of ZIKV (MOI = 0.1), cell lysates collected at $48 \mathrm{hpi}$ and protein expression of Beclin1, LC3B and p62/SQSTM1 were measured by western blot. Data are presented as mean \pm SEM from 3 independent experiments and analyzed using one-way ANOVA with Bonferroni post hoc correction. *p $<0.05$ Vs Control.

Analysis of the autophagy pathway using a cell-based assay that utilizes the tandem mRFP-GFP tagged LC3 reporter plasmid for fluorescence analysis (Figure 31A-C) showed similar results with upregulation in autophagosome formation in astrocytes infected with each of the ZIKV strain. The tandem mRFP-GFP LC3 assay employs the differential $\mathrm{pH}$ stability of green fluorescent protein (GFP) and a red fluorescent protein (RFP) in an acidic environment of the lysosome. We measured a 2-fold increase in autophagosome formation in astrocytes infected with R103451 and PRVABC59 strains of $\mathrm{ZIKV}$, as indicated by the yellow puncta (Figure 31A and $\mathrm{B}$ ) and a decrease in the maturation or lysosomal pathway in cells infected with each of the strains (Figure 31A and C), as detected by a decrease in red puncta. Overall, we showed activation of the autophagy pathway in cells infected with each strain of ZIKV. The western blot and cell-based assay data imply that infection by ZIKV induces the initial activation and maturation stages of 
the autophagy pathway, yet, blocks the autophagosome-lysosome fusion and degradation of the cargo. This, in turn, prompted us to further examine the role of autophagy pathway in ZIKV infectivity and pathology in human astrocytes.
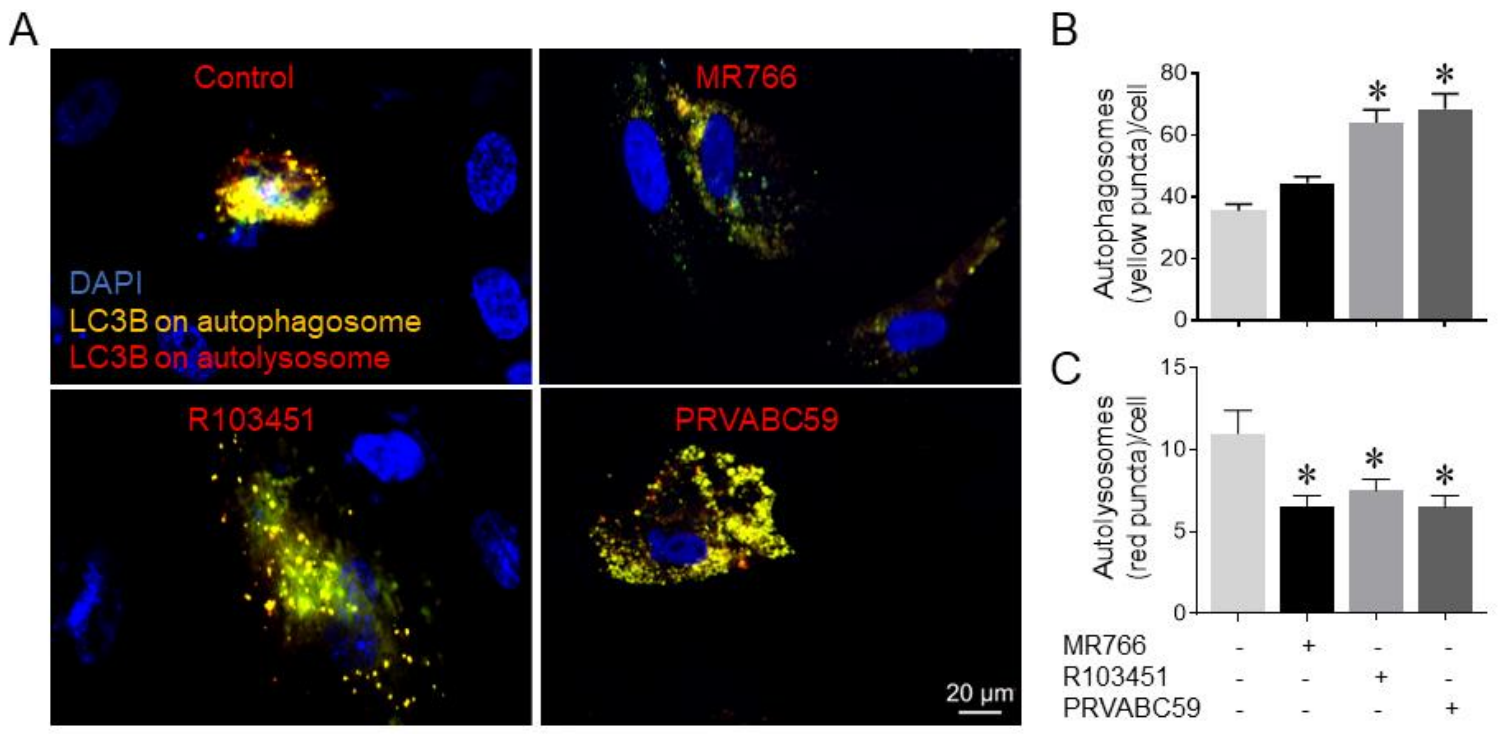

Figure 31. ZIKV mediated autophagosome induction and subsequent blockade in autophagosome-lysosome fusion confirmed by the cell-based assay.

(A) Representative images of uninfected and ZIKV (three strains) infected human astrocytes shows yellow and red LC3B puncta as determined by a cell-based assay for LC3B. (B and C) The numbers of yellow and red puncta from the cell-based assay were counted per cells. Presence of both green and red (yellow) fluorescence indicates autophagosome, while red fluorescence only indicates autolysosome. Data are presented as mean \pm SEM from 3 independent experiments and analyzed using one-way ANOVA with Bonferroni post hoc correction. *p $<0.05$ Vs Control.

\subsubsection{Modulation of the autophagy pathway has minimal effect on ZIKV infection}

\section{in human astrocytes}

Next, we explored the role of autophagy in mediating ZIKV replication and associated pathogenesis in glial cells by using pharmacological and genetic approaches to modulate the autophagy pathway in ZIKV-infected human astrocytes. Cell supernatants were collected at $48 \mathrm{hpi}$, and RNA titer (indicative of viral replication) of each viral strain was determined by RT-PCR and confirmed by the plaque-forming assay. Induction of the 
autophagy pathway with rapamycin, an autophagy activator, caused a slight but insignificant increase in the viral titer of R103451. No further changes were detected in the viral titer of MR766 and PRVABBC59 in the astrocytes treated with rapamycin. Blocking the formation of autophagosome with 3-MA did not affect ZIKV replication irrespective of the viral strains. Exposure with chloroquine (CQ), a lysosomotropic agent that inhibits lysosomal degradation, caused a slight but insignificant decrease in the replication of PRVABC59 in human astrocytes (Figure 32).

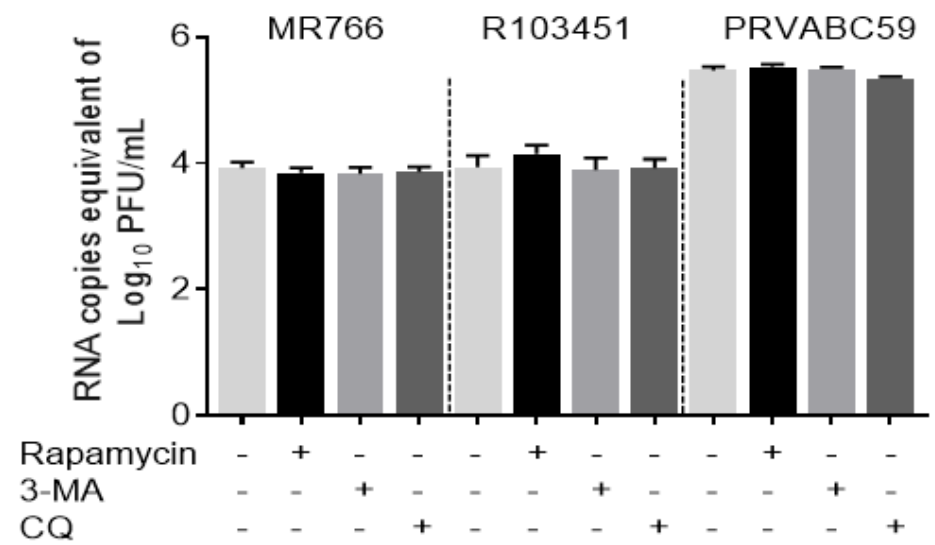

Figure 32. Modulation of Autophagy pathway shows no significant changes in viral replication.

The autophagy pathway was modulated before ZIKV infection either by using pharmacological inducer (rapamycin) or inhibitors (3-MA or chloroquine), and viral titers were measured by RT-PCR using cell supernatants collected after infection with three different strains of ZIKV and indicated treatments. Data are presented as mean \pm SEM from 3 independent experiments and analyzed using one-way ANOVA with Bonferroni post hoc correction.

We detected a significant decrease in MR766-induced secretion of IFN- $\beta$ with similar trends in other strains with rapamycin indicating a probable role of the mTOR signaling pathway in IFN response. Exposure to 3-MA did not affect ZIKV-induced IFN$\beta$ secretion in the astrocytes. However, exposure with chloroquine caused a significant 
increase in R103451- and PRVABC59-induced secretion of IFN- $\beta$ (Figure 33A). Similarly, exposure to CQ enhanced the ZIKV-induced secretion of IP-10 in astrocytes infected with each of the viral strains (Figure 33B). Except for the decrease in PRVABC59-induced RANTES secretion, 3-MA had no significant effect on ZIKV mediated inflammatory molecules (Figure 33C). There are minor changes in ZIKVinduced secretion of IL-6 with rapamycin, 3-MA and CQ (Figure 33D).
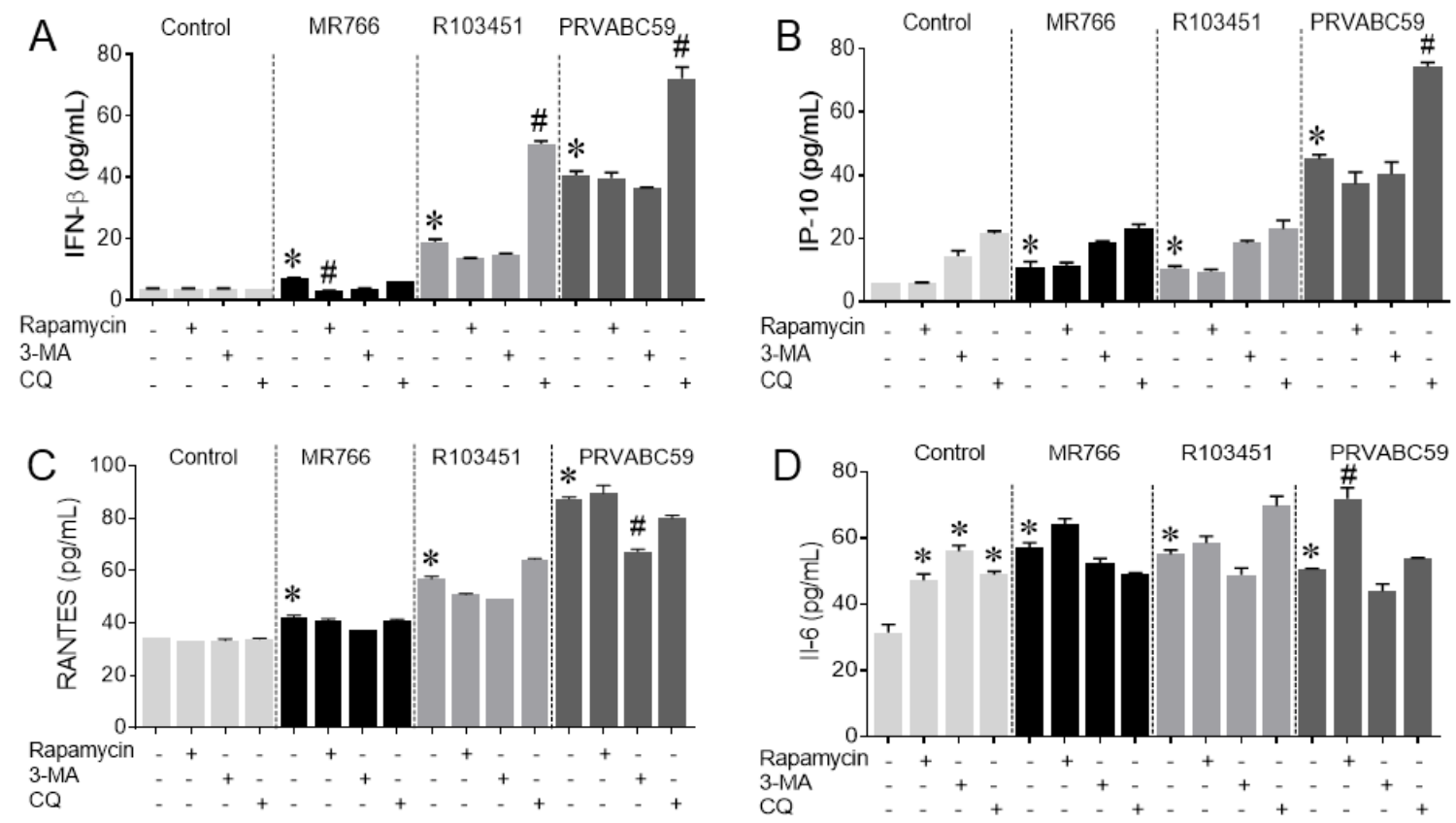

Figure 33. Modulation of Autophagy pathway shows a minor effect in ZIKV mediated secretion of inflammatory molecules.

Human astrocytes exposed either to rapamycin or 3-MA or chloroquine were infected with three different strains of ZIKV. Cell supernatants collected after 48 hpi were used to measure IFN- $\beta$, IP-10, RANTES, and IL- 6 by ELISA. Data are presented as mean \pm SEM from 3 independent experiments and analyzed using one-way ANOVA with Bonferroni post hoc correction. *p $<0.05$ Vs Control, \# p <0.05 Vs ZIKV alone.

\subsubsection{Silencing of Beclin1 exacerbates ZIKV induced inflammatory responses in}

\section{human astrocytes without affecting ZIKV replication}

Next, we examined the role of Beclin1 in the pathology of ZIKV since we have previously reported that Beclin1 regulates the replication and viral-induced neuro- 
inflammation in glial cells infected with the Human Immunodeficiency virus [19-21]. We silenced the expression of Beclin1 by CRISPR/cas9 and siRNA against Beclin1, and the knockdown was confirmed by western blot (Figure 34A). Although, all three strains of ZIKV induced the expression of Beclin1 (Figure 30), silencing with CRISPR-Cas9 did not affect the ZIKV replication in astrocytes (Figure 34B).
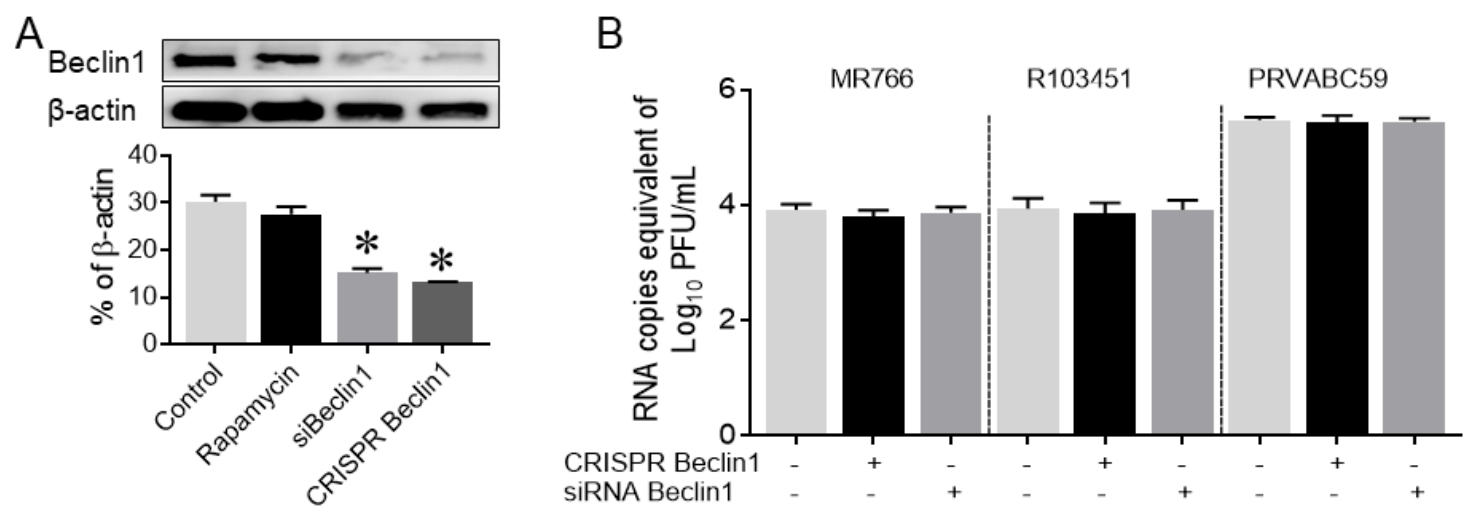

Figure 34. Silencing of Beclin1 does not affect the replication of ZIKV.

(A) Beclin1 in the human primary astrocytes was knocked down using small interfering RNA (siRNA) against Beclin1 or clustered regularly interspaced short palindromic repeats (CRISPER)/cas9 with guide RNA specific for Beclin1. (B) The astrocytes with or without Beclin1 knockdown was infected with three different strains of ZIKV, and viral RNA was measured in the supernatant collected at $48 \mathrm{hpi}$. Data are presented as mean \pm SEM from 3 independent experiments and analyzed using one-way ANOVA with Bonferroni post hoc correction. *p $<0.05$ Vs Control.

Similarly, Secretion of IFN- $\beta$ was not affected by Beclin1 silencing (both siRNA and CRISPR mediated) in human astrocytes (Figure 35A). However, silencing Beclin1 further enhanced ZIKV-induced secretion of inflammatory molecules, IP-10, RANTES, and IL-6 (Figure 35B-D). The effect of Beclin1 knockdown in the secretion of IP-10 was not very high (Figure 35B), but there was up to 7-fold increase in RANTES (Figure 35C), and up to 12-fold increase in IL-6 (Figure 35D) secretions in the astrocytes with Beclin1 knockdown. Moreover, silencing of Beclin1 caused increase secretion of RANTES and IL- 
6 even in uninfected astrocytes, indicating the anti-inflammatory or protective role of Beclin1.

Overall, the increase in the release of inflammatory molecules did not correlate with the viral titer in the astrocytes suggesting that ZIKV-induced inflammatory responses are not mediated by the autophagy pathway, but by Beclin1 via a different mechanism. The data further show that the basal level of Beclin1 protein is necessary to control the exaggerated secretion of cytokines and chemokines. However, our data do not rule out the possibility that silencing of Beclin1 itself may induce inflammatory molecules secretion in primary astrocytes.
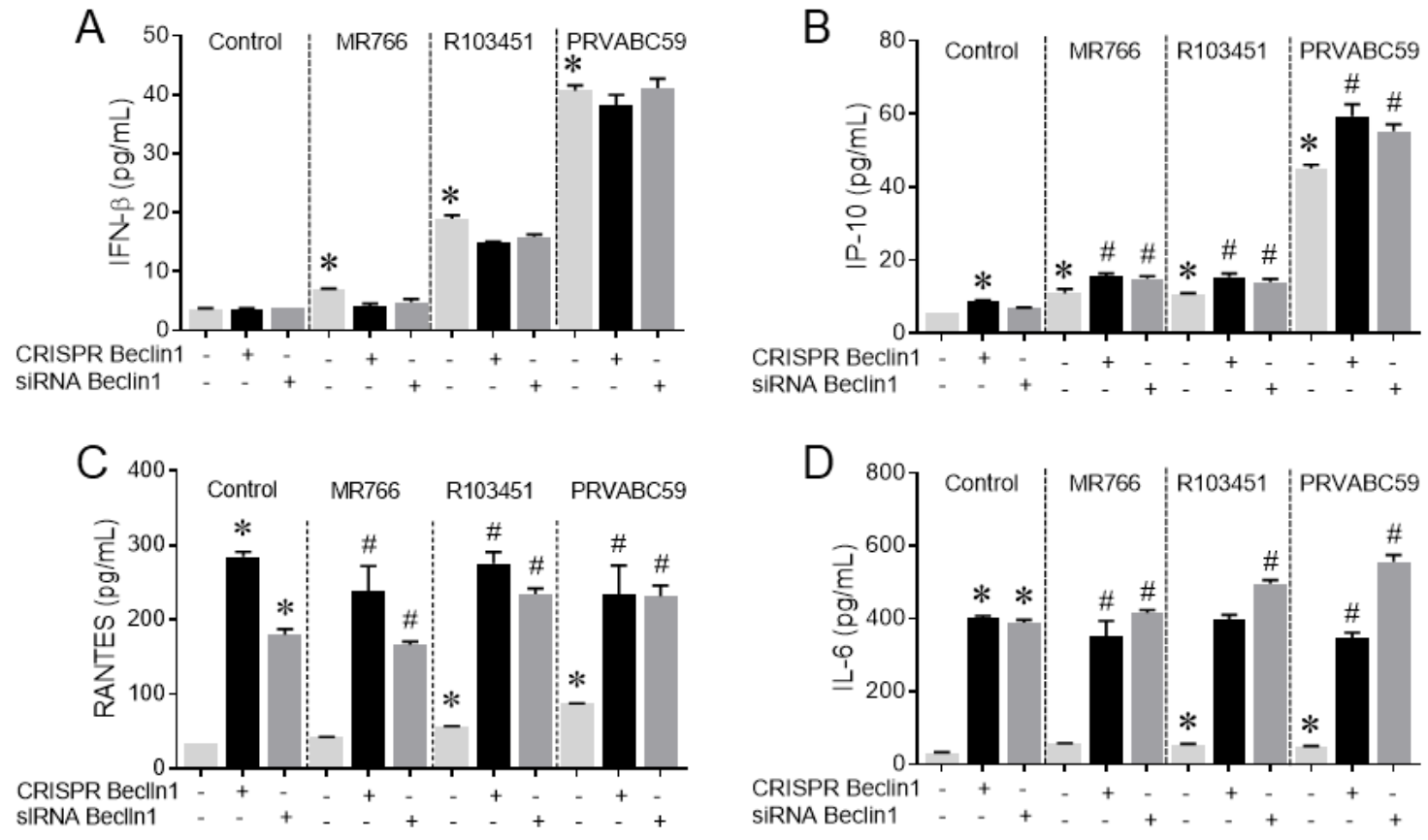

Figure 35. Silencing of Beclin1 exacerbates ZIKV-induced inflammatory responses in human astrocytes.

Beclin1 in the human primary astrocytes was knocked down using siRNA against Beclin1 or CRISPER /cas9 with guide RNA specific for Beclin1. The astrocytes with or without Beclin1 knockdown was infected with three different strains of ZIKV, and the indicated inflammatory molecules were measured in the supernatant collected at 48 hpi. Data are presented as mean \pm SEM from 3 independent experiments and analyzed using one-way 
ANOVA with Bonferroni post hoc correction. *p $<0.05$ Vs Control, \# $p<0.05$ Vs ZIKV alone.

\subsubsection{Induction of autophagy pathway by ZIKV is potentially mediated via the}

\section{TLR3 pathway}

Since the TLR signaling pathway is reported to induce the autophagy pathway [22] via adaptor proteins MyD88 or TICAM, we next investigated a potential link between TLR3 and the autophagy pathway in the context of ZIKV infection and pathology. Attenuation of TLR3 by genetic silencing resulted in a decrease in the expression levels of Beclin1 (Figure 36A) suggesting a potential interaction between the proteins involved in TLR signaling and autophagy pathway or inhibition of a common protein necessary for the induction of both the pathways. Interestingly, silencing TLR3 caused a further increase in the protein expression levels of p62/SQSTM1 (Figure 36B) suggesting the accumulation of autophagosomes. The accumulation of autophagosomes might result from the suppression of autophagy pathway added to the ZIKV-induced blockade in the completion of basal autophagy [23]. Overall, our data suggest that TLR3 mediates autophagy in ZIKVinfected astrocytes, although TLR3 mediated ZIKV replication may be independent of the autophagy pathway. 
A
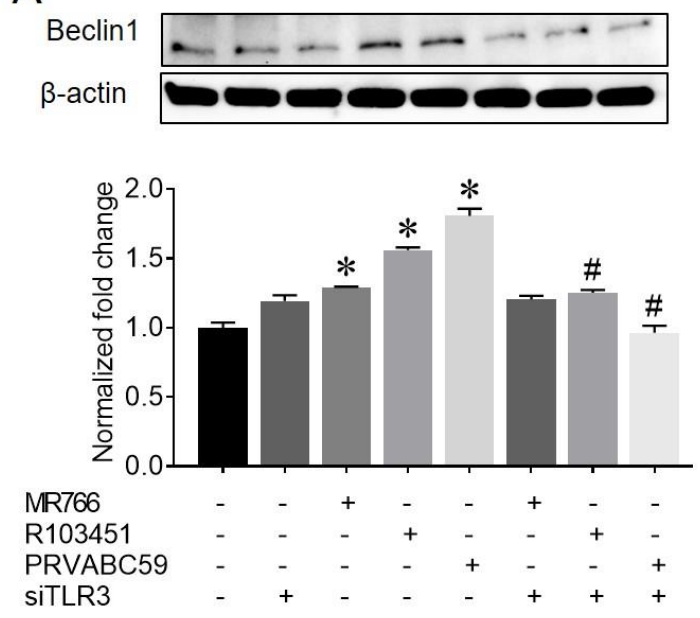

B
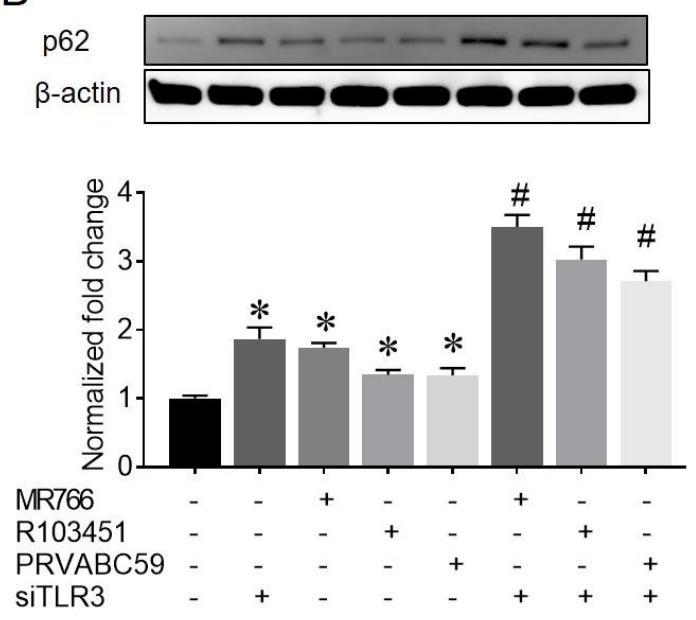

Figure 36. Silencing of TLR3 reverts ZIKV-mediated autophagy induction.

Protein expression of Beclin1 and p62 was measured by western blot at 48 hpi in TLR3 silenced astrocytes infected with three different strains of ZIKV. Data are presented as mean \pm SEM from 3 independent experiments and analyzed using one-way ANOVA with Bonferroni post hoc correction. ${ }^{*} \mathrm{p}<0.05$ Vs Control, \# $\mathrm{p}<0.05$ Vs ZIKV alone.

\subsection{Discussion}

Autophagy, as an innate defense mechanism, has the potential to restrict viral replication. However, viruses including flaviviruses evolve specific mechanisms to overcome autophagic degradation [24]. Our data showed that ZIKV induces the autophagy pathway with possible blockage of autophagic clearance in human astrocytes, irrespective of viral strains. Our findings are in concordance with what others have reported in different cells $[25,26]$. Since, astrocytes are the predominant cell types in the brain and are involved in diverse functions including maintenance of the homeostasis, enhancement of the myelination, the synapses and the blood-brain barrier, any impairment of the autophagy pathway in astrocytes may contribute to neuronal dysfunction.

However, modulation of the autophagy pathway did not affect ZIKV replication in human astrocytes, unlike the data reported by others using fNSCs, HeLa cells and MEF cells $[10,11]$. The possible explanation for the anomalies might be variations in the viral 
strains, passage numbers, and sources of the astrocytes, MOI of virus used and other factors or the effects are cell-type specific. However, we observed minor viral strain-specific changes in the secretion of inflammatory molecules by rapamycin, 3-MA or chloroquine exposed human astrocytes. MR766-induced IFN- $\beta$ secretion was decreased by rapamycin, while R103451-induced IFN- $\beta$ secretion was increased by chloroquine. Similarly, exposure to chloroquine enhanced the secretion of IP-10 induced by R103451 and PRVABC59 strains of ZIKV. These data indicate that the autophagy pathway is associated with ZIKV induced inflammatory responses without directly altering the viral replication. Silencing of Beclin1 caused further enhancement in the secretion of RANTES, IP-10, and IL-6, which may be related to any cellular mechanism involving Beclin1 other than the autophagy pathway.

Both the autophagy and the TLR3 pathways are important mediators of the innate immune response, and we report that ZIKV induces both the pathways. Activation of TLR3 was previously reported to induce the autophagy pathway [22, 27-29]. MyD88 and TICAM1, two adaptor proteins involved in the TLR signaling pathway can interact with the autophagy protein, Beclin1 with this interaction inhibiting the binding between Beclin1 and the anti-apoptotic protein, B-cell lymphoma 2 (BCL-2). Dissociation of Beclin1 from BCL-2 by activated MyD88 or TICAM1 may facilitate the induction of the autophagy pathway, while autophagic processing of viral RNA, in turn, may activate TLR3 signaling pathway [29]. A decrease in ZIKV-induced expression of Beclin1 by TLR3 silencing in the current study support that TLR3 pathway mediates autophagy pathway directly or indirectly via an unknown mechanism. Increase in p62/SQSTM1 with TLR3 silencing in ZIKV infected astrocytes might also suggest the role of p62/SQSTM1 to facilitate 
clearance of viral proteins via the ubiquitin-proteasomal system, while the autophagy pathway is downregulated [23].

Overall, we report that ZIKV strains differentially activated the autophagy, important mechanisms involved in regulating the host innate immune defense. Modulation of the autophagy pathway caused changes in ZIKV-induced inflammatory responses, which did not correlate with the viral titer released from human astrocytes. Autophagy pathway to some extent and Beclin1 play a protective role in ZIKV mediated inflammation. Together with the findings in chapter III, the data indicate that ZIKV-mediated induction of autophagy pathway is probably associated with the TLR3 signaling pathway.

\section{References}

[1] Ojha CR, Lapierre J, Rodriguez M, Dever SM, Zadeh MA, DeMarino C, et al. Interplay between Autophagy, Exosomes and HIV-1 Associated Neurological Disorders: New Insights for Diagnosis and Therapeutic Applications. Viruses. 2017;9(7).

[2] Tsukamoto S, Kuma A, Murakami M, Kishi C, Yamamoto A, Mizushima N. Autophagy is essential for preimplantation development of mouse embryos. Science. 2008;321(5885):117-20.

[3] Xu Y, Jagannath C, Liu XD, Sharafkhaneh A, Kolodziejska KE, Eissa NT. Tolllike receptor 4 is a sensor for autophagy associated with innate immunity. Immunity. 2007;27(1):135-44.

[4] Shibutani ST, Saitoh T, Nowag H, Munz C, Yoshimori T. Autophagy and autophagy-related proteins in the immune system. Nat Immunol. 2015;16(10):1014-24.

[5] Yu L, Alva A, Su H, Dutt P, Freundt E, Welsh S, et al. Regulation of an ATG7beclin 1 program of autophagic cell death by caspase-8. Science. 2004;304(5676):1500-2.

[6] Tsujimoto Y, Shimizu S. Another way to die: autophagic programmed cell death. Cell death and differentiation. 2005;12 Suppl 2:1528-34. 
[7] Kaur J, Debnath J. Autophagy at the crossroads of catabolism and anabolism. Nature reviews Molecular cell biology. 2015;16(8):461-72.

[8] Choi Y, Bowman JW, Jung JU. Autophagy during viral infection - a doubleedged sword. Nature Reviews Microbiology. 2018;16(6):341-54.

[9] Heaton NS, Randall G. Dengue Virus and Autophagy. Viruses. 2011;3(8):1332.

[10] Liang Q, Luo Z, Zeng J, Chen W, Foo S-S, Lee S-A, et al. Zika Virus NS4A and NS4B Proteins Deregulate Akt-mTOR Signaling in Human Fetal Neural Stem Cells to Inhibit Neurogenesis and Induce Autophagy. Cell Stem Cell. 19(5):66371.

[11] Cao B, Parnell LA, Diamond MS, Mysorekar IU. Inhibition of autophagy limits vertical transmission of Zika virus in pregnant mice. The Journal of Experimental Medicine. 2017;214(8):2303-13.

[12] El-Hage N, Rodriguez M, Dever SM, Masvekar RR, Gewirtz DA, Shacka JJ. HIV-1 and Morphine Regulation of Autophagy in Microglia: Limited Interactions in the Context of HIV-1 Infection and Opioid Abuse. 2015.

[13] Rodriguez M, Lapierre J, Ojha C, Estrada-Bueno H, Dever S, Gewirtz D, et al. Importance of Autophagy in Mediating Human Immunodeficiency Virus (HIV) and Morphine-Induced Metabolic Dysfunction and Inflammation in Human Astrocytes. Viruses. 2017;9(8):201.

[14] Jheng JR, Ho JY, Horng JT. ER stress, autophagy, and RNA viruses. Front Microbiol. 2014;5:388.

[15] Blázquez A-B, Escribano-Romero E, Merino-Ramos T, Saiz J-C, Martín-Acebes MA. Stress responses in flavivirus-infected cells: activation of unfolded protein response and autophagy. Frontiers in Microbiology. 2014;5:266.

[16] Liang Q, Luo Z, Zeng J, Chen W, Foo S-S, Lee S-A, et al. Zika Virus NS4A and NS4B Proteins Deregulate Akt-mTOR Signaling in Human Fetal Neural Stem Cells to Inhibit Neurogenesis and Induce Autophagy. Cell stem cell. 2016;0:144554.

[17] Hamel R, Dejarnac O, Wichit S, Ekchariyawat P, Neyret A, Luplertlop N, et al. Biology of Zika Virus Infection in Human Skin Cells. Journal of virology. 2015;89:8880-96.

[18] Mizushima N, nmizu.phy2@tmd.ac.jp, Department of Physiology and Cell Biology TMaDU, Tokyo 113-8519, Japan, Yoshimori T, Department of Cellular 
Regulation RIfMD, Osaka University, Osaka 565-0871, Japan, Levine B, et al. Methods in Mammalian Autophagy Research. Cell. 2010;140(3):313-26.

[19] Dever SM, Rodriguez M, Lapierre J, Costin BN, El-Hage N. Differing roles of autophagy in HIV-associated neurocognitive impairment and encephalitis with implications for morphine co-exposure. Frontiers in Microbiology. 2015;6:1-15.

[20] El-Hage N, Rodriguez M, Dever SM, Masvekar RR, Gewirtz DA, Shacka JJ. HIV-1 and morphine regulation of autophagy in microglia: limited interactions in the context of HIV-1 infection and opioid abuse. Journal of virology. 2015;89:1024-35.

[21] Rodriguez M, Lapierre J, Ojha CR, Estrada-Bueno H, Dever SM, Gewirtz DA, et al. Importance of Autophagy in Mediating Human Immunodeficiency Virus (HIV) and Morphine-Induced Metabolic Dysfunction and Inflammation in Human Astrocytes. Viruses. 2017;9(8).

[22] Delgado MA, Elmaoued RA, Davis AS, Kyei G, Deretic V. Toll-like receptors control autophagy. EMBO J. 2008;27(7):1110-21.

[23] Liu WJ, Ye L, Huang WF, Guo LJ, Xu ZG, Wu HL, et al. p62 links the autophagy pathway and the ubiqutin-proteasome system upon ubiquitinated protein degradation. Cell Mol Biol Lett. 2016;21.

[24] Chiramel AI, Brady NR, Bartenschlager R. Divergent Roles of Autophagy in Virus Infection. Cells. 2013;2(1):83-104.

[25] Zhang Z-W, Li Z-L, Yuan S. The Role of Secretory Autophagy in Zika Virus Transfer through the Placental Barrier. Frontiers in Cellular and Infection Microbiology. 2016;6:206.

[26] Chiramel AI, Best SM. Role of autophagy in Zika virus infection and pathogenesis. Virus Research. 2017.

[27] Daffis S, Samuel MA, Suthar MS, Jr. MG, Diamond MS. Toll-Like Receptor 3 Has a Protective Role against West Nile Virus Infection. Journal of Virology. 2008.

[28] Tabeta K, Georgel P, Janssen E, Du X, Hoebe K, Crozat K, et al. Toll-like receptors 9 and 3 as essential components of innate immune defense against mouse cytomegalovirus infection. Proceedings of the National Academy of Sciences. 2004.

[29] Shi C-S, Kehrl JH. MyD88 and Trif Target Beclin 1 to Trigger Autophagy in Macrophages. Journal of Biological Chemistry. 2008;283:33175-82. 


\section{CHAPTER 6. INFECTION WITH ZIKV CAUSES GROWTH AND BRAIN}

\section{ABNORMALITES IN THE PUPS OF AUTOPHAGY-DEFICIENT MICE}

\subsection{Introduction}

ZIKV is a neurotrophic flavivirus associated with microcephaly and other fetal neurological disorders collectively known as Congenital Zika Syndrome [1-3]. Although most human infections of ZIKV are typically transmitted by the Aedes mosquito, some virus can also spread through sexual contact, blood transfusion [4-7] and through vertical transmission from infected pregnant mothers to their fetuses [8-10]. Similar to the infection caused by other arboviruses such as DENV or CHIKV, ZIKV-infected individuals typically develop mild symptoms. Unlike other arboviruses, in-utero exposure with ZIKV can cause congenital malformations in the fetus such as microcephaly [11, 12], and other overt congenital abnormalities including fetal death, placental insufficiency, fetal growth restriction and central nervous system injury [13].

Microcephaly is a neurodevelopmental disorder in newborns, characterized by an abnormal smaller sized head when compared to babies of the same sex and age. The significant reduction in brain size accompanied by intellectual disability are believed to be caused by impaired cell proliferation and the death of cortical progenitor cells and their neuronal progeny [14]. Genetically, microcephaly is linked to the alterations in genetic loci microcephalin (MCPH). Twelve of the MCPH loci (MCPH1-MCPH12) including microcephalin-1 (MCPH1), the WD repeat-containing protein 62 (WDR62), the CD5 regulatory subunit associated protein 2 (CDK5RAP2), the cancer susceptibility candidate 5 (CASC5), the abnormal spindle-like primary microcephaly (ASPM) have been already mapped [15]. Many of these genes encode proteins localized at the centrosome or with 
centrosome-related activities and play an important role in cell cycle progression, cell division and formation of the mitotic spindle [16]. More importantly, significant downregulation of several of the microcephaly-associated genes was detected in ZIKVrelated studies [17-19], suggesting a direct mechanistic link of ZIKV infection to microcephaly at the molecular level.

Despite the outpouring literature related to in utero transmission of $\mathrm{ZIKV}$, little work has been done to elucidate mechanism associated with ZIKV-induced neurological deficits during gestation. Furthermore, how viral particles and other causal factors transmigrate across the placental barrier and the blood-brain barrier, inflicting neuropathology in the fetus, is still unclear. Recently we have shown that ZIKV can modulate the autophagy pathway in glial (astrocytes and microglia) cells while silencing of the autophagy gene, beclin1 leads to increased inflammation in ZIKV-infected glia [2022]. Beclin1 is a component of the phosphatidylinositol 3-kinase nucleation complex which regulates the initiation stages of the autophagy pathway [23]. Autophagy is a multi-step intracellular degradative system which delivers cytoplasmic contents including misfolded proteins, dysfunctional organelles and components of pathogens to lysosomes. The process is characterized by the formation of the autophagosome, a unique double-membrane structure, which is degraded upon fusion with lysosome [23]. A malfunction in this pathway has been implicated in a number of neurological diseases such as Alzheimer's and Parkinson's diseases as well as in human immunodeficiency virus (HIV)-associated neurological disorders [24].

The goal of the studies proposed in Aim 3 was to explore the role of autophagy pathway, specifically the protein Beclin1 in the mechanism associated with ZIKV-induced 
abnormal fetal development during gestation using ZIKV-infected timed-pregnant wildtype C57BL/6J and Beclin1 deficient $\left(\operatorname{Atg}^{+/-}\right)$mice. We report for the first time that three different phylogenetic strains (Honduras-R103451, Puerto Rico-PRVABC59 and UgandaMR766) of ZIKV infect timed-pregnant Beclin1 $\left(A \operatorname{tg} 6^{+-}\right)$deficient and wild-type (C57BL/6J) animals, while only pups born to ZIKV-R103451-infected dams exhibit growth impairment, specifically those born to viral-infected $\operatorname{Atg} 6^{+/-}$dams. Our data indicate that significant downregulations of the microcephaly related genes MCPH1, ASPM, WDR62, and CASC5 in the brains and significant increases in pro-inflammatory cytokines secreted by the glial cells are associated with impaired growth and brain development of the pups born to ZIKV-infected autophagy deficient mice. We detected ZIKV NS1 and Env protein in pup's brain in the absence of ZIKV genome.

\subsection{Materials and Methods}

\subsubsection{Ethics statement}

Animal work was conducted in accordance with the guidelines of the National Institutes of Health (NIH) guide for the care and use of laboratory animals. Animal experiments and associated protocols were reviewed and approved by the Florida International University institutional animal care and use committee (IACUC).

\subsubsection{Animal model and timed pregnancy}

C57BL/6J (stock \# 000664) and B6.129X1-Becn1tm1Blev/J (stock \# 018429) mice on a C57BL/6J background were procured from The Jackson Laboratory (Bar Harbor, ME, USA) and bred in the animal facility at Florida International University. For the timedpregnancy studies, male and female mice aged 8 to 15 weeks were kept isolated for at least 24 hours before mating. After 24 hours, females were introduced into the breeding cage 
with the male in the early evening and monitored periodically for 48 hours after initial introduction to detecting for the presence of a vaginal plug as an indication of mating. The day on which the plug is observed is considered day zero (E0) and mouse weight was recorded every three days to ensure the pregnancy. Pregnant dams received anti-IFNAR1 mouse monoclonal antibody (MAR-5A3, Leinco Technologies) at $2 \mathrm{mg} / \mathrm{Kg}$ body weight via intraperitoneal (IP) route at gestational date of E8 followed by subcutaneous (SC) injection with $10^{3}$ Plaque Forming Unit (PFU) of ZIKV (R103451) in 50 $\mu$ L of PBS or PBS only (mock) at gestational date of E9. A booster dose of anti-IFNAR1 at $0.5 \mathrm{mg} / \mathrm{Kg}$ dose was administered by IP at 2 and 4 days' post-infection. In one set of experiment, pregnant dams $(\mathrm{N}=8)$ were sacrificed, and maternal placenta and organs including the liver, spleen, heart, and brain from both mother and fetus were collected post-mortem on gestation day E17. In the other set of experiment, pregnant dams $(\mathrm{N}=8)$ were allowed to deliver their pups, which were monitored through 6 weeks of age for any signs of ZIKV infection including evaluation of postnatal growth. Tissues (brain, kidney, and liver) harvested postmortem were snap frozen for molecular and histopathological analysis (Figure 37). 


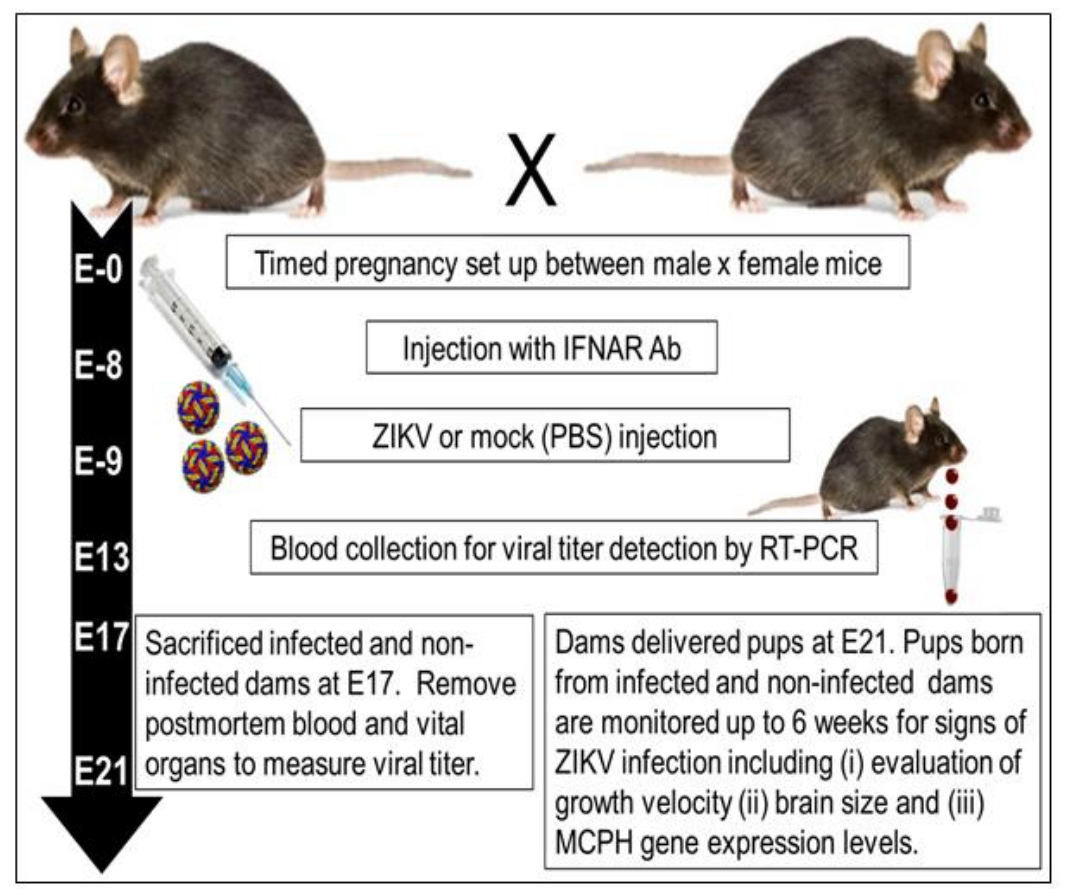

Figure 37. Experimental design for mouse infection model.

Timed pregnancy was set up and monitored for the duration of gestation. On embryonic day 9 (E9) animals were treated with IFNAR antibody $(2 \mathrm{mg} / \mathrm{kg}$, IP). On E10 animals were challenged with ZIKV by subcutaneous route $\left(10^{3} \mathrm{PFU} / 50 \mu \mathrm{L}\right)$ on foot pad. Mock animals received 50 $\mu$ L PBS. On E11 and E13, booster doses of IFNAR antibody were administered to animals $(0.5 \mathrm{mg} / \mathrm{kg}$, IP). Blood was collected on E13 for ZIKV titer detection. On E17, dams were sacrificed for collection of the fetus and vital organs including placenta, while another set of experiments, pregnant dams were allowed to deliver their pups which were monitored for potential growth impairments. Pregnant dams ( $\mathrm{N}=10 /$ strain) delivered $(\mathrm{N}=5-$ 8) pups.

\subsubsection{Real-Time PCR}

Viral RNA from serum and tissues collected at various time-points from both ZIKV-infected and uninfected animals were extracted using QIAamp Viral RNA mini kit (Qiagen, Valencia, CA, USA). Cellular RNA was extracted by RNeasy Mini Kit (Qiagen, Valencia, CA, USA). Viral RNA was amplified using iTaq universal SYBR Green onestep PCR kit (Bio-Rad, USA) and $10 \mu \mathrm{M}$ of the ZIKV primers (Sigma-Aldrich, USA). A standard curve was prepared from a 10-fold dilution of previously quantified ZIKV stock solution with known titer, and viral titer was expressed as RNA copies equivalent of $\log _{10}$ 
PFU/mL. MCPH1, ASPM, CASC5, and WDR62 gene expressions were measured from total RNA extracted from fetal and pup brain using RT-PCR.

$\underline{\text { Primer sequences }}$

ZIKV: Forward: 5'-CCGCTGCCCAACACAAG-3'

Reverse: 5'-CCACTAACGTTCTTTTGCAGACAT-3'

MCPH1: Forward 5'- AAGAAGAAAAGCCAACGAGAACA-3'

Reverse 5'-CTCGGGTGCGAATGAAAAGC-3',

ASPM: Forward 5'-CCGTACAGCTTGCTCCTTGT-3'

Reverse 5'-GGCGTTGTCCAATATCTTTCCA-3',

CASC5: Forward 5'-TCGCTGAAGTGGAAACAGAAAC-3'

Reverse 5'-TATCTGAGCAAGGGTCTCTGCG-3'

WDR62: Forward 5'-GCTGACAAATGGCAAGCTG-3'

Reverse 5'-GATGGTCTTGAGGGGTTCCT-3'.

\subsubsection{Labeling with Hematoxylin \& Eosin}

Brain tissues from ZIKV-infected and non-infected pregnant dams and their offsprings were each embedded using optimal cutting temperature (OCT) compound. Cryostat sectioned slices of 5-micron thickness were stained with hematoxylin and eosin (H\&E) as described previously by us [25]. Briefly, tissues were exposed to xylene and rehydrated with absolute ethanol, 95 and $70 \%$ ethanol, followed by staining with hematoxylin dye for $15 \mathrm{~min}$, before washing with distilled water. Tissues were stained with eosin for 20 secs, dehydrated with gradient ethanol after washing with tap water, and tissues were then cleared by xylene and mounted using mounting media for visualization. 
Images were acquired using a Zeiss (Germany) inverted fluorescence microscope with a 560 Axiovision camera using 20, 40, or 63X objectives.

\subsubsection{Murine mixed glial cell culture}

For primary murine glial culture, post-natal day 4-6 (P4-P6) ATG6 ${ }^{+/-}$mice and C57BL/6 littermates were separated according to phenotypic coat color and sacrificed according to IACUC guidelines as described previously by us [26]. Growth medium contained Dulbecco's Modified Eagle's Medium (DMEM; Invitrogen, Carlsbad, CA, USA) supplemented with glucose (2 mg/mL; Sigma-Aldrich), $\mathrm{Na}_{2} \mathrm{HCO}_{3}$ (6 mM; Invitrogen), $10 \% \mathrm{v} / \mathrm{v}$ heat-inactivated fetal bovine serum (FBS; Hyclone, Logan, UT, USA), and $1 \%$ penicillin/streptomycin (100 U/mL / $100 \mu \mathrm{g} / \mathrm{mL}$; Invitrogen) [27]. Striata were isolated and dissociated mechanically and enzymatically with $0.25 \%$ trypsin containing DNase $(2.5 \mathrm{mg} / \mathrm{ml})$ centrifuged, triturated, and twice filtered through 40 -micron nylon mesh cell strainer. Cells were then plated and maintained in plates coated with PolyL-lysine $(0.1 \mathrm{mg} / \mathrm{ml})$. Cultures were maintained for $5-10$ days at $37^{\circ} \mathrm{C}$ and $5 \% \mathrm{CO}_{2}$.

\subsubsection{Statistical analysis}

Results are reported as mean $\pm \mathrm{SEM}$ of three independent experiments. Data were analyzed using analysis of variance (ANOVA) techniques followed by appropriate post hoc test for multiple comparisons (GraphPad Software, Inc., La Jolla, CA, USA). An alpha level (p-value) of $<0.05$ was considered significant. 


\subsection{Results}

\subsubsection{Timed-pregnant transiently immunosuppressed $\mathrm{C57BL} / 6 \mathrm{~J}$ and $\mathrm{Atg}^{+/-}$mice $^{-}$ were susceptible to ZIKV infection}

We explored the role of the autophagy pathway in ZIKV infection using timedpregnant Beclin1-deficint (ATG6 ${ }^{+/}$) and wild type (C57BL/6J) mice model. A schematic diagram of the experimental design with various end-point is shown in Figure 37 and is described in the material and methods section. IFNAR1 blocked pregnant dams were infected with ZIKV (R103451) at a dose of $10^{3}$ PFU in 50 $\mu$ L of PBS. We selected the viral strain R103451 based on the higher infectivity of the strain to mice compared to other strains (MR766 and PRVABC59) and its close relation with the Brazilian strain. The body weights of each pregnant mouse were measured before the detection of a vaginal plug and throughout the gestation period in order to confirm the pregnancy and weight gain patterns in pregnant dams (Figure 38A). Interestingly, infection of beclin1 deficient $\left(\operatorname{Atg} 6^{+/-}\right)$dams with all three strains of ZIKV showed relatively less gain in body weight compared to wild type (C57BL/6J) dams, although both strains delivered an equal amount of offspring $(\mathrm{N}=$ 5 - 8) per litter. It is important to point out that the size of a litter delivered by the $\operatorname{Atg} 6^{+/-}$ dams (crossed with an Atg6 $6^{+-}$sire) is controlled genetically. Approximately, $50 \%$ of the pups delivered will be heterozygous for the beclin 1 gene $\left(\operatorname{Atg} 6^{+-}\right)$with a brown coat, $25 \%$ will be homozygous for the Beclin1 gene $\operatorname{Atg} 6^{+/+}$with a black coat, while $25 \%$ will not express the beclin 1 gene $\left(\operatorname{Atg} 6^{-/}\right)$and die in utero. Next, we monitored the survival rates

of infected dams for up to three weeks. ZIKV-infected $\operatorname{Atg} 6^{+/}$dams showed significantly lower survival rate compared to mock-infected Atg6 $6^{+/-}$as well as ZIKV-infected C57BL/6J 
dams (Figure 38B). Likewise, the increased fatality was observed in animals infected with the other viral strains, specifically after infection with ZIKV-MR766 (Figure 39A).

Viral infection measured in serum collected on E13 or 4 dpi by RT-PCR showed viremia levels in the range of $10^{3} \mathrm{PFU} / \mathrm{mL}$ in both wild type and $\operatorname{Atg}^{+/-}$dams with no significant difference between strains (Figure 38C). Analysis of viral titers in various organs removed post-mortem on E17 showed levels of viral RNA in the following order: placenta $>$ spleen $>$ liver $>$ heart with the lowest titer detected in the brain, irrespective of mice strains, indicating that ZIKV infection or replication in infected mice is not dependent on Beclin1 (Figure 38D). We did not detect measurable ZIKV titer in the brain nor any other vital organs collected post-mortem from pups born to ZIKV-infected C57BL/6J and $\operatorname{Atg} 6^{+/-}$dams.
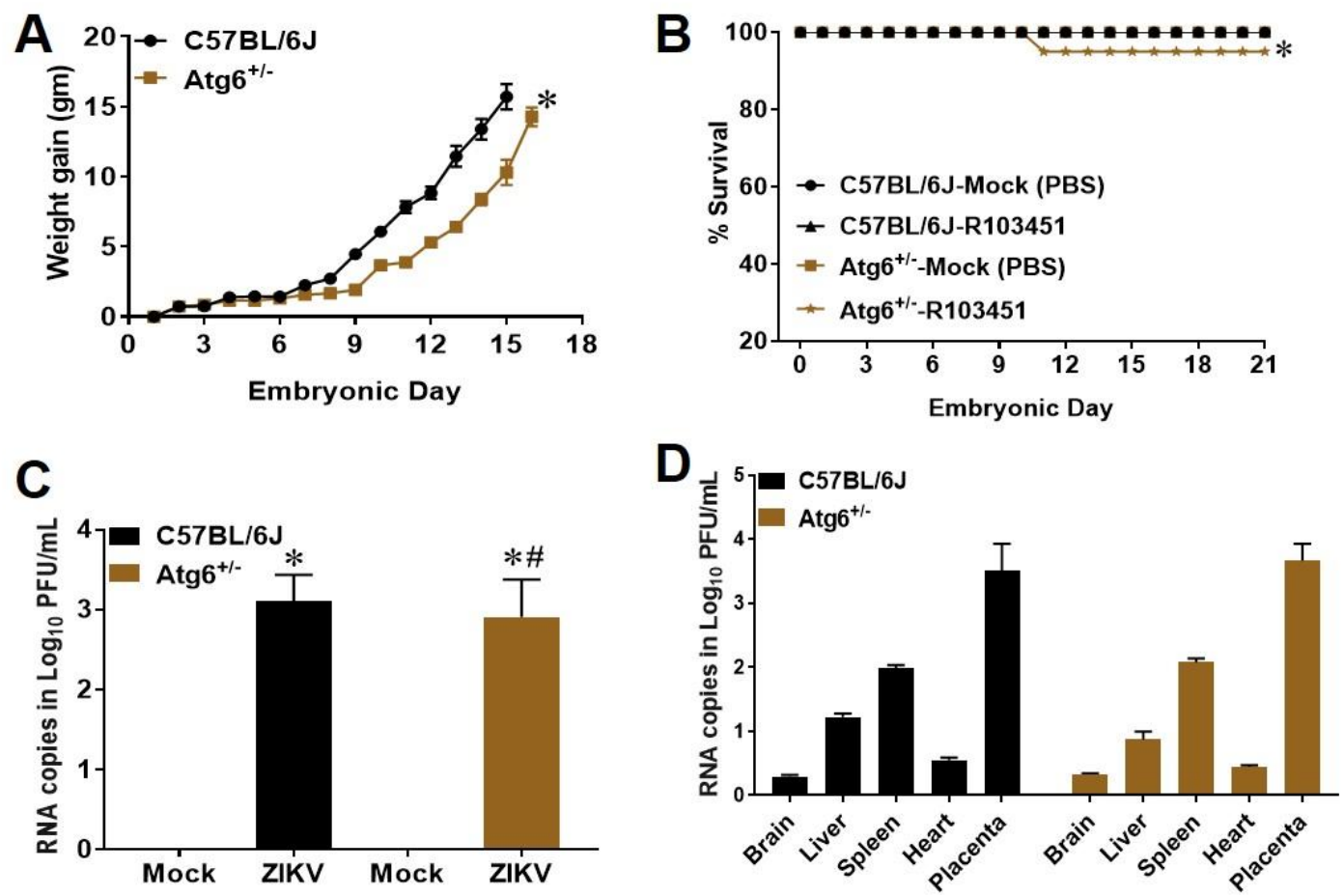

Figure 38. ZIKV establish productive infection in numerous organs of wild type and Beclin1 deficient pregnant mice. 
(A) Body weight gain of wild type C57BL/6 and Atg6 $6^{+/-}$pregnant dams after detection of vaginal plug. (B) Survival curve of C57BL/6 and Atg6 ${ }^{+/-}$pregnant dams after infection with ZIKV (R103451) compared with respective mock infected pregnant dams. (C) ZIKV titers in the serum from infected dams as measured by RT-PCR on E13 and expressed as RNA copies equivalent to $\log _{10} \mathrm{PFU} / \mathrm{mL}$. Numbers are based on a standard curve prepared from known viral stocks. (D) ZIKV titers measured by RT-PCR using RNA extracted from different organs removed post-mortem from infected dams sacrificed on E17. Data are presented as mean \pm SEM from 3 independent experiments and analyzed using one-way ANOVA with Bonferroni post hoc correction. *p $<0.05 \mathrm{Vs}$ control.

Infection with phylogenetically different strains of ZIKV (PRVABC59 and MR766) also showed similar viral titers in serum and organs removed postmortem (Figure 39B and C). However, infection with ZIKV- PRVABC59 showed significantly lower viral titer in serum of $\mathrm{Atg}^{+/-}$dams compared to their wild-type counterparts (Figure 39B). Since we detected high mortality among the dams infected with ZIKV-MR766, we sought to confirm that the observed mortality was caused by ZIKV infection using IFNAR1 knock-out mice infected with increasing doses $\left(10^{1}, 10^{2}, 10^{3}\right.$ and $\left.10^{4} \mathrm{PFU} / \mathrm{ml}\right)$ of ZIKV-MR766. We detected an infection-dose dependent decrease in survival rate of the mice (Figure 39D). 

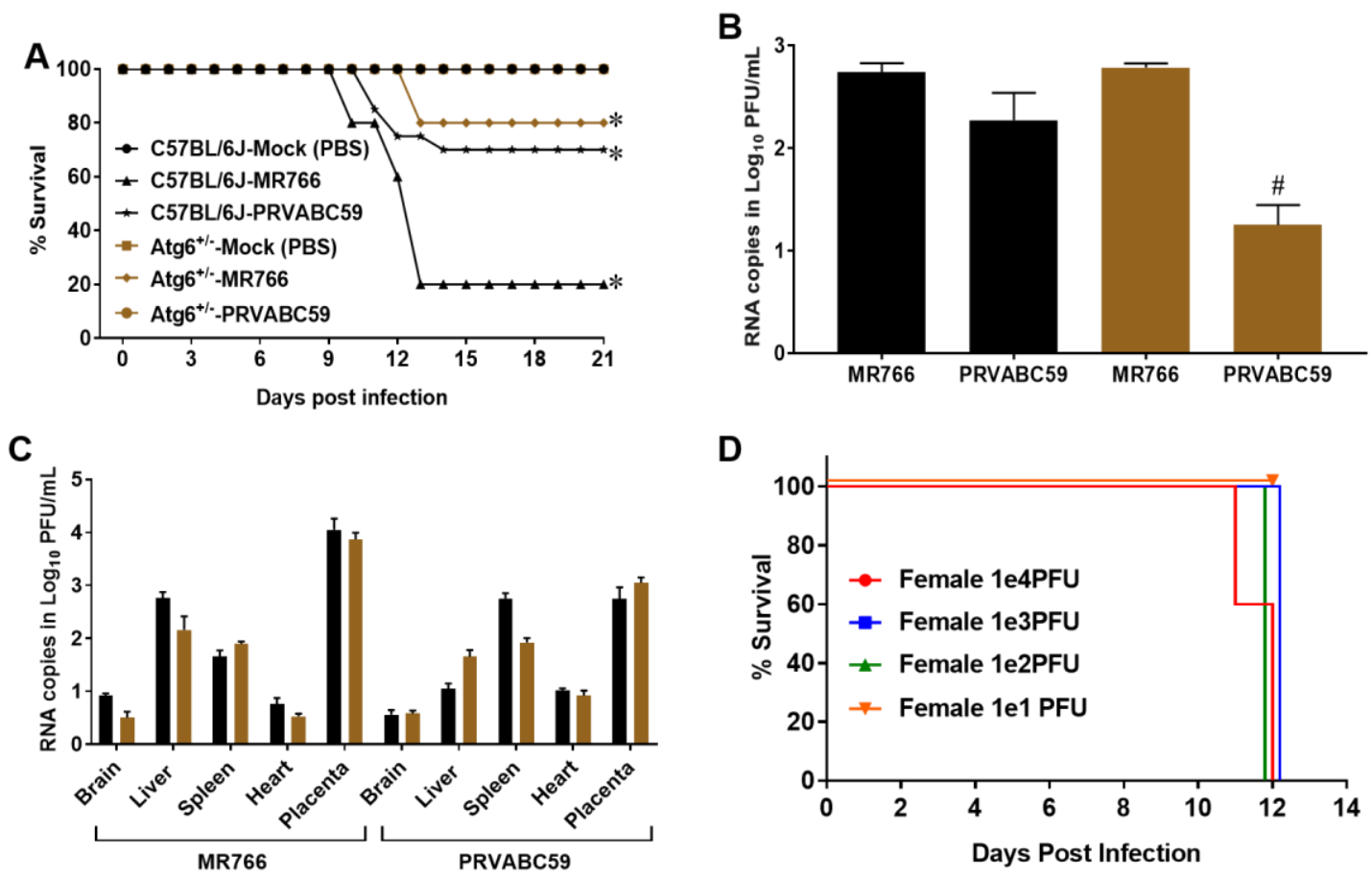

Figure 39. Infection with Different strains of ZIKV (MR766 and PRVABC59).

Percent survival of C57BL/6 and Atg6 ${ }^{+/-}$pregnant dams (A) after infection with ZIKV (MR766 and PRVABC59) compared with mock infected dams. (B) Viral RNA isolated from serum was used to analyze viral titer by RT-PCR. (C) Cellular RNA extracted from organs removed post-mortem was used to analyze viral titer by RT-PCR. C57BL/6 (black bars) and $A \operatorname{tg} 6^{+-}$(brown bars). (D) Percent survival from IFNAR ${ }^{-/}$knockout mice infected with MR766 (10 $10^{1}$ to $\left.10^{4} \mathrm{PFU} / \mathrm{mL}\right)$ shows $100 \%$ mortality on Day-12 post infection. $\mathrm{N}=8$ pregnant dams per group. Error bars show mean \pm SEM for 3 independent experiments. ${ }^{*} \mathrm{p}<0.05$ vs. respective mock infected pregnant dams and ${ }^{\#} \mathrm{p}<0.05$ vs. C57BL/6J (Two-way ANOVA followed by Tukey's test).

\subsubsection{Beclin1 deficiency impairs survival and growth in offspring exposed to ZIKV} in utero

The major concern related to ZIKV is its association with neurodevelopmental disorders and growth retardation in newborns and infants. Impaired autophagic pathway in the brain is often associated with neurodegenerative disorders including viral neuropathogenesis [24]. However, whether the autophagy pathway enhances or protects ZIKV-mediated impairment in fetal growth is still not explored. To this end, pups born from mock and ZIKV-infected C57BL/6J and Atg6 ${ }^{+/-}$dams were monitored for mortality 
and adverse morphological abnormalities by measuring body weight and length, for up to 21 days after birth. A slight decrease in the survival rate of pups born to ZIKV-infected C57BL/6J and Atg6 ${ }^{+-}$dams was noted (Figure 40A). Pups born to ZIKV-PRVABC59 and ZIKV-MR766-infected dams did not show significant differences in survival rate (Data not shown).

Next, we monitored the survived pups born from ZIKV and mock-infected dams until three weeks of birth. Growth development, as measured by the body weight and length, was similar among survived pups born to mock and ZIKV-infected C57BL/6J dams (Figure 40B). Morphological abnormalities as indicated by lower body weight and length was detected after 14 days post birth in the pups born to $A \operatorname{tg} 6^{+/-}$dams infected with ZIKV$\mathrm{H}$ (Figure 40C and D). The visible difference in body size was detected in 1 of every 3.54 (25-30\%) pups (within the same litter) born to ZIKV-H-infected Atg6 ${ }^{+/-}$dams. Pups born with growth and brain impairment were brown coated and heterozygous for the beclin1 gene $\left(\mathrm{Atg}^{+/-}\right)$(Figure 40C). The average body weight in these pups was around $6.8 \mathrm{gm}$ as compared to $8.0 \mathrm{gm}$ which is a typical weight of a 3 week old mouse. The average body length of the lean pups was also significantly smaller measuring at around $5.30 \mathrm{~cm}$ as compared to $6.01 \mathrm{~cm}$ which is a typical weight of a 3 week old mouse (Figure 40D). It is important to note that no significant growth abnormalities were measured in pups born to mock infected dams or dams infected with the ZIKV-PRVABC59 and the ZIKV-MR766, irrespective of murine strains (data not shown). After 21 days, both small and typical sized pups were sacrificed and brains were removed for further analysis. As expected, brain removed from the smaller sized pups were significantly smaller in size and grossly underdeveloped almost liquid in consistency, while the brains from the typical sized pups 
had a well-defined brain and solid in consistency (Figure 40C). Overall, the data show that attenuation of autophagy, and the protein Beclin1 leads to growth and brain impairment in pups exposed in utero to the ZIKV (R103451).

A
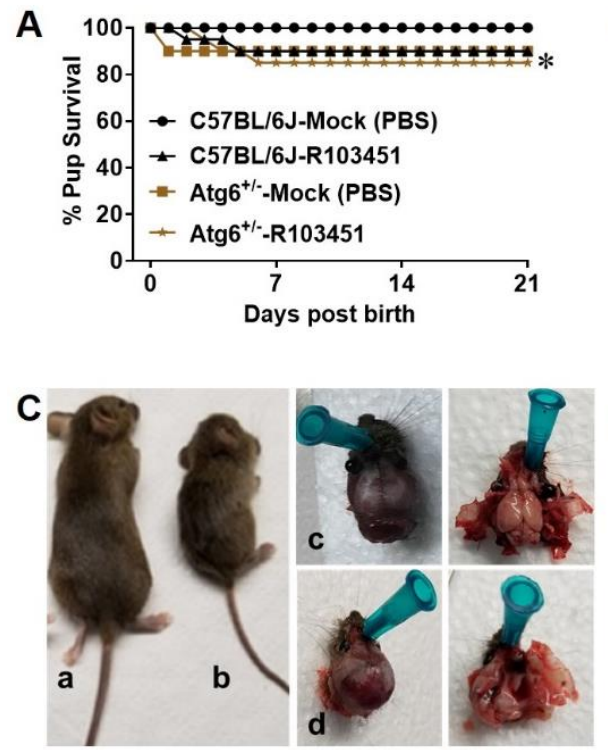
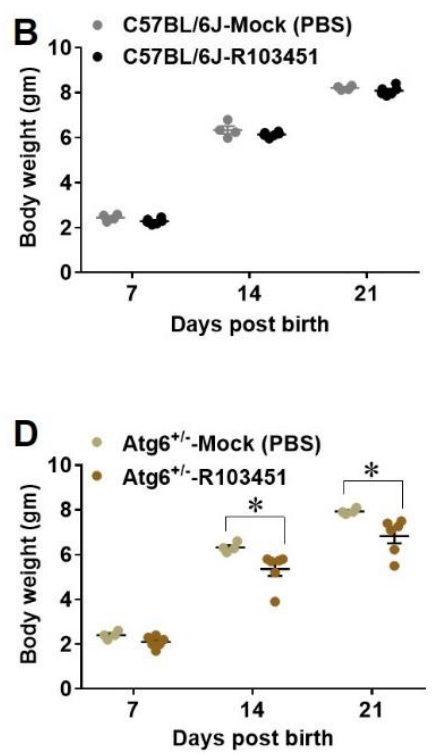
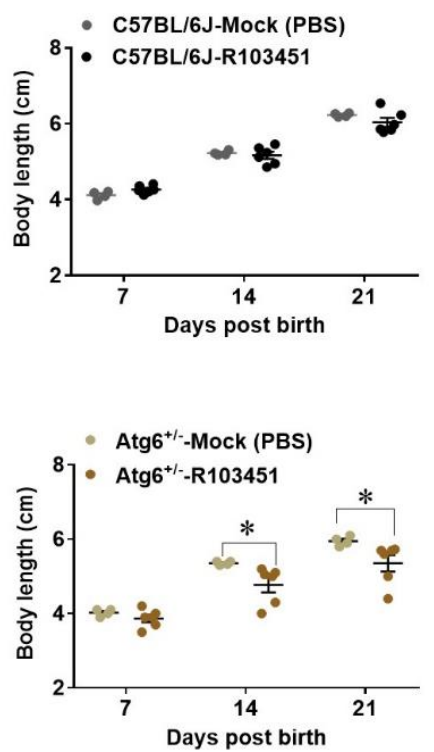

Figure 40. Beclin1 deficiency impairs survival and growth in offspring exposed to ZIKV in utero.

A) Survival curve of pups born to ZIKV-infected and mock-infected wild types and /or Atg6 $6^{+-}$dams. (B) Body weight and length of pups born from wild type dams measured at day 7, 14 and 21. (C) Representative images of pups born to $A \operatorname{tg} 6^{+/-}$dams exposed to PBS (1) or ZIKV (2) in utero showing significant delay in growth and brain development. (D) Body weight and length of pups born from $\operatorname{Atg}^{+/-}$dams measured at day 7, 14 and 21 . Data are presented as mean \pm SEM from 5 independent experiments and analyzed using two-way ANOVA with Bonferroni post hoc correction. ${ }^{*} \mathrm{p}<0.05$ Vs respective mock control.

\subsubsection{Expression of microcephalic genes was decreased in offspring exposed to ZIKV}

\section{in utero}

Exploring potential causal factors associated with the growth and brain impairment detected in pups born to ZIKV-H-infected $\mathrm{Atg}^{+/-}$dams, we first measured viral titers in various organs from pups removed post-mortem. Although viral RNA was not detected (data not shown), the non-structural (NS) protein-1 and structural protein (Env) were 
detected in postmortem brain of the pups exposed to ZIKV in utero (Figure 41A). Microscopic appearance of the frontal cortex stained with H\&E showed no visible sign of aberrant morphology of neurons in mock-infected brain tissues removed post-mortem (Figure 41B_top panel). In contrast, ZIKV-infected brain tissues showed signs of necrotic neurons with shrunken neuronal cell bodies (Figure 41B_bottom panel).
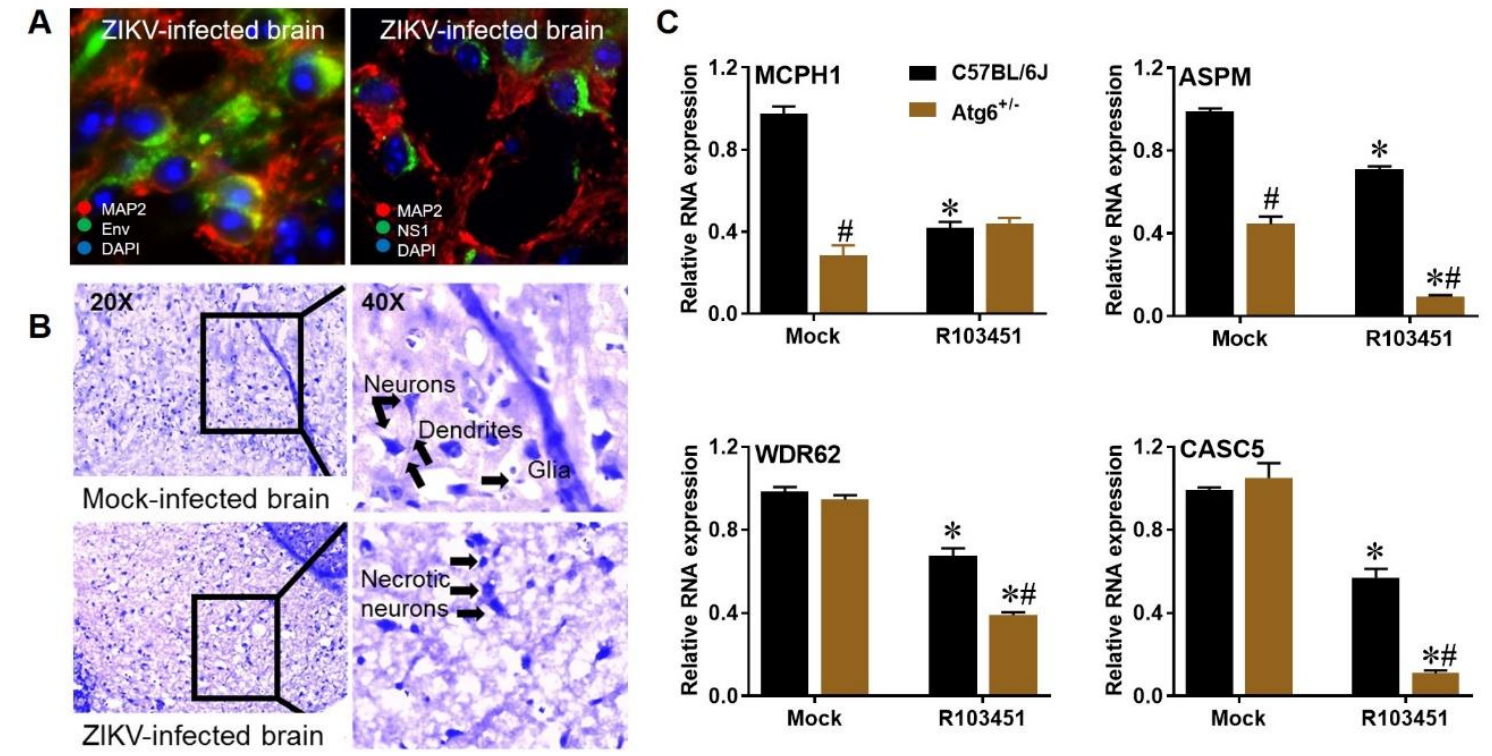

Figure 41. Expression of microcephalic genes was decreased in offspring exposed to ZIKV in utero.

(A) Representative images of brain tissues labeled by immunofluorescence. Red color represents the microtubule-associated protein 2 (MAP-2) indicator of mature neurons, the green color represents ZIKV Structural protein (Env) and Non-structural protein-1 (NS-1), and the blue color indicates DAPI-labeled nuclei. (B) Hematoxylin \& Eosin (H\&E) staining of brains removed post-mortem exposed to PBS (top panel) and ZIKV (bottom panel) in utero. Representative images were acquired at 20 and $40 \mathrm{X}$ magnification. (C) RNA expression of the microcephaly associated genes (MCPH1, ASPM, WDR62, and CASC5) were measured by RT-PCR. C57BL/6 (black bar) and Atg6 ${ }^{+/-}$(brown bar). Error bars show mean \pm SEM for 3 independent experiments. The data were analyzed by Twoway ANOVA followed by Tukey's multiple comparison test. ${ }^{*} \mathrm{p}<0.05$ vs. respective mock infected strain, ${ }^{\mathrm{p}}<0.05$ vs. C57BL/6J.

Expression levels of microcephaly $(\mathrm{MCPH})$ related genes previously linked to stillbirth, brain development, and microcephaly in ZIKV-exposed fetus [33, 34] were 
measured in post-mortem brain tissues of pups exposed to ZIKV infection in utero. A decrease in RNA expression levels of about 2.5-fold in MCPH1 and ASPM and about 1.7fold in CASC5 were detected in post-mortem brain tissues of pups exposed to in utero ZIKV infection when compared to post-mortem brain tissues exposed to PBS (Figure 1Figure 41C). Interestingly, expressions of the MCPH related genes in brain tissues removed post-mortem from pups born to $\mathrm{ZIKV}$-infected $\mathrm{Atg} 6^{+/-}$dams were further reduced by about 3-fold compared to pups born from ZIKV-infected C57BL/6J dams (Figure 41C).

Overall, the data showed significant impairment in the expression of the microcephalic related genes, which may be associated with the increased mortality rate and growth retardation in offspring born from ZIKV-infected Atg6 ${ }^{+-}$dams.

\subsubsection{Beclin1 deficiency enhances the secretion of inflammatory molecules in ZIKV- infected mouse mixed glial cells}

Since glial cells are the principal cell types involved in the release of neuroinflammatory molecules, they are usually considered the culprit in many viral pathology and autophagy pathway might influence the levels of inflammatory molecules secretion by infected glial cells $[35,36]$. To this end, mixed glia (astrocytes and microglia) were isolated from the whole brain of either $\mathrm{C} 57 \mathrm{BL} / 6 \mathrm{~J}$ or $\operatorname{Atg} 6^{+/-}$pups and seeded as previously described by us [26]. First, we measured infectivity of ZIKV (R103451) in the glial cells and showed that murine mixed glial cells were permissive to ZIKV infection (Figure 42A), irrespective to murine strain. Secretion of the inflammatory chemokines RANTES and MCP-1 and the cytokine IL-6 were measured in non-infected, and ZIKV (R103451)-infected (MOI of 0.1) glial culture supernatants by ELISA and showed a significant increase in inflammatory molecules at 24, 48 and 72 hpi with ZIKV infection 
(Figure 42B). Significant differences in ZIKV-induced secretion of inflammatory molecules were detected between glia derived from $\operatorname{Atg} 6^{+/-}$pups when compared to glia derived from C57BL/6J pups. After twenty-four hours, secretion of RANTES was 2.5-fold, MCP-1 was 1.4-fold, and IL-6 was 1.6-fold higher in ZIKV-infected glia derived from Atg6 $6^{+/-}$pups when compared to similar treated glia derived from C57BL/6J pups (Figure 42B). More importantly, the secretions of RANTES and MCP-1 in supernatant derived from $A \operatorname{tg} 6^{+-}$glia infected with ZIKV (R103451) remained significantly higher throughout the experiment (Figure 42B).
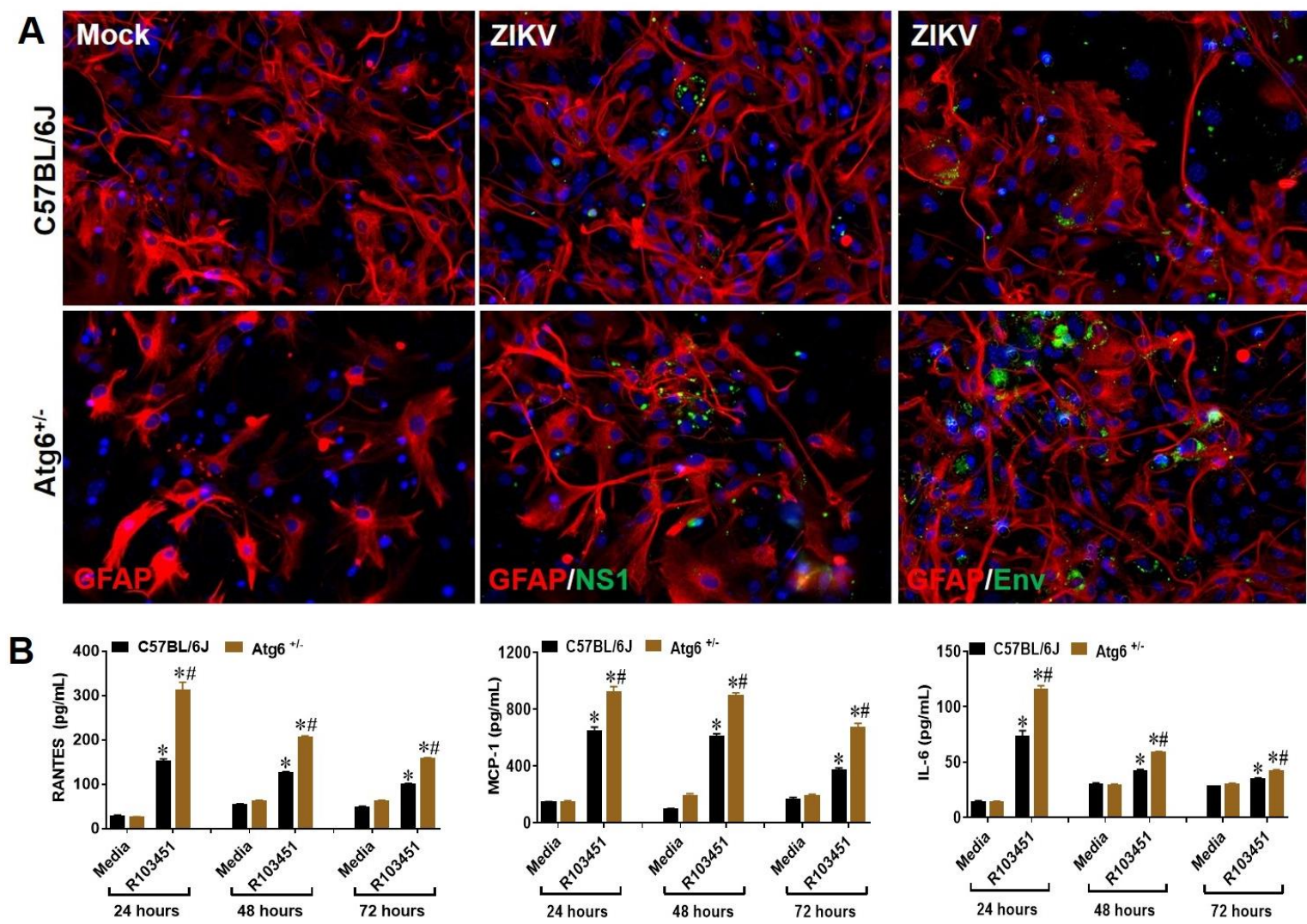

Figure 42. ZIKV infects mixed mouse glia and induces inflammatory molecules.

(A) Representative images of mouse glia from C57BL/6 and Atg6 $6^{+/-}$pups infected with ZIKV (MR766, R103451, and PRVABC59) for $24 \mathrm{~h}$ and immunofluorescently labeled with GFAP (green), ZIKV Env (red) and DAPI nucleus (blue). (B-D) Secretion of RANTES, IL6 and MCP-1 detected in glial supernatants infected with ZIKV (R103451) 
were measured after 24, 48 and 72 hpi by ELISA. Glia derived from C57BL/6 pups (black bar) and glia derived from Atg6 $6^{+/-}$pups (brown bar). Data are presented as mean \pm SEM from 3 independent experiments and analyzed using two-way ANOVA with Bonferroni post hoc correction. *p $<0.05$ Vs Control, \# $\mathrm{p}<0.05$ Vs C57BL/6J.

\subsubsection{Beclin1 deficiency enhances the secretion of inflammatory molecules in NS1, and Env protein treated mouse mixed glial cells}

Since we detected ZIKV proteins (NS1) but not the virion in brains of pup exposed to ZIKV in utero, we examined whether treatment with a nonstructural protein NS1 and a structural protein Env induce secretion of inflammatory molecules by the mixed glia. A protein concentration of 50nM NS1 and Env was selected based on serum concentration of these proteins reported in a severe flaviviral infection [37]. We found that exposure with NS1 and Env proteins significantly increased the secretion of RANTES, MCP-1, IL-6 and TNF- $\alpha$ (Figure 43) in both C57BL/6J-derived and Atg6 $6^{+/-}$-derived glia. However, similar to the ZIKV infection, both NS1 and Env proteins had more inflammatory effects on the $\operatorname{Atg} 6^{+/-}$-derived glia when compared to C57BL/6J-derived glia. Secretion of TNF- $\alpha$ was drastically increased in $\operatorname{Atg} 6^{+/-}$-derived glia after 24 and 96 hpi when compared to similar treated C57BL/6J-derived glia.

Overall, the data show an important role of the protein Beclin1 in regulating the release of inflammatory molecules in response to pathogenic insults to the host, and that attenuating autophagy leads to increased release of inflammatory molecules that could account for the maladaptive abnormalities detected in pups born from ZIKV infected $\operatorname{Atg} 6^{+/-}$dams. 

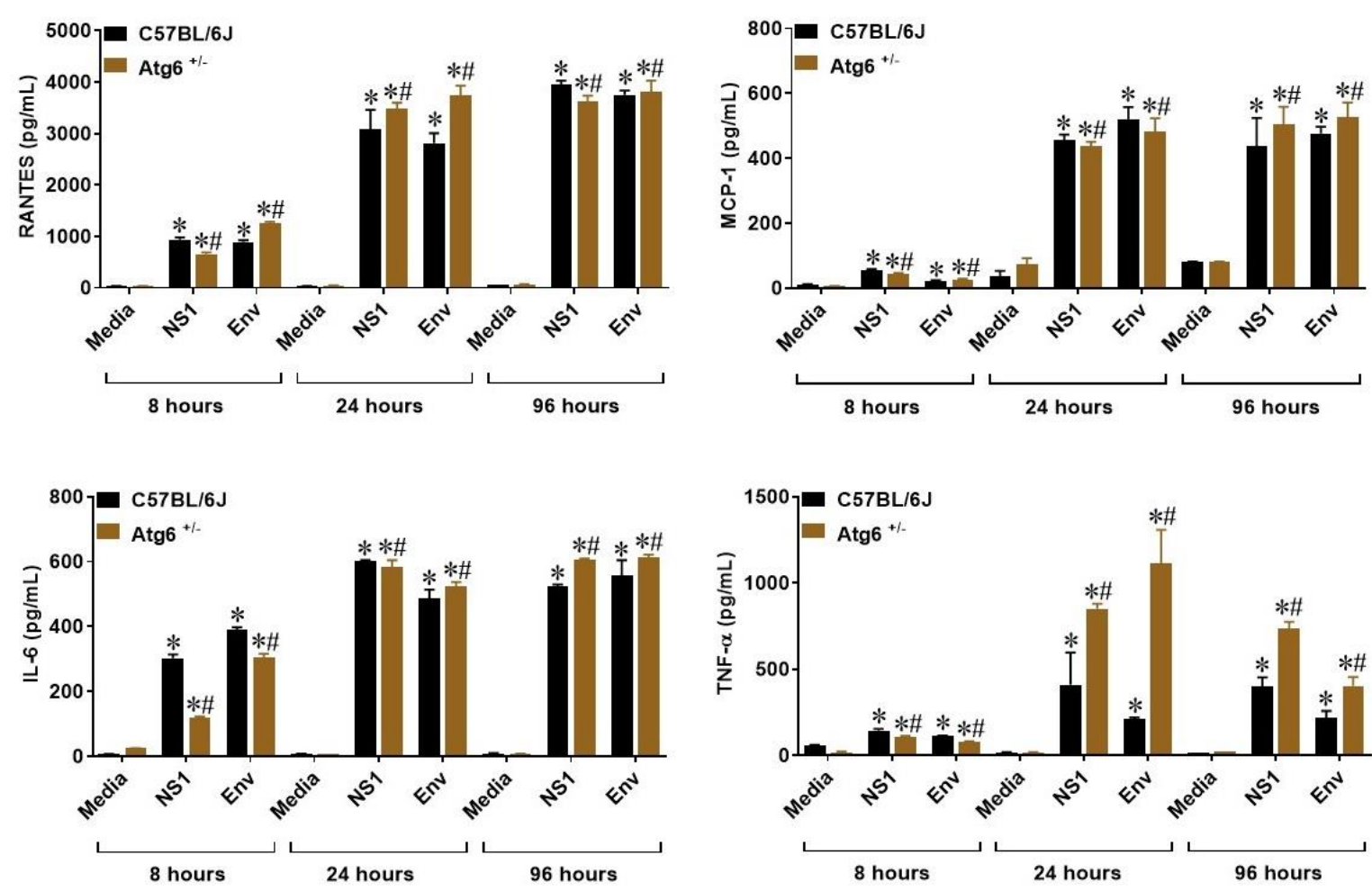

Figure 43. ZIKV NS1 and Env proteins cause increased secretion of several inflammatory molecules in $\mathrm{Atg}^{+/-}$-derived glia.

Secretion of RANTES, MCP-1, IL6 and TNF- $\alpha$ in supernatants of NS1 and Env-treated mouse glial cells was measured after 8, 24 and 96 hpi by ELISA. Glia derived from C57BL/6 pups (black bar) and glia derived from Atg6 ${ }^{+/-}$pups (brown bar). Data are presented as mean \pm SEM from 3 independent experiments and analyzed using one-way ANOVA with Bonferroni post hoc correction. *p $<0.05 \mathrm{Vs}$ Control, \#p <0.05 Vs C57BL/6J.

\subsection{Discussion}

In this study, we reported for the first time that three different phylogenetic strains of ZIKV infects timed-pregnant Beclin1 $\left(\mathrm{Atg}^{+/-}\right)$deficient and wild-type (C57BL/6J) animals, while only pups born to ZIKV-R103451- infected Atg6 ${ }^{+/-}$dams showed growth impairment. Despite the use of anti-IFNAR1 mAb, viral replication was limited in our immunocompetent mice, which could account for the rare vertical transmission of virion to embryos and pups. Viral proteins, on the contrary, were observable in post mortem brain tissues. Autophagy is a common pathway involved in regulating the replication of ZIKV 
as well as other viral-infections in cells of the central nervous system [13, 15, 26-30]. Others have shown restricted ZIKV infection in placenta, with reduced ZIKV-mediated placental damage and reduced adverse fetal outcomes using an autophagy deficient animal model lacking the Atg16L gene [31]. The contrasting data suggests that specific proteins within the autophagy pathway may be involved in ZIKV-pathogenesis.

The mechanism(s) mediating growth and brain impairments with ZIKV infection are still unclear. Our data showed a significant downregulation of several microcephaly related genes including MCPH1 and ASPM in post-mortem brains of pups deficient in the Beclin1 protein, while exposure to ZIKV (irrespective of strains) led to a further decrease in the expression of MCPH1, ASPM, WDR62, and CASC5. Studies have shown that mutations in the human WDR62 resulted in microcephaly and a wide spectrum of cortical abnormalities [32-34], while a loss in the WDR62 protein function in mice causes mitotic delay, death of neuron progenitor cells, reduced brain size and dwarfism [34]. CASC5 was shown to be involved in cell cycle and kinetochore formation during metaphase with mutation in this gene was also implicated in causing microcephaly [35]. Although the exact mechanism through which Beclin1 or the autophagy pathway causes a reduction in the microcephaly-related genes is still unknown, Beclin1 and the ultraviolet irradiation resistance-associated gene (UVRAG) are involved in both autophagy and centrosome stability. Centrosome instability and impaired autophagy can be linked to ZIKV mediated microcephaly [36, 37]. A recently identified member of MCPH (MCPH18), a phosphatidylinositol 3-phosphate-binding protein functions as a scaffold protein for autophagic removal of aggregated proteins indicating the potential role of autophagy in the development of primary microcephaly [38]. Using glia derived from beclin1 $\left(\operatorname{Atg} 6^{+/}\right)$ 
deficient animals we showed higher levels of inflammatory molecules after exposure to virus and viral proteins. While we cannot conclude that inflammation may lead to abnormal brain development, others have shown that the presence of viral proteins in the CNS causes neuroinflammation, glial dysfunction, excitotoxicity, and neuronal death [15, 39]. Ongoing studies are further investigating potential mechanisms mediating growth and brain impairments with ZIKV infection.

Another enigma is related to the transmigration of ZIKV proteins across the blood brain barrier. It was recently shown that secretory autophagy or exosome pathways could be a potential mechanism in virus transfer [40], since spreading of virus and viral toxins across cells can be facilitated by extracellular vesicles, microvesicles and exosomes [41]. Exosome biogenesis and the autophagy pathway are interlinked pathway and most importantly, implications of exosome and autophagy interplay in the context of neurodegeneration has been shown by others [42-44]. While this topic is beyond the scope of our current project, experimental studies are investigating mechanisms involved in viral trafficking.

In summary, our study showed a novel autophagy-deficient mouse model of ZIKV infection and an indirect role of the protein Beclin1 and the autophagy pathway in ZIKV infection and pathology. Lack of autophagy leads to stillbirth as well as maternal and fetal death while concurrently enhances ZIKV-mediated growth retardation by regulating the expression of microcephaly-related genes. Modulating the autophagy pathway can be employed as a therapeutic approach for ZIKV infection; however, it requires further understanding of the exact molecular mechanism and exact cellular target of the ZIKVinfection and the associated adverse outcome during pregnancy. 


\section{References}

[1] de Araujo TVB, Rodrigues LC, de Alencar Ximenes RA, de Barros MirandaFilho D, Montarroyos UR, de Melo APL, et al. Association between Zika virus infection and microcephaly in Brazil, January to May, 2016: preliminary report of a case-control study. Lancet Infect Dis. 2016;16(12):1356-63.

[2] Jaenisch T, Rosenberger KD, Brito C, Brady O, Brasil P, Marques ET. Risk of microcephaly after Zika virus infection in Brazil, 2015 to 2016. Bulletin of the World Health Organization. 2017;95(3):191-8.

[3] Parra B, Lizarazo J, Jimenez-Arango JA, Zea-Vera AF, Gonzalez-Manrique G, Vargas J, et al. Guillain-Barre Syndrome Associated with Zika Virus Infection in Colombia. N Engl J Med. 2016;375(16):1513-23.

[4] D'Ortenzio E, Matheron S, Yazdanpanah Y, de Lamballerie X, Hubert B, Piorkowski G, et al. Evidence of Sexual Transmission of Zika Virus. N Engl J Med. 2016;374(22):2195-8.

[5] Foy BD, Kobylinski KC, Chilson Foy JL, Blitvich BJ, Travassos da Rosa A, Haddow AD, et al. Probable non-vector-borne transmission of Zika virus, Colorado, USA. Emerg Infect Dis. 2011;17(5):880-2.

[6] McCarthy M. First US case of Zika virus infection is identified in Texas. BMJ. 2016;352:i212.

[7] Musso D, Roche C, Robin E, Nhan T, Teissier A, Cao-Lormeau VM. Potential sexual transmission of Zika virus. Emerg Infect Dis. 2015;21(2):359-61.

[8] Brasil P, Pereira JP, Jr., Moreira ME, Ribeiro Nogueira RM, Damasceno L, Wakimoto M, et al. Zika Virus Infection in Pregnant Women in Rio de Janeiro. N Engl J Med. 2016;375(24):2321-34.

[9] Driggers RW, Ho CY, Korhonen EM, Kuivanen S, Jaaskelainen AJ, Smura T, et al. Zika Virus Infection with Prolonged Maternal Viremia and Fetal Brain Abnormalities. N Engl J Med. 2016;374(22):2142-51.

[10] Petersen LR, Jamieson DJ, Powers AM, Honein MA. Zika Virus. N Engl J Med. 2016;374(16):1552-63.

[11] Mlakar J, Korva M, Tul N, Popovic M, Poljsak-Prijatelj M, Mraz J, et al. Zika Virus Associated with Microcephaly. N Engl J Med. 2016;374(10):951-8. 
[12] Rasmussen SA, Jamieson DJ, Honein MA, Petersen LR. Zika Virus and Birth Defects--Reviewing the Evidence for Causality. N Engl J Med. 2016;374(20):1981-7.

[13] Dick GW, Kitchen SF, Haddow AJ. Zika virus. I. Isolations and serological specificity. Trans R Soc Trop Med Hyg. 1952;46(5):509-20.

[14] Barbelanne M, Tsang WY. Molecular and cellular basis of autosomal recessive primary microcephaly. Biomed Res Int. 2014;2014:547986.

[15] Faheem M, Naseer MI, Rasool M, Chaudhary AG, Kumosani TA, Ilyas AM, et al. Molecular genetics of human primary microcephaly: an overview. BMC Med Genomics. 2015;8 Suppl 1:S4.

[16] Gilmore EC, Walsh CA. Genetic causes of microcephaly and lessons for neuronal development. Wiley Interdiscip Rev Dev Biol. 2013;2(4):461-78.

[17] Li C, Xu D, Ye Q, Hong S, Jiang Y, Liu X, et al. Zika Virus Disrupts Neural Progenitor Development and Leads to Microcephaly in Mice. Cell Stem Cell. 2016;19(1):120-6.

[18] Tang H, Hammack C, Ogden SC, Wen Z, Qian X, Li Y, et al. Zika Virus Infects Human Cortical Neural Progenitors and Attenuates Their Growth. Cell Stem Cell. 2016;18(5):587-90.

[19] Zhang F, Hammack C, Ogden SC, Cheng Y, Lee EM, Wen Z, et al. Molecular signatures associated with ZIKV exposure in human cortical neural progenitors. Nucleic Acids Res. 2016;44(18):8610-20.

[20] Ojha CR, Rodriguez M, Karuppan MKM, Lapierre J, Kashanchi F, El-Hage N. Toll-like receptor 3 regulates Zika virus infection and associated host inflammatory response in primary human astrocytes. PLOS ONE. 2019;14(2):e0208543.

[21] Liang Q, Luo Z, Zeng J, Chen W, Foo S-S, Lee S-A, et al. Zika Virus NS4A and NS4B Proteins Deregulate Akt-mTOR Signaling in Human Fetal Neural Stem Cells to Inhibit Neurogenesis and Induce Autophagy. Cell Stem Cell. 19(5):66371.

[22] Hamel R, Dejarnac O, Wichit S, Ekchariyawat P, Neyret A, Luplertlop N, et al. Biology of Zika Virus Infection in Human Skin Cells. Journal of virology. 2015;89:8880-96. 
[23] Klionsky DJ, Baehrecke EH, Brumell JH, Chu CT, Codogno P, Cuervo AM, et al. A comprehensive glossary of autophagy-related molecules and processes (2nd edition). Autophagy. 2011;7(11):1273-94.

[24] Kiriyama Y, Nochi H. The Function of Autophagy in Neurodegenerative Diseases. Int J Mol Sci. 2015;16(11):26797-812.

[25] Rodriguez M, Kaushik A, Lapierre J, Dever SM, El-Hage N, Nair M. ElectroMagnetic Nano-Particle Bound Beclin1 siRNA Crosses the Blood-Brain Barrier to Attenuate the Inflammatory Effects of HIV-1 Infection in Vitro. Journal of neuroimmune pharmacology : the official journal of the Society on NeuroImmune Pharmacology. 2017;12(1):120-32.

[26] Lapierre J, Rodriguez M, Ojha CR, El-Hage N. Critical Role of Beclin1 in HIV Tat and Morphine-Induced Inflammation and Calcium Release in Glial Cells from Autophagy Deficient Mouse. Journal of neuroimmune pharmacology : the official journal of the Society on NeuroImmune Pharmacology. 2018.

[27] Gurwell JA, Nath A, Sun Q, Zhang J, Martin KM, Chen Y, et al. Synergistic neurotoxicity of opioids and human immunodeficiency virus-1 Tat protein in striatal neurons in vitro. Neuroscience. 2001;102(3):555-63.

[28] Boland B, Kumar A, Lee S, Platt FM, Wegiel J, Yu WH, et al. Autophagy induction and autophagosome clearance in neurons: relationship to autophagic pathology in Alzheimer's disease. J Neurosci. 2008;28(27):6926-37.

[29] Chiramel AI, Best SM. Role of autophagy in Zika virus infection and pathogenesis. Virus Res. 2018;254:34-40.

[30] Rodriguez M, Lapierre J, Ojha CR, Estrada-Bueno H, Dever SM, Gewirtz DA, et al. Importance of Autophagy in Mediating Human Immunodeficiency Virus (HIV) and Morphine-Induced Metabolic Dysfunction and Inflammation in Human Astrocytes. Viruses. 2017;9(8).

[31] Houtman J, Freitag K, Gimber N, Schmoranzer J, Heppner FL, Jendrach M. Beclin1-driven autophagy modulates the inflammatory response of microglia via NLRP3. The EMBO Journal. 2019:e99430.

[32] Dever SM, Rodriguez M, Lapierre J, Costin BN, El-Hage N. Differing roles of autophagy in HIV-associated neurocognitive impairment and encephalitis with implications for morphine co-exposure. Frontiers in Microbiology. 2015;6(653).

[33] Wu K-Y, Zuo G-L, Li X-F, Ye Q, Deng Y-Q, Huang X-Y, et al. Vertical transmission of Zika virus targeting the radial glial cells affects cortex development of offspring mice. Cell Research. 2016;26:645. 
[34] Li C, Xu D, Ye Q, Hong S, Jiang Y, Liu X, et al. Zika Virus Disrupts Neural Progenitor Development and Leads to Microcephaly in Mice. Cell Stem Cell. 2016;19(1):120-6.

[35] Schweighardt B, Atwood WJ. Glial cells as targets of viral infection in the human central nervous system. Progress in Brain Research. 132: Elsevier; 2001. p. 72135 .

[36] Furr SR, Marriott I. Viral CNS infections: role of glial pattern recognition receptors in neuroinflammation. Frontiers in Microbiology. 2012;3:201.

[37] Puerta-Guardo H, Glasner DR, Harris E. Dengue Virus NS1 Disrupts the Endothelial Glycocalyx, Leading to Hyperpermeability. PLOS Pathogens. 2016;12(7):e1005738.

[38] Morrison TE, Diamond MS. Animal Models of Zika Virus Infection, Pathogenesis, and Immunity. J Virol. 2017;91(8).

[39] Pawitwar SS, Dhar S, Tiwari S, Ojha CR, Lapierre J, Martins K, et al. Overview on the Current Status of Zika Virus Pathogenesis and Animal Related Research. J Neuroimmune Pharmacol. 2017;12(3):371-88.

[40] Navratil N. A129 and AG129 Mice Are Valuable for Evaluating Zika Virus Vaccines and Treatments: Marshall bioresources; 2016. Available from: https://www.marshallbio.com/a129-and-ag129-mice-are-valuable-for-evaluatingzika-virus-vaccines-and-treatments.

[41] Smith DR, Hollidge B, Daye S, Zeng X, Blancett C, Kuszpit K, et al. Neuropathogenesis of Zika Virus in a Highly Susceptible Immunocompetent Mouse Model after Antibody Blockade of Type I Interferon. PLoS Negl Trop Dis. 2017;11(1).

[42] Lazear HM, Govero J, Smith AM, Platt DJ, Fernandez E, Miner JJ, et al. A Mouse Model of Zika Virus Pathogenesis. Cell Host Microbe. 2016;19(5):720-30.

[43] Yockey LJ, Varela L, Rakib T, Khoury-Hanold W, Fink SL, Stutz B, et al. Vaginal Exposure to Zika Virus during Pregnancy Leads to Fetal Brain Infection. Cell. 2016;166(5):1247-56 e4.

[44] Miner JJ, Cao B, Govero J, Smith AM, Fernandez E, Cabrera OH, et al. Zika Virus Infection during Pregnancy in Mice Causes Placental Damage and Fetal Demise. Cell. 2016;165(5):1081-91. 
[45] Szaba FM, Tighe M, Kummer LW, Lanzer KG, Ward JM, Lanthier P, et al. Zika virus infection in immunocompetent pregnant mice causes fetal damage and placental pathology in the absence of fetal infection. PLOS Pathogens. 2018;14(4):e1006994.

[46] Cao B, Parnell LA, Diamond MS, Mysorekar IU. Inhibition of autophagy limits vertical transmission of Zika virus in pregnant mice. The Journal of Experimental Medicine. 2017.

[47] Carneiro LA, Travassos LH. Autophagy and viral diseases transmitted by Aedes aegypti and Aedes albopictus. Microbes Infect. 2016;18(3):169-71.

[48] Chahar HS, Bao X, Casola A. Exosomes and Their Role in the Life Cycle and Pathogenesis of RNA Viruses. Viruses. 2015;7(6):3204-25.

[49] Zhang Z-W, Li Z-L, Yuan S. The Role of Secretory Autophagy in Zika Virus Transfer through the Placental Barrier. Frontiers in Cellular and Infection Microbiology. 2016;6:206.

[50] Faheem M, Naseer MI, Rasool M, Chaudhary AG, Kumosani TA, Ilyas AM, et al. Molecular genetics of human primary microcephaly: an overview. BMC Medical Genomics. 2015;8(Suppl 1):S4-S.

[51] Okamoto N, Kohmoto T, Naruto T, Masuda K, Imoto I. Primary microcephaly caused by novel compound heterozygous mutations in ASPM. Human Genome Variation. 2018;5:18015.

[52] Nicholas AK, Khurshid M, Désir J, Carvalho OP, Cox JJ, Thornton G, et al. WDR62 is associated with the spindle pole and is mutated in human microcephaly. Nature genetics. 2010;42(11):1010-4.

[53] Yu TW, Mochida GH, Tischfield DJ, Sgaier SK, Flores-Sarnat L, Sergi CM, et al. Mutations in WDR62, encoding a centrosome-associated protein, cause microcephaly with simplified gyri and abnormal cortical architecture. Nature genetics. 2010;42(11):1015-20.

[54] Chen J-F, Zhang Y, Wilde J, Hansen KC, Lai F, Niswander L. Microcephaly disease gene Wdr62 regulates mitotic progression of embryonic neural stem cells and brain size. Nature Communications. 2014;5:3885.

[55] Szczepanski S, Hussain MS, Sur I, Altmüller J, Thiele H, Abdullah U, et al. A novel homozygous splicing mutation of CASC 5 causes primary microcephaly in a large Pakistani family. Human Genetics. 2016;135(2):157-70. 
[56] Stiles J, Jernigan TL. The Basics of Brain Development. Neuropsychology Review. 2010;20(4):327-48.

[57] Ponti G, Peretto P, Bonfanti L. Genesis of Neuronal and Glial Progenitors in the Cerebellar Cortex of Peripuberal and Adult Rabbits. PLoS ONE. 2008;3(6):e2366.

[58] Gilmore EC, Walsh CA. Genetic Causes of Microcephaly and Lessons for Neuronal Development. Wiley interdisciplinary reviews Developmental biology. 2013;2(4):461-78.

[59] Mathew R, Kongara S, Beaudoin B, Karp CM, Bray K, Degenhardt K, et al. Autophagy suppresses tumor progression by limiting chromosomal instability. Genes \& Development. 2007;21(11):1367-81.

[60] Zhao Z, Oh S, Li D, Ni D, Pirooz Sara D, Lee J-H, et al. A Dual Role for UVRAG in Maintaining Chromosomal Stability Independent of Autophagy. Developmental Cell. 2012;22(5):1001-16.

[61] Kadir R, Harel T, Markus B, Perez Y, Bakhrat A, Cohen I, et al. ALFYControlled DVL3 Autophagy Regulates Wnt Signaling, Determining Human Brain Size. PLOS Genetics. 2016;12(3):e1005919. 


\section{CHAPTER 7. CONCLUSION AND RECOMMENDATIONS}

Despite the myriad of studies focusing on the molecular mechanism of ZIKV infection, there are many questions that still remain unanswered. The exact mechanisms by which the virus enters host cells and mediates brain anomalies in fetus and neurological complications in adults are still not conclusive. We aimed at exploring the molecular mechanism of ZIKV infection and associated neuropathogensis. In the line with other evidences our study shows that ZIKV uses Axl receptor for its cellular entry with Tyro3 playing no significant role in ZIKV infection in the glial cells. Besides viral entry, Axl receptor is involved in ZIKV replication inside the glial cells. The data suggest that targeting Axl receptor can attenuate ZIKV infection by blocking the cellular entry of the virus and limiting the replication. However, inhibition of Axl in turn may exacerbate the ZIKV-induced inflammatory responses. Once ZIKV enters the host, TLR3 senses the virus and get activated. Activation of TLR3 leads to an antiviral response, however, exaggerated immune response mediated by hyper-activation of TLR3 may be associated with ZIKV pathogenesis. Therefore, TLR3 could be a target for therapeutic approach [1].

Upon activation of TLR receptors, a burst of pro-inflammatory cytokines is released, which then propagates inflammation by stimulating various cytokine receptors, including type I interferon receptors (IFNAR). Though the inflammatory response is a part of body immune mechanism, it has to be controlled in terms of time and magnitude to prevent from the unwanted, detrimental or autoimmune responses. The immune system employs multiple strategies to turn off or limit the inflammation. One of the crucial mechanism is the TAM signaling pathways. Activation of TAM receptors by IFNARs via JAK/STAT pathway dampen inflammatory responses downstream of TLR and cytokine receptors by 
increasing the expression of the suppressor of cytokine signaling proteins (SOCS). Therefore, TAM signaling pathways function as mechanisms of negative feedback in inflammation. Whereas, TAM receptors themselves are under the control of TLR signaling pathways which assures that the initiation of TAM pathways only occurs at the end stages of the immune responses, allowing efficient inflammatory responses to occur while preventing chronic inflammation and concomitant tissue damage [1].

ZIKV activates the autophagy pathway however mediating autophagy pathway by activation and inhibition of various steps in the pathway might not necessarily inhibit ZIKV infection. Autophagy protein Beclin1 plays a protective role by suppressing ZIKV-induced inflammation, controlling the microcephaly associated genes expression in in-utero ZIKV exposed pups and limiting the congenital ZIKV syndromes and neurodevelopmental consequences associated with ZIKV. Both the autophagy and the TLR3 pathways are important mediators of the innate immune response that are activated by ZIKV. The different receptors in the TLR pathways are the initiators of both innate and adaptive immune response, while the autophagy pathway clears intracellular pathogens and facilitates antigen presentation. Since, TLR signaling pathway is reported to induce the autophagy pathway [2-5], there may be a possible connection between ZIKV induced TLR3 pathway and autophagy pathway. MyD88 and TRIF, two adaptor proteins involved in the TLR signaling pathway, can interact with the autophagy protein, Beclin1 and this interaction inhibit binding between Beclin1 and the anti-apoptotic protein, B-cell lymphoma 2 (Bcl-2). Dissociation of Beclin1 from Bcl-2 by MyD88 and TRIF induces autophagy [5], while autophagy processing of viral RNA activates TLR signaling pathway. Our data further indicate that ZIKV associated autophagy induction is at least partially 
mediated via activation of TLR signaling. ZIKV can also induce ER stress related pathways depending on the viral strains. In summary, ZIKV can modulate vital cellular mechanisms including the TLR, UPR, and autophagy pathways, with cumulative consequences of cellular destruction and clinical manifestations.

We further studied the role of autophagy pathway, specifically the Beclin1, in development of neuropathology in in-utero ZIKV-exposed fetus by using ZIKV-infected timed-pregnant wild-type C57BL/6J and Beclin1 deficient (Atg6 $6^{+/}$) mice. We showed that autophagy pathway or the Beclin1 protein plays a protective role in both growth and embryonic brain development in the fetus. Beclin1 deficiency contributes to the ZIKVinduced reduction in several microcephalic genes in the brain leading to the overt congenital malformations and growth impairment in the offspring. Finally, our data indicate that ZIKV proteins transmigrated from the placenta to the fetus probably via the secretory autophagy pathway may be the main causal factors for the neurodevelopmental delay detected in pups born from ZIKV-infected dams.

Despite our effort to explore in detail mechanism of ZIKV infection and associated neuropathogensis, there are some limitations in our studies. Because of lack of time and resources, we couldn't confirm the role of TLR3 and Axl using in-vivo animal model. Similarly, we are lacking the data for protein expression of microcephaly related genes. We are still examining the potential role of secretory autophagy or exosome in the transmigration of ZIKV proteins or genome.

Development of therapeutics requires precise understanding of the biology and pathogenesis of the virus. The major challenge in ZIKV research is to directly correlate viral infection with viral induced neurodevelopmental disorders in an immunocompetent 
animal model. Exact cellular and molecular mechanisms of ZIKV pathogenesis may not be reflective in immunocompromised mice. Therefore, development of a more appropriate animal model system is essential not only for understanding the biology of infection, but also for determining the targets for vaccines and therapies. While studying the antiviral drugs, safety in pregnant woman should also be evaluated because common antivirals and nucleoside analogs are reported to be unsafe during pregnancy, therefore alternative therapeutic approaches that target cell signaling pathways can be explored. Based on some of the current findings, more investigations are necessary to exploit TLR3 and the autophagy pathways as potential therapeutic targets. Since neurodevelopmental disorders, neuroinflammation and neurodegenerative consequences are major public health threats concerning ZIKV infection, drug delivery to central nervous system by employing targeted drug delivery approaches such as nanoparticles, nanogels, liposomes or implants need to be studied after the identification of suitable targets.

\section{References}

[1] Dang J, Tiwari SK, Lichinchi G, Qin Y, Patil VS, Eroshkin AM, et al. Zika Virus Depletes Neural Progenitors in Human Cerebral Organoids through Activation of the Innate Immune Receptor TLR3. Cell stem cell. 2016;19(2):258-65.

[2] Delgado MA, Elmaoued RA, Davis AS, Kyei G, Deretic V. Toll-like receptors control autophagy. EMBO J. 2008;27(7):1110-21.

[3] Daffis S, Samuel MA, Suthar MS, Jr. MG, Diamond MS. Toll-Like Receptor 3 Has a Protective Role against West Nile Virus Infection. Journal of Virology. 2008 .

[4] Tabeta K, Georgel P, Janssen E, Du X, Hoebe K, Crozat K, et al. Toll-like receptors 9 and 3 as essential components of innate immune defense against mouse cytomegalovirus infection. Proceedings of the National Academy of Sciences. 2004. 
[5] Shi C-S, Kehrl JH. MyD88 and Trif Target Beclin 1 to Trigger Autophagy in Macrophages. Journal of Biological Chemistry. 2008;283:33175-82. 
VITA

\author{
CHET RAJ OJHA \\ Florida International University \\ Department of Immunology and Nanomedicine \\ 11200 SW $8^{\text {th }}$ ST, Miami, FL, 33199, United States
}

2009-2012

Tribhuvan University, Central department of microbiology,

Kathmandu, Nepal

MS, Medical microbiology

2015-2019

\author{
Florida International University, \\ College of Medicine, \\ $\mathrm{PhD}$, Biomedical Sciences, \\ Presidential award \\ Graduate research assistant \\ Dissertation year fellowship
}

\title{
JOURNAL PUBLICATIONS AND PRESENTATIONS
}

Chet Raj Ojha, Myosotys Rodriguez, Mohan Kumar Muthu Karupan, Jessica Lapierre, Fatah Kashanchi, and Nazira El-Hage. Toll-like receptor 3 regulates Zika virus infection and associated host inflammatory response in primary human astrocytes. PLoS ONE 14(2): e0208543.

Myosotys Rodriguez, Jessica Lapierre, Chet Raj Ojha, Shashank S. Pawitwar, Mohan Kumar Muthu Karupan, Fatah Kashanchi, Nazira El-Hage. Morphine counteracts the antiviral effect of antiretroviral drugs and causes upregulation of p62/SQSTM1 and histone modifying enzymes in HIV-infected astrocytes. Journal of Neurovirology, 201.

Chet Raj Ojha, Myosotys Rodriguez, Jessica Lapierre, Mohan Kumar Muthu Karupan, Heather Branscome, Fatah Kashanchi, and Nazira El-Hage. Complementary mechanisms potentially involved in the pathology of Zika Virus. Front. Immunol. 2018. doi:

10.3389/fimmu.2018.02340.

Jessica Lapierre, Myosotys Rodriguez, Chet Raj Ojha, Nazira El-Hage. Critical Role of Beclin1 in HIV Tat and Morphine-Induced Inflammation and Calcium Release in Glial Cells from Autophagy Deficient Mouse. Journal of Neuroimmune Pharmacology 05/2018; DOI:10.1007/s11481-018-9788-3

Myosotys Rodriguez, Jessica Lapierre, Chet Raj Ojha, Hary Estrada-Bueno, Seth M.

Dever, David A. Gewirtz, Fatah Kashanchi, Nazira El-Hage. Importance of Autophagy in Mediating Human Immunodeficiency Virus (HIV) and Morphine-Induced Metabolic 
Dysfunction and Inflammation in Human Astrocytes. Viruses 07/2017; 9(8):201, DOI:10.3390/v9080201

Chet Raj Ojha, Jessica Lapierre, Myosotys Rodriguez, Seth M. Dever, Mohammad Asad Zadeh, Catherine DeMarino, Michelle L. Pleet, Fatah Kashanchi, Nazira El-Hage. Interplay between Autophagy, Exosomes and HIV-1 Associated Neurological Disorders: New Insights for Diagnosis and Therapeutic Applications. Viruses 07/2017; 9(7):176., DOI: $10.3390 / \mathrm{v} 9070176$

Myosotys Rodriguez, Jessica Lapierre, Chet Raj Ojha, Ajeet Kaushik, Elena Batrakova, Fatah Kashanchi, Seth M. Dever, Madhavan Nair, Nazira El-Hage. Intranasal drug delivery of small interfering RNA targeting Beclin1 encapsulated with polyethylenimine (PEI) in mouse brain to achieve HIV attenuation. Scientific Reports 05/2017; 7(11):1862., DOI:10.1038/s41598-017-01819-9

Shashank S. Pawitwar, Supurna Dhar, Sneham Tiwari, Chet Raj Ojha, Jessica Lapierre, Kyle Martins, Alexandra Rodzinski, Tiyash Parira, Iru Paudel, Jiaojiao Li, Rajib Kumar Dutta, Monica R. Silva, Ajeet Kaushik, Nazira El-Hage. Overview on the Current Status of Zika Virus Pathogenesis and Animal Related Research. Journal of Neuroimmune Pharmacology 04/2017; 12(6304)., DOI:10.1007/s11481-017-9743-8

Chet Raj Ojha, Myosotys Rodriguez, Seth M. Dever, Rita Mukhopadhyay, Nazira ElHage. Mammalian microRNA: an important modulator of host-pathogen interactions in human viral infections. Journal of Biomedical Science 12/2016; 23(1)., DOI:10.1186/s12929-016-0292-X

Chet Raj Ojha (2017).Drug-drug interactive effect of morphine on cART leads to imbalances in autophagy pathway in HIV-infected astrocytes. Paper presented at 23rd Scientific Conference of Society on neuroimmune pharmacology.

Chet Raj Ojha (2017). Neuropathogenesis mediated by Zika virus and role of autophagy". Oral presentation at HWCOM annual research symposium.

Chet Raj Ojha (2018). Understanding the Role of Autophagy as a mechanism of neuroinflammation in Zika virus Infected glial cells. Poster presentation at Keystone symposia on molecular and cellular biology, selective autophagy meeting.

Chet Raj Ojha (2017). "Neuropathogenesis mediated by Zika virus and the role of autophagy". Poster presentation at American society of virology (ASV) $36^{\text {th }}$ Annual meeting. 